\begin{tabular}{|c|c|}
\hline $\begin{array}{l}\overline{\overline{\bar{n}}} \\
\text { AOMOMA }\end{array}$ & $\begin{array}{l}\text { DiPARTIMENTO DI INFORMATICA E AUtOMAZIONE } \\
\text { Via della Vasca Navale, } 79 \\
\text { 00146 Roma, Italy }\end{array}$ \\
\hline
\end{tabular}

\title{
On the Queue Number of Planar Graphs
}

Giuseppe Di Battista ${ }^{1}$, Fabrizio Frati ${ }^{1}$, JÁnos PaCH $^{2}$

RT-DIA-169-2010

April 2010

(1) Università Roma Tre, Italy

(2) EPFL Lausanne, Switzerland

Work partially supported by the Italian Ministry of Research, Grant number RBIP06BZW8, project FIRB "Advanced tracking system in intermodal freight transportation". 


\begin{abstract}
We prove that planar graphs have $O\left(\log ^{4} n\right)$ queue number, thus improving upon the previous $O(\sqrt{n})$ upper bound. Consequently, planar graphs admit 3D straight-line crossingfree grid drawings in $O\left(n \log ^{c} n\right)$ volume, for some constant $c$, thus improving upon the previous $O\left(n^{3 / 2}\right)$ upper bound.
\end{abstract}




\section{Introduction and Overview}

A linear layout of a graph is a total ordering of its vertices and a partition of its edges such that all the elements of the partition enforce some specific property.

Linear layouts play an important role in Graph Theory and their study goes back to 1973, when Ollmann [27] introduced the concept of book embedding (later also called stack layout) and book thickness (later also called stack number, fixed outer-thickness, and, most successfully, page number) of a graph. A book embedding on $k$ pages of a graph $G(V, E)$ is a linear layout of $G$ in which the partition of $E$ consists of $k$ sets $E_{1}, E_{2}, \ldots, E_{k}$, called pages, such that no two edges in the same page cross (edges $(u, v)$ and $(w, z)$ cross if $u<w<v<z$ or $w<u<z<v)$, and the page number is the minimum $k$ such that $G$ has a book embedding on $k$ pages. The literature is rich of combinatorial and algorithmic contributions on the page number of various classes of graphs (see, e.g., [4, 18, 15, 19, 25, 26, 13, 12, 17, 16]). A famous result of Yannakakis [33] states that a planar graph has page number at most four.

Queue layout and queue number are the dual concepts of book embedding and page number, respectively. A queue layout on $k$ queues of a graph $G(V, E)$ is a linear layout of $G$ in which the partition of $E$ consists of $k$ sets $E_{1}, E_{2}, \ldots, E_{k}$, called queues, such that no two edges in the same queue nest (edges $(u, v)$ and $(w, z)$ nest if $u<w<z<v$ or $w<u<v<z)$, and the queue number is the minimum $k$ such that $G$ has a queue layout on $k$ queues. Queue layouts were introduced by Heath, Leighton, and Rosenberg [20, 24], motivated by applications, e.g., in parallel process scheduling [1], matrix-computations [28], and sorting permutations and networks [29, 32].

Computing the queue number of a graph is $\mathcal{N} \mathcal{P}$-complete. Namely, it is known that deciding if a graph has queue number 1 is $\mathcal{N} \mathcal{P}$-complete [24]. However, from a combinatorial point of view, a large number of bounds are known on the queue number of several graph classes. For example, graphs with $m$ edges, graphs with tree-width $w$, graphs with tree-width $w$ and degree $\Delta$, graphs with path-width $p$, graphs with bandwidth $b$, and graphs with track number $t$ have queue number at most $e \sqrt{m}$ [9], at most $3^{w} \cdot 6^{\left(4^{w}-3 w-1\right) / 9}-1[8]$, at most $36 \Delta w[8]$, at most $p[8]$, at most $\lceil b / 2\rceil[24]$, and at most $t-1$ [8], respectively. Queue layouts of directed graphs [23, 22] and posets [21] have also been studied.

As in many graph problems, a special attention has been devoted to planar graphs and their subclasses. For example, trees have queue number 1 [24], outerplanar graphs have queue number 2 [20], and series-parallel graphs have queue number 3 [30]. However, for general planar graphs the best known upper bound for the queue number is $O(\sqrt{n}$ ) (a consequence of the results on graphs with $O(n)$ edges $[24,31,9]$ ), while no super-constant lower bound is known. Heath et al. [20, 24] conjectured that planar graphs have $O(1)$ queue number. Pemmaraju [28] conjectured that a certain class of planar graphs, namely planar 3-trees, have $\Omega(\log n)$ queue number. However, Dujmović et al. [8] disproved such a conjecture by proving that graphs of constant tree-width, and hence also planar 3trees, have constant queue number. Observe that the problem of determining the queue number of planar graphs is cited into several papers and collections of open problems (see, e.g., $[20,24,6,3,7,11,8])$.

In this paper, we prove that the queue number of planar graphs is $O\left(\log ^{4} n\right)$. The proof is constructive and is based on a polynomial-time algorithm that computes a queue layout with such a queue number. The result is based on several new combinatorial and 
algorithmic tools.

First (Sect. 2), we introduce level-2-connected graphs, that are plane graphs in which each outerplanar level induces a set of disjoint 2-connected graphs. We show that every planar graph has a subdivision with one vertex per edge that is a level-2-connected graph. Such a result, together with a result of Dujmović and Wood [11] stating that the queue number of a graph $G$ is at most the square of the queue number of a subdivision of $G$ with one vertex per edge, allows us to study the queue number of level-2-connected graphs in order to determine bounds on the queue number of general planar graphs.

Second (Sect. 3), we introduce floored graphs, that are plane graphs where vertices are partitioned into sets $V_{1}, V_{2}, \ldots, V_{k}$ such that some strong topological properties on the subgraph induced by each $V_{i}$ and on the connectivity among such subgraphs are satisfied. We prove that every level-2-connected graph admits a partition of its vertex set resulting into a floored graph. Floored graphs are then related to the outerplanar levels of a level2-connected graph. Such levels form a tree hierarchy that can be thought as having one node for each connected component of an outerplanar level and an edge $(u, v)$ if the graph corresponding to $v$ lies inside the graph corresponding to $u$. Floored graphs are used to explore such hierarchy one path at a time. Moreover, we prove the existence in any floored graph $G$ of a simple subgraph (a path plus few edges) that decomposes $G$ into two smaller floored graphs $G^{\prime}$ and $G^{\prime \prime}$.

Third (Sect. 4), we show an algorithm that constructs a queue layout of a floored graph $G$ in which the different sets of the partition are in consecutive sub-sequences of the total vertex ordering of $G$. The algorithm is recursive and at each step uses the mentioned decomposition of a floored graph $G$ into two floored graphs $G^{\prime}$ and $G^{\prime \prime}$ several times, each time splitting the floored graph with the greatest number of vertices between the two floored graphs obtained at the previous splitting, until no obtained floored graph has more than half of the vertices of the initial floored graph. The resulting floored graphs have different vertex partitions. However, it is shown how to merge such partitions in such a way that $O\left(\log ^{2} n\right)$ queues are sufficient to accommodate all the edges of the initial $n$-vertex graph.

Then, we conclude that floored graphs have $O\left(\log ^{2} n\right)$ queue number, hence level-2connected graphs have $O\left(\log ^{2} n\right)$ queue number, thus planar graphs have $O\left(\log ^{4} n\right)$ queue number.

Our result sheds new light on one of the most studied Graph Drawing problems (see, e.g., $[5,14,3,10,8,6])$ : Given an $n$-vertex planar graph which is the volume required to draw it in $3 \mathrm{D}$, representing edges with straight-line segments that cross only at common endpoints? The previously best known upper bound [10] was $O\left(n^{1.5}\right)$ volume. We prove that planar graphs have 3D straight-line crossing-free drawings in $O\left(n \log ^{c} n\right)$ volume, for some constant $c$. Such a result comes from our new bound on the queue number of planar graphs and from results by Dujmović, Morin, and Wood [8] relating the queue number of a graph to its track number and to the volume requirements of its 3D straight-line crossing-free drawings.

\section{Preliminaries}

A planar drawing of a graph is a mapping of each vertex to a distinct point of the plane and of each edge to a Jordan curve between its endpoints such that no two edges 
intersect except, possibly, at common endpoints. A planar drawing of a graph determines a circular ordering of the edges incident to each vertex. Two drawings of the same graph are equivalent if they determine the same circular ordering around each vertex. A planar embedding is an equivalence class of planar drawings. A planar drawing partitions the plane into topologically connected regions, called faces. The unbounded face is the outer face. A graph together with a planar embedding and a choice for its outer face is called plane graph. A plane graph is maximal when all its faces are triangles. A plane graph is internally-triangulated when all its internal faces are triangles. An outerplane graph is a plane graph such that all its vertices are on the outer face.

A graph $G^{\prime}\left(V^{\prime}, E^{\prime}\right)$ is a subgraph of a graph $G(V, E)$ if $V^{\prime} \subseteq V$ and $E^{\prime} \subseteq E$. A subgraph is induced by $V^{\prime}$ if, for every edge $(u, v) \in E$ such that $u, v \in V^{\prime},(u, v) \in E^{\prime}$. The subgraph induced by $V^{\prime} \subseteq V$ is denoted by $G\left[V^{\prime}\right]$. A graph is connected if every pair of vertices is connected by a path. A $k$-connected graph $G$ is such that removing any $k-1$ vertices leaves $G$ connected. A vertex whose removal disconnects the graph is a cut-vertex. A $k$-subdivision of a graph $G$ is a graph obtained by replacing each edge of $G$ with a path having at most $2+k$ vertices. A chord of a cycle $C$ is an edge connecting two non-consecutive vertices of $C$. A chord of a plane graph $G$ is a chord of the cycle delimiting the outer face of $G$.

The outerplanar levels (or simply levels) of a plane graph $G$ are defined as follows. Let $G_{1}=G$ and let $G_{i+1}$ be the plane graph obtained by removing from $G_{i}(i \geq 1)$ the set $V_{i}$ of vertices of the outer face of $G_{i}$ and their incident edges. Set $V_{i}$ is the $i$-th level of $G$. Observe that the first level of $G$ is the set of vertices of its outer face. Let $k$ be the maximum index such that $V_{k} \neq \emptyset$. We say that $G$ has $k$ levels. A 2-connected internallytriangulated plane graph $G$ is level-2-connected if $G_{i}$ is composed of a set of 2-connected graphs that are pairwise vertex-disjoint and that have each at least three vertices, for each $1 \leq i \leq k$. That is, $G_{i}$ has no cut-vertex and it has no connected component that is a single vertex or a single edge. Fig. 1.a shows a maximal plane graph $G$ that is not level-2-connected and Fig. 1.b shows a maximal plane graph $G^{*}$ that is level-2-connected and that contains $G$ as a 1-subdivision. We have the following:

Lemma 1 Let $G$ be an n-vertex plane graph. There exists an $O(n)$-vertex maximal plane graph $G^{*}$ such that: (i) $G^{*}$ is level-2-connected, and (ii) $G^{*}$ contains a subgraph $G^{\prime}$ such that $G^{\prime}$ is a 1-subdivision of $G$.

Proof: First, we show that it suffices to prove the statement for maximal plane graphs. Namely, suppose that the statement holds for all $n$-vertex maximal plane graphs and consider any plane graph $G$. Augment $G$ to a maximal plane graph $\bar{G}$ by adding dummy edges to $G$ and construct an $O(n)$-vertex level-2-connected graph $G^{*}$ that contains a subgraph $G^{\prime}$ such that $G^{\prime}$ is a 1 -subdivision of $\bar{G}$. It follows that $G^{*}$ contains a subgraph $G^{\prime \prime}$ such that $G^{\prime \prime}$ is a 1 -subdivision of $G$, namely $G^{\prime \prime}$ can be obtained from $G^{\prime}$ by removing the edges of $\bar{G}$ not in $G$ (observe that such edges have been possibly subdivided in $G^{\prime}$ ).

Next, we show that, given an $n$-vertex maximal plane graph $G$, an $O(n)$-vertex level2-connected maximal plane graph $G^{*}$ can be constructed containing a 1-subdivision of $G$ as a subgraph. Denote by $k$ the number of levels of $G$. The $i$-th level of $G$ is denoted by $V_{i}$. Plane graph $G^{*}$ is constructed in $k$ steps. Let $G_{1}^{*}=G$ and let $G_{i+1}^{*}$ be the graph constructed by the algorithm after step $i$, where $G^{*}=G_{k+1}^{*}$. For each $1 \leq i \leq k$, suppose that before the $i$-th step the following invariants hold: (Invariant 1 ) $G_{i}^{*}$ is a maximal 


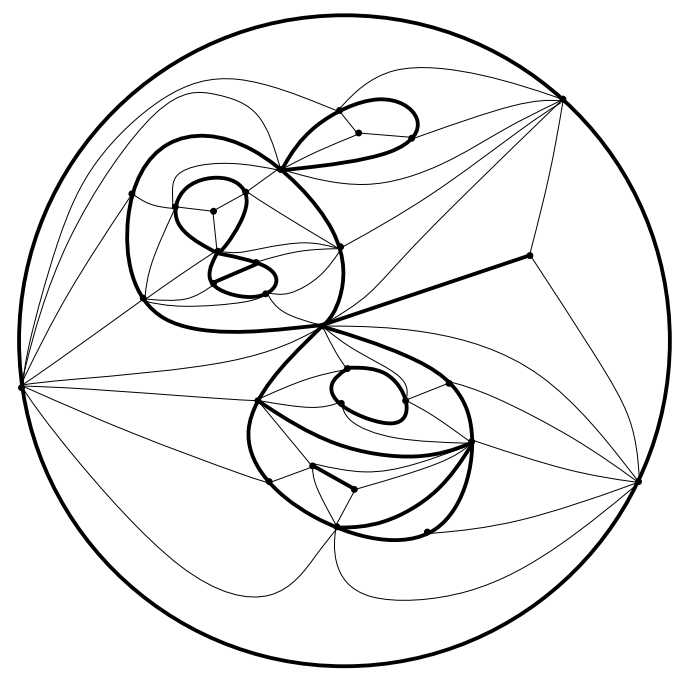

(a)

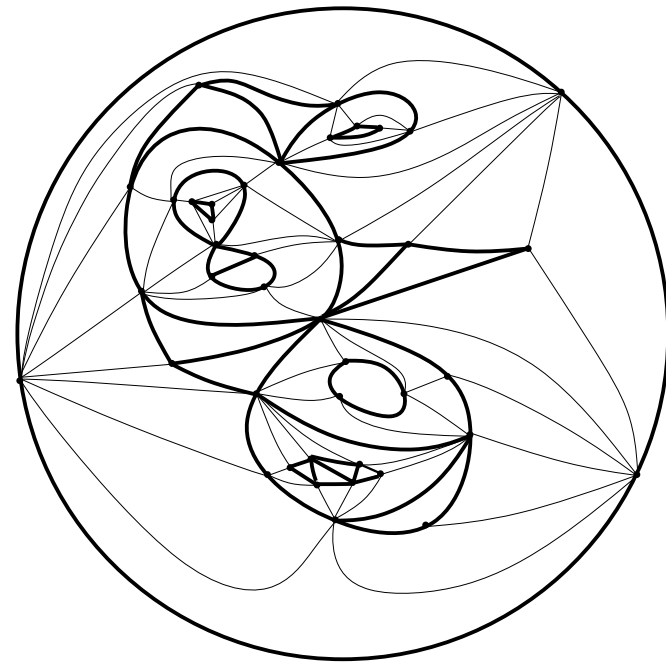

(b)

Figure 1: Two maximal plane graphs $G$ and $G^{*}$. The subgraphs induced by the levels of $G$ and $G^{*}$ are shown by thick lines. (a) $G$ is not level-2-connected. (b) $G^{*}$ is level-2-connected and contains $G$ as a 1 -subdivision.

plane graph; (Invariant 2) graph $G_{i}^{*}$ has $k$ levels $V_{i, 1}^{*}, V_{i, 2}^{*}, \ldots, V_{i, k}^{*}$, where $V_{j} \subseteq V_{i, j}^{*}$, for each $1 \leq j \leq k$; (Invariant 3) the vertices in $V_{i, j}^{*}$ induce a subgraph $O_{i, j}^{*}$ of $G_{i}^{*}$ which is composed of a set of 2-connected outerplane graphs with at least three vertices, for each $1 \leq j \leq i$. Such invariants clearly hold before step $i=1$, as $G$ is a maximal plane graph.

Step $i$ performs the following operations. For each simple cycle $C$ of $O_{i, i}^{*}$ that contains vertices and that does not contain chords in its interior, denote by $O_{i, i+1}^{*}(C)$ the subgraph of $O_{i, i+1}^{*}$ induced by the vertices of $V_{i, i+1}^{*}$ internal to $C$. Observe that such a graph is connected, as if it were not connected, then there would exists a chord internal to $C$, as $G_{i}^{*}$ is maximal, contradicting the assumptions on $C$.

- Construct an ordering $\mathcal{O}$ of the edges of $G_{i}^{*}$ connecting vertices of $C$ to vertices of $O_{i, i+1}^{*}(C)$ as follows. Suppose that $C$ is oriented clockwise. This leads us to speak of a circular ordering of the vertices of $C$. Denote by $p(v)$ the vertex preceding a vertex $v \in C$ in such an ordering of $C$. Then, start from any vertex $v_{0}(C)$ of $C$ and insert in $\mathcal{O}$ the edges of $G_{i}^{*}$ connecting $v_{0}(C)$ to vertices of $O_{i, i+1}^{*}(C)$, in their counter-clockwise order around $v_{0}(C)$ starting at the edge that follows in counterclockwise order around $v_{0}(C)$ the edge connecting $v_{0}(C)$ and $p\left(v_{0}(C)\right)$. Suppose that the edges of $G_{i}^{*}$ connecting $v_{x}(C)$ to vertices of $O_{i, i+1}^{*}$ have been inserted into $\mathcal{O}$. Denote by $v_{x+1}(C)$ the vertex following $v_{x}(C)$ on $C$. Then, append to $\mathcal{O}$ the edges of $G_{i}^{*}$ connecting $v_{x+1}(C)$ to vertices of $O_{i, i+1}^{*}$, in their counter-clockwise order around $v_{x+1}(C)$ starting at the edge that follows in counter-clockwise order around $v_{x+1}(C)$ the edge connecting $v_{x+1}(C)$ to $p\left(v_{x+1}(C)\right)$. Stop once all the edges connecting vertices of $C$ to vertices of $O_{i, i+1}^{*}(C)$ have been inserted into $\mathcal{O}$. Fig. 2.a shows a choice for $v_{0}(C)$ and the first nine edges in the resulting ordering $\mathcal{O}$.

- Consider the edges connecting vertices of $C$ to vertices of $O_{i, i+1}^{*}(C)$ in the order they appear in $\mathcal{O}$ and let $L$ be an initially empty list of vertices. If the currently considered edge $e$ is incident to a vertex $u$ such that $u$ is not a cut-vertex of $O_{i, i+1}^{*}(C)$ 


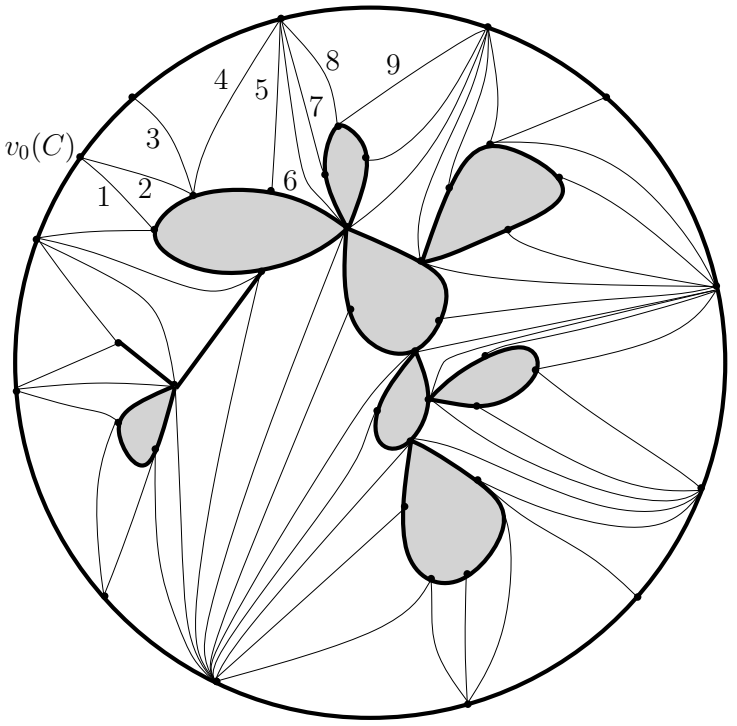

(a)

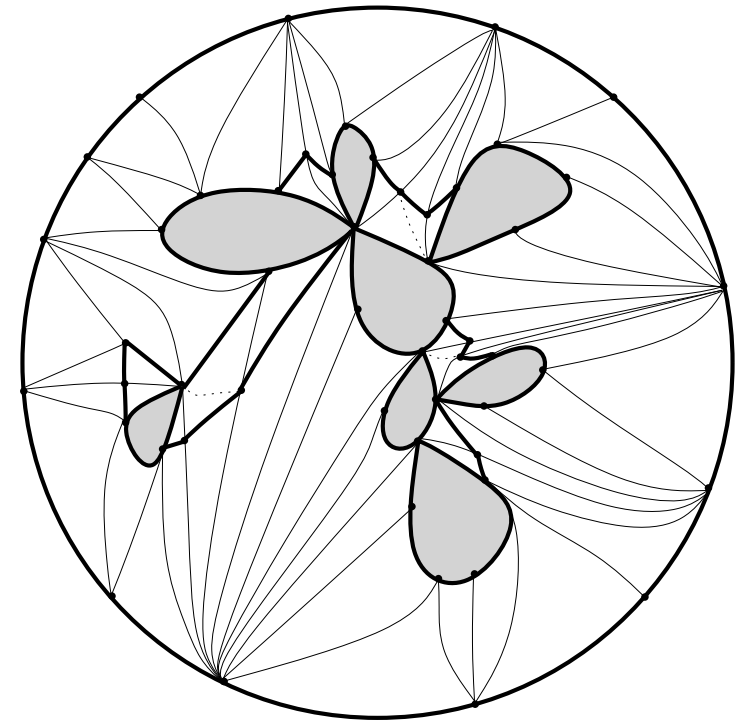

(b)

Figure 2: (a) Before step $i$ : Cycle $C$, its incident internal edges, and the outer face of graph $O_{i, i+1}^{*}(C)$ (the interior of $O_{i, i+1}^{*}(C)$ is colored gray and the edges on the outer face of $O_{i, i+1}^{*}(C)$ are thick). (b) After step $i$ : Cycle $C$, its incident internal edges, and the outer face of graph $O_{i+1, i+1}^{*}(C)$ (the edges inserted at step $i$ of the algorithm and on the outer face of $O_{i+1, i+1}^{*}(C)$ are thick and the edges inserted to augment the graph to maximal are dotted).

and $O_{i, i+1}^{*}(C)$ has at least three vertices, then append $u$ to $L$, if $u$ is not already in $L$. Otherwise (that is, the currently considered edge $e$ is incident to a vertex $u$ such that either $u$ is a cut-vertex of $O_{i, i+1}^{*}(C)$ or $O_{i, i+1}^{*}(C)$ has at most two vertices), if there is an edge incident to $u$ after $e$ in $\mathcal{O}$, then subdivide $e$ into two edges by inserting one subdivision vertex $u(e)$ in $e$ and append $u(e)$ to $L$, else (e is the last edge incident to $u$ in $\mathcal{O}$ ) append $u$ to $L$.

- Consider two consecutive vertices in $L$, where such a list is now viewed as circular. If such vertices are not the same vertex and if the edge connecting such vertices does not already exist, then insert such an edge inside the only face of $G_{i}^{*}$ that is incident to both vertices. Once all the pairs of consecutive vertices in $L$ have been considered, insert edges to triangulate the faces inside $C$ in any planar way. Fig. 2.b shows the subdivision vertices and the edges inserted in the example of Fig. 2.a.

Once such operations have been performed for each simple cycle $C$ of $O_{i, i}^{*}$ that contains vertices and that does not contain chords in its interior, denote by $G_{i+1}^{*}$ the resulting graph. We prove that $G_{i+1}^{*}$ satisfies Invariants $1-3$.

- Invariant 1. By construction, step $i$ adds no multiple edge, hence $G_{i+1}^{*}$ is simple. Each edge added between two consecutive vertices in $L$ does not cause crossings, as it is inserted inside a face incident to both such vertices; further, the edges added to triangulate the faces inside $C$ are chosen so that they do not cause crossings as well, hence $G_{i+1}^{*}$ is plane. Finally, the only triangular faces that are modified by step $i$ of the algorithm are those internal to $C$; however, edges are inserted to triangulate all such faces at the end of step $i$, hence $G_{i+1}^{*}$ is maximal. 
- Invariant 2. As every subdivision vertex and every edge inserted at step $i$ of the algorithm is internal to a cycle $C$ of $O_{i, i}^{*}$, then $V_{j} \subseteq V_{i, j}^{*}=V_{i+1, j}^{*}$, for each $1 \leq j \leq i$. To show that $V_{i+1} \subseteq V_{i+1, i+1}^{*}$, we have to prove that all the vertices of $O_{i, i+1}^{*}(C)$ are on the outer face of the graph obtained by removing the vertices of the first $i$ levels and their incident edges from $G_{i+1}^{*}$, for each subgraph $O_{i, i+1}^{*}(C)$ of $O_{i, i+1}^{*}$ induced by the vertices of $V_{i, i+1}^{*}$ internal to a simple cycle $C$ of $O_{i, i}^{*}$ that contains vertices and that does not contain chords in its interior. However, such a statement directly descends from the fact that each vertex $u$ of $O_{i, i+1}^{*}(C)$ is connected to a vertex of $C$ in $G_{i+1}^{*}$. Namely, if $u$ is not a cut-vertex of $O_{i, i+1}^{*}(C)$ and $O_{i, i+1}^{*}(C)$ has at least three vertices, then no edge incident to $u$ and incident to a vertex of $C$ is subdivided (observe that there exists at least one of such edges as $G_{i}^{*}$ is maximal); otherwise, by construction the last edge incident to $u$ is not subdivided. It remains to prove that $V_{j} \subseteq V_{i+1, j}^{*}$, for each $i+2 \leq j \leq k$. To do that it suffices to observe that all the inserted subdivision vertices belong to $V_{i+1, i+1}^{*}$; then, removing the vertices of the first $i+1$ levels and their incident edges from $G_{i+1}^{*}$, leads to the same graph as the one obtained by removing the first $i+1$ levels and their incident edges from $G_{i}^{*}$, hence $V_{j} \subseteq V_{i, j}^{*}=V_{i+1, j}^{*}$, for each $i+2 \leq j \leq k$.

- Invariant 3. As each subdivision vertex and each edge inserted at step $i$ of the algorithm is internal to a cycle $C$ of $O_{i, i}^{*}$, then the vertices in $V_{i+1, j}^{*}$ are the same vertices as in $V_{i, j}^{*}$, for each $1 \leq j \leq i$, hence they induce a subgraph $O_{i+1, j}^{*}$ of $G_{i+1}^{*}$ which is isomorphic to the subgraph $O_{i, j}^{*}$ of $G_{i}^{*}$ induced by the vertices in $V_{i, j}^{*}$, which is composed of a set of 2-connected components that are pairwise vertex-disjoint and that have each at least three vertices. To prove that the vertices in $V_{i+1, i+1}^{*}$ induce a subgraph $O_{i+1, i+1}^{*}$ of $G_{i+1}^{*}$ which is composed of a set of 2-connected components that are pairwise vertex-disjoint and that have each at least three vertices, it suffices to prove that the subgraph $O_{i+1, i+1}^{*}(C)$ of $O_{i+1, i+1}^{*}$ induced by the vertices of $V_{i+1, i+1}^{*}$ internal to $C$ is 2-connected and has at least three vertices, for each simple cycle $C$ of $O_{i, i}^{*}$ that contains vertices and that does not contain chords in its interior.

First, we prove that $O_{i+1, i+1}^{*}(C)$ has at least three vertices. If $O_{i, i+1}^{*}(C)$ has at least three vertices, then such vertices also belong to $O_{i+1, i+1}^{*}(C)$, and the claim follows; otherwise, if $O_{i, i+1}^{*}(C)$ is a single vertex, then it has at least three incident edges connecting it to $C$, two of which are subdivided, and the claim follows as such subdivision vertices belong to $O_{i+1, i+1}^{*}(C)$; otherwise $O_{i, i+1}^{*}(C)$ is a single edge and each of its end-vertices has at least two incident edges, hence at least two such edges are subdivided, and the claim follows as such subdivision vertices belong to $O_{i+1, i+1}^{*}(C)$.

Second, we prove that $O_{i+1, i+1}^{*}(C)$ is 2 -connected. We observe that the cycle composed of the edges connecting two consecutive vertices of $L$ passes exactly once through all the subdivision vertices inserted inside $C$ and through all the vertices of $V_{i+1}$. Namely, each subdivision vertex is inserted inside $L$ exactly once, at the only time the edge it subdivides is encountered when processing $\mathcal{O}$; each vertex of $V_{i+1}$ that is not a cut-vertex and such that $O_{i, i+1}^{*}(C)$ has at least three vertices is inserted inside $L$ when its incident edges connecting it to $C$ are encountered; however, the vertex is inserted into $L$ exactly once, by construction; finally, each cut-vertex of $V_{i+1}$ in $O_{i, i+1}^{*}(C)$ or, if $O_{i, i+1}^{*}(C)$ has at most two vertices, each vertex of $O_{i, i+1}^{*}(C)$ is inserted into $L$ exactly once, at the only time its last incident edge in $\mathcal{O}$ is en- 
countered when processing $\mathcal{O}$. As there is a cycle passing through all the vertices of $O_{i+1, i+1}^{*}(C)$, then $O_{i+1, i+1}^{*}(C)$ is 2 -connected.

After step $k$ of the algorithm, $G^{*}=G_{k+1}^{*}$ is a maximal plane graph that is level-2connected and that contains a subgraph $G^{\prime}$ such that $G^{\prime}$ is a 1-subdivision of $G$ (observe that each edge of $G$ connecting two vertices on two distinct levels is subdivided at most once, while no edge of $G$ connecting two vertices on the same level is ever subdivided). Finally, since the number of subdivision vertices introduced by the algorithm is at most the number of edges of $G$, then $G^{*}$ has $O(n)$ vertices, thus proving the lemma.

We now state some simple lemmata on the topologies of certain subgraphs of a level2-connected graph. Such lemmata will be used in some of the proofs of Section 3.

Let $G$ be a level-2-connected graph and let $C$ be a cycle delimiting the outer face of a 2-connected component of the $i$-th level of $G$. Let $G_{C}$ be the subgraph of $G$ inside or on the border of $C$. We have the following lemma.

Lemma $2 G_{C}$ is a level-2-connected graph.

Proof: By construction $C$ delimits the outer face of a 2-connected component of the $i$-th level of $G$. Hence, if the $j$-th level of $G_{C}$ does not induce a set of vertex-disjoint 2-connected components, then the $(i+j-1)$-th level of $G_{C}$ does not induce a set of vertex-disjoint 2-connected components, too. It follows that $G_{C}$ is a level-2-connected graph.

Let $G$ be a level-2-connected graph and let $(u, v)$ be a chord of $G$. Let $V_{1}$ and $V_{2}$ be the vertex sets of the two connected components of $G$ which are obtained by removing $u$, $v$, and their incident edges. We have the following lemma.

Lemma $3 G\left[V_{1} \cup\{u, v\}\right]$ and $G\left[V_{2} \cup\{u, v\}\right]$ are level-2-connected graphs.

Proof: First, $G\left[V_{1} \cup\{u, v\}\right]$ and $G\left[V_{2} \cup\{u, v\}\right]$ are 2-connected and internally-triangulated as $G$ is. Let $k_{1}$ and $k_{2}$ be the number of levels of $G\left[V_{1} \cup\{u, v\}\right]$ and $G\left[V_{2} \cup\{u, v\}\right]$, respectively. Denote by $C_{1}$ and $C_{2}$ the cycles delimiting the outer faces of $G\left[V_{1} \cup\{u, v\}\right]$ and $G\left[V_{2} \cup\{u, v\}\right]$, respectively. The subgraph of $G\left[V_{1} \cup\{u, v\}\right]$ (resp. of $G\left[V_{2} \cup\{u, v\}\right]$ ) induced by the vertices on the first level of $G\left[V_{1} \cup\{u, v\}\right]$ (resp. of $G\left[V_{2} \cup\{u, v\}\right]$ ) has exactly one 2-connected component. Further, for each $2 \leq i \leq k_{1}$ (resp. for each $1 \leq i \leq k_{2}$ ), the subgraph of $G\left[V_{1} \cup\{u, v\}\right]$ (resp. of $G\left[V_{2} \cup\{u, v\}\right]$ ) induced by the vertices on the $i$-th level of $G\left[V_{1} \cup\{u, v\}\right]$ (resp. of $G\left[V_{2} \cup\{u, v\}\right]$ ) is composed of a set of 2-connected components that are exactly the 2-connected components of $G\left[V_{i}\right]$ inside $C_{1}$ (resp. inside $C_{2}$ ). It follows that such 2-connected components are pairwise vertex-disjoint and have each at least three vertices. Hence, $G\left[V_{1} \cup\{u, v\}\right]$ and $G\left[V_{2} \cup\{u, v\}\right]$ are level-2-connected graphs.

Let $G$ be a level-2-connected graph and let $G_{i}^{+}$and $G_{i}^{*}$ be two distinct connected components induced by the $i$-th level of $G$. We have the following property.

Property 1 No edge of $G$ connects a vertex of $G_{i}^{+}$and a vertex of $G_{i}^{*}$. 


\section{Floored Graphs}

In this section we define floored graphs and show how to decompose a floored graph into two smaller floored graphs. A floored graph (see Fig. 3) is a graph $G$ with one distinguished vertex or edge on the outer face and whose vertex set is partitioned into sets $F_{1}, F_{2}, \ldots, F_{k}$ that induce subgraphs of $G$, called floors, satisfying the topological properties described below. More formally, a floored graph is a triple $(G, f, g)$, where $G(V, E)$ is a 2-connected internally-triangulated plane graph, $f$ is a function $f: V \rightarrow \mathbb{N}$, where $F_{i}=f^{-1}(i)$, for $i=1, \ldots, k$ (denote by $k$ the largest integer such that $f^{-1}(k) \neq \emptyset$ ), and $g$ is an edge in $E$ or a vertex in $V$, such that the following conditions are satisfied:

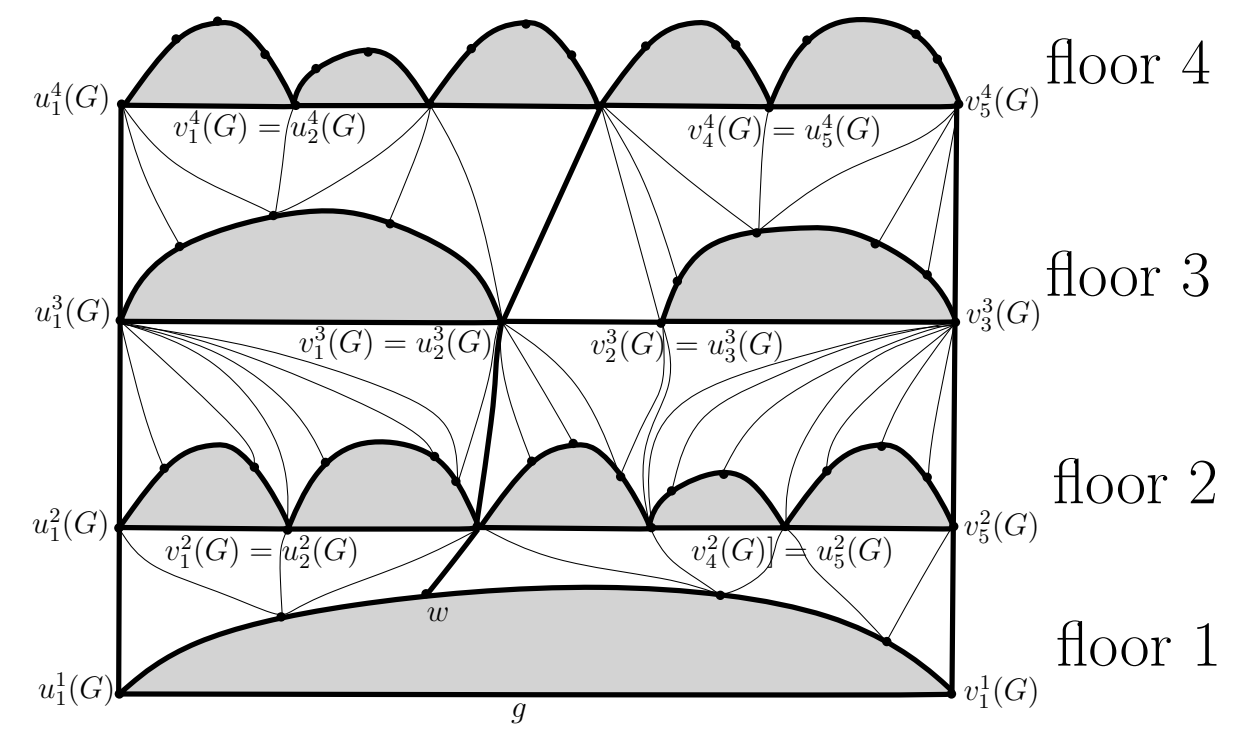

Figure 3: A floored graph $(G, f, g)$ with 4 floors. Level-2-connected graphs are gray. Their outer faces are shown by thick lines. The borders of $(G, f, g)$ are shown by thick lines. In this example $G\left[F_{1}\right]$ is a level-2-connected graph. Hence, $g=\left(u_{1}^{1}(G), v_{1}^{1}(G)\right)$. A raising path starting at $w$ is shown by thick lines.

C1: Graph $G\left[F_{1}\right]$ is either a vertex on the outer face of $G$ (then $g$ is such a vertex and let $u_{1}^{1}(G)=v_{1}^{1}(G)=g$ ), or an edge on the outer face of $G$ (then $g$ is such an edge and let $g=\left(u_{1}^{1}(G), v_{1}^{1}(G)\right)$, where $G$ is to the left of $g$ when traversing it from $u_{1}^{1}(G)$ to $v_{1}^{1}(G)$ ), or a level-2-connected graph (then $g=\left(u_{1}^{1}(G), v_{1}^{1}(G)\right)$ is an edge of $G\left[F_{1}\right]$ on the outer face of $G$, where $G$ is to the left of $g$ when traversing it from $u_{1}^{1}(G)$ to $\left.v_{1}^{1}(G)\right) . G\left[F_{1}\right]$ is the first floor of $(G, f, g)$;

C2: For each $2 \leq i \leq k-1$, graph $G\left[F_{i}\right]$ is composed of a sequence $G_{1}^{i}, G_{2}^{i}, \ldots, G_{x(i)}^{i}$ of graphs which are either single edges or level-2-connected graphs, where $x(i) \geq 1$, such that: (i) $G_{1}^{i}$ has a vertex $u_{1}^{i}(G)$ on the outer face of $G$; (ii) $G_{x(i)}^{i}$ has a vertex $v_{x(i)}^{i}(G)$ on the outer face of $G$; (iii) $G_{j}^{i}$ has a vertex $v_{j}^{i}(G)$ coincident with a vertex $u_{j+1}^{i}(G)$ of $G_{j+1}^{i}$, for $1 \leq j \leq x(i)-1$; such a vertex is on the outer faces of both graphs; (iv) $G_{j}^{i}$ and $G_{j+1}^{i}$ lie each one in the outer face of the other one, for $1 \leq j \leq x(i)-1 ;(\mathrm{v}) G_{j}^{i}$ and $G_{l}^{i}$ do not share any vertex, for $l \neq j-1, j+1$; (vi) edge $\left(u_{j}^{i}(G), v_{j}^{i}(G)\right)$ exists and is on the outer face of $G_{j}^{i}$, for $1 \leq j \leq x(i)$; (vii) $G_{j}^{i}$ 
(if such a graph is not an edge) is to the left of $\left(u_{j}^{i}(G), v_{j}^{i}(G)\right)$ when traversing it from $u_{j}^{i}(G)$ to $v_{j}^{i}(G) . G\left[F_{i}\right]$ is the $i$-th floor of $(G, f, g)$;

C3: Graph $G\left[F_{k}\right]$ is either a single vertex $u_{1}^{k}(G)=v_{x(k)}^{k}(G)$ on the outer face of $G$, or a sequence $G_{1}^{k}, G_{2}^{k}, \ldots, G_{x(k)}^{k}$ of graphs which are either single edges or level-2connected graphs, where $x(k) \geq 1$, such that properties (i)-(vii) of Condition C2 hold (in such a case $u_{1}^{k}(G)$ and $v_{x(k)}^{k}(G)$ are defined as in such properties). $G\left[V_{k}\right]$ is the last floor of $(G, f, g)$;

C4: $G$ contains no edge connecting the $i_{1}$-th floor and the $i_{2}$-th floor of $(G, f, g)$, with $i_{2} \neq i_{1}-1, i_{1}+1$

C5: Any floor is in the outer face of each other floor; and

C6: Paths $B_{1}=\left(u_{1}^{1}(G), u_{1}^{2}(G), \ldots, u_{1}^{F}(G)\right)$ and $B_{2}=\left(v_{1}^{1}(G), v_{x(2)}^{2}(G), \ldots, v_{x(k)}^{k}(G)\right)$ exist and are on the outer face of $G$. Such paths are called the borders of $(G, f, g)$. If $(G, f, g)$ has one floor, then $B_{1}$ and $B_{2}$ are single vertices.

Level-2-connected graphs can be easily turned into floored graphs, as shown in the following.

Lemma 4 Let $G(V, E)$ be a level-2-connected graph. Let $f$ be the function $f: V \rightarrow \mathbb{N}$ such that $f(z)=1$, for every $z \in V$. Let $g=\left(u_{1}^{1}, v_{1}^{1}\right)$ be any edge on the outer face of $G$, where $G$ is to the left of $g$ when traversing such an edge from $u_{1}^{1}$ to $v_{1}^{1}$. Then, $(G, f, g)$ is a floored graph.

Proof: Condition $\mathrm{C} 1$ is satisfied since $G\left[F_{1}\right]=G$ is a level-2-connected graph and since $g=\left(u_{1}^{1}, v_{1}^{1}\right)$ is an edge on the outer face of $G$, where $G$ is to the left of $g$ when traversing such an edge from $u_{1}^{1}$ to $v_{1}^{1}$. Conditions $\mathrm{C} 2, \mathrm{C} 4$, and $\mathrm{C} 5$ are satisfied since $(G, f, g)$ has exactly one floor. Condition C3 is satisfied since $G\left[F_{1}\right]=G$ is a single level2-connected graph (thus satisfying C3(iii)-(v)), since $u_{1}^{1}$ and $v_{1}^{1}$ are on the outer face of $G\left[F_{1}\right]$ (thus satisfying C3(i) and C3(ii)), since edge $\left(u_{1}^{1}, v_{1}^{1}\right)$ exists (thus satisfying C3(vi)), and since $G$ is to the left of $g$ when traversing such an edge from $u_{1}^{1}$ to $v_{1}^{1}$ (thus satisfying C3(vii)). Condition C6 is satisfied since vertices $u_{1}^{1}$ and $v_{1}^{1}$ are the borders of $(G, f, g)$.

We have the following structural lemma (see Fig. 5).

Lemma 5 Let $(G, f, g)$ be a floored graph. Then, exactly one of the following assertions is true. (1) $G\left[F_{1}\right]$ is vertex $g$ and $\left(g, u_{1}^{2}(G), v_{x(2)}^{2}(G)\right)$ is an internal face of $G$. (2) $G\left[F_{1}\right]$ is vertex $g$ and vertices $g, u_{1}^{2}(G)$, and $v_{x(2)}^{2}(G)$ are not on the same internal face of $G$. (3) $G\left[F_{1}\right]$ is an edge $g=\left(u_{1}^{1}(G), v_{1}^{1}(G)\right)$. (4) $G\left[F_{1}\right]$ is a level-2-connected graph and the vertex $w$ of $G$ that forms an internal face with $g$ is on the outer face of $G\left[F_{1}\right]$. (5) $G\left[F_{1}\right]$ is a level-2-connected graph and the vertex $w_{1}$ of $G$ that forms an internal face with $g$ is not on the outer face of $G\left[F_{1}\right]$.

Proof: First, observe that if assertion (x) is satisfied, then assertion (y) is not satisfied, for any choice of $x$ and $y$ such that $1 \leq x, y \leq 5$ and $x \neq y$. Suppose that $G\left[F_{1}\right]$ is vertex $g$. Then, all its neighbors are on the second floor of $(G, f, g)$, since $(G, f, g)$ satisfies Condition C4. Observe that $u_{1}^{2}(G)$ and $v_{x(2)}^{2}(G)$ are neighbors of $g$, since $(G, f, g)$ satisfies Condition 
C6. If $g$ has no neighbor different from $u_{1}^{2}(G)$ and $v_{x(2)}^{2}(G)$, then $\left(g, u_{1}^{2}(G), v_{x(2)}^{2}(G)\right)$ is an internal face of $G$, since $G$ is internally-triangulated, and assertion (1) is satisfied. If $g$ has neighbors different from $u_{1}^{2}(G)$ and $v_{x(2)}^{2}(G)$, then by planarity $\left(g, u_{1}^{2}(G), v_{x(2)}^{2}(G)\right.$ is not an internal face of $G$, and assertion (2) is satisfied. If $G\left[F_{1}\right]$ is an edge, then assertion (3) is satisfied. If $G\left[F_{1}\right]$ is a level-2-connected graph, then, by planarity and by the fact that $(G, f, g)$ satisfies Condition $\mathrm{C} 5$, the vertex $w$ that forms an internal face of $G$ with $g$ is in $G\left[F_{1}\right]$. If $w$ is on the outer face of $G\left[F_{1}\right]$, then assertion (4) is satisfied, otherwise assertion (5) is satisfied.

We now define and study raising paths, that are paths that will be used in order to split floored graphs into smaller floored graphs. Let $(G, f, g)$ be a floored graph and let $w \neq g$ be a vertex on the outer face of the $i$-th floor of $G$, for any $1 \leq i \leq k$. A raising path starting at $w$ is a path $R(w)=\left(w_{1}=w, w_{2}, \ldots, w_{y}\right)$ such that $f\left(w_{x}\right)=f\left(w_{x-1}\right)+1$, for every $1 \leq x \leq y-1$, and such that, if a vertex $w_{x}$ belongs to the border $B_{1}$ (resp. to $B_{2}$ ), then all the vertices after $w_{x}$ in $R(w)$ belong to $B_{1}$ (resp. to $B_{2}$ ). We have the following.

Lemma 6 Let $(G, f, g)$ be a floored graph. For every vertex $w$ of the outer face of a floor of $G$ different from the last floor, there exists a vertex $z$ on the outer face of $G\left[F_{f(w)+1}\right]$ and adjacent to $w$.

Proof: If $w$ belongs to $B_{1}$ (resp. $B_{2}$ ), the next vertex on $B_{1}$ (resp. on $B_{2}$ ) is chosen as

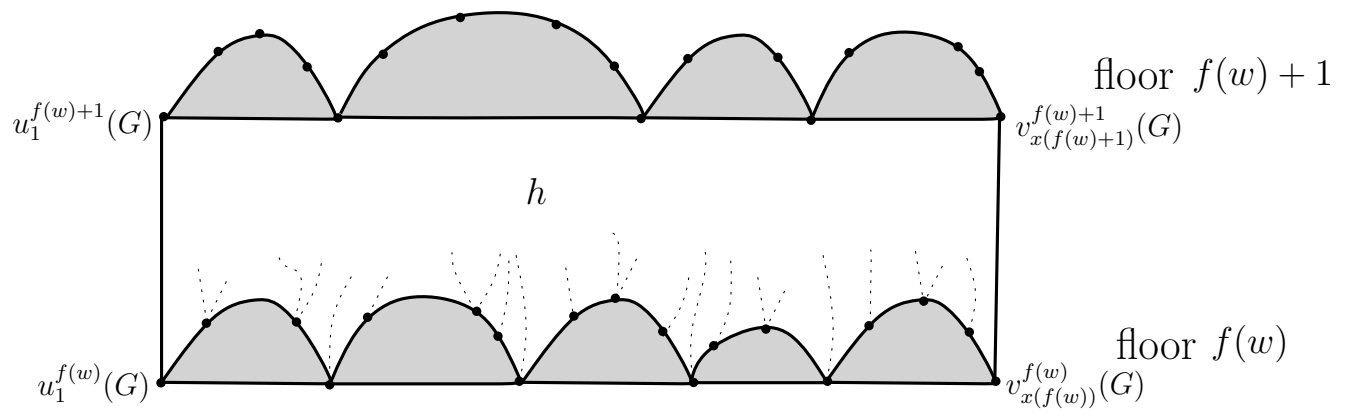

Figure 4: Graph $H$, shown by thick lines and gray regions, representing the level-2connected graphs in the $f(w)$-th floor and in the $(f(w)+1)$-th floor.

$z$. Otherwise, $G\left[F_{f(w)}\right]$ is not a single edge, hence it is a graph composed of a sequence of edges and level-2-connected graphs, and $w$ is internal to $G$. Then, consider the subgraph $H$ of $G$ composed of $G\left[F_{f(w)}\right]$, of $G\left[F_{f(w)+1}\right]$, and of edges $\left(u_{1}^{f(w)}(G), u_{1}^{f(w)+1}(G)\right)$ and $\left(v_{x(f(w))}^{f(w)}(G), v_{x(f(w)+1)}^{f(w)+1}(G)\right)$ (see Fig. 4). Denote by $h$ the only internal face of $H$ with more than three incident vertices. Every vertex on the outer face of $G\left[F_{f(w)}\right]$ is incident to $h$ in $H$, by Condition $\mathrm{C} 1$ if $f(w)=1$ and by Condition C2 if $f(w)>1$; since $G$ is internally-triangulated, every such a vertex (and hence $w$ ) is adjacent to at least one vertex in $G\left[F_{f(w)+1}\right]$ (let $z$ be a vertex of $G\left[F_{f(w)+1}\right]$ adjacent to $w$ ). By planarity, $z$ is on the outer face of $G\left[F_{f(w)+1}\right]$. The lemma follows.

Corollary 1 Let $(G, f, g)$ be a floored graph. For every vertex $w \neq g$ on the outer face of a floor of $G$, there exists a raising path starting at $w$. 
Proof: We prove the statement by reverse induction on the floor of $w$. If $f(w)=k$, then $R(w)$ consists only of vertex $w$. If $f(w)<k$, then consider any vertex $z$ on the outer face of $G\left[F_{f(w)+1}\right]$ and adjacent to $w$. Such a vertex $z$ exists by Lemma 6 . Then, induction applies and there exists a raising path $R(z)$ starting at $z$. Concatenating edge $(w, z)$ with $R(z)$ results in the desired path $R(w)$.

Suppose that a raising path $R(w)$ shares vertices with $B_{1}$. Then, path $R(w) \backslash B_{1}$ is the subpath of $R(w)$ starting at $w$ and ending at the first vertex $z$ shared by $R(w)$ and $B_{1}\left(z\right.$ is in $\left.R(w) \backslash B_{1}\right)$. Further, $B_{1} \backslash R(w)$ is the subpath of $B_{1}$ starting at $u_{1}^{1}(G)$ and ending at the first vertex $z$ shared by $R(w)$ and $B_{1}$ ( $z$ is in $B_{1} \backslash R(w)$ ). If $R(w)$ shares vertices with $B_{2}$, then $R(w) \backslash B_{2}$ and $B_{2} \backslash R(w)$ are defined analogously.

Let $(G, f, g)$ be a floored graph such that $G$ has more than three vertices. Denote by $P$ the subpath of the outer face of $G$ between $u_{1}^{k}(G)$ and $v_{x(k)}^{k}(G)$ and not containing $g$. Given a vertex $w_{y}$ in $P$, let $P_{1}\left(w_{y}\right)$ (resp. $P_{2}\left(w_{y}\right)$ ) be the subpath of $P$ between $u_{1}^{k}(G)$ and $w_{y}$ (resp. between $w_{y}$ and $\left.v_{x(k)}^{k}(G)\right)$.

We now discuss how to use a raising path to split a floored graph $(G, f, g)$ into two floored graphs. We distinguish five cases, according to the five mutually-exclusive assertions of Lemma 5.

Case 1. $G\left[F_{1}\right]$ is vertex $g$ and $\left(g, u_{1}^{2}(G), v_{x(2)}^{2}(G)\right)$ is an internal face of $G$. See Fig. 5.a. Actually this case does not use a raising path, but changes $G$ by removing one of its vertices still obtaining a floored graph in which $g$ is now an edge. Let $\left(G^{\prime}, f^{\prime}, g^{\prime}\right)$ be the triple defined as follows. $G^{\prime}\left(V^{\prime}, E^{\prime}\right)$ is the graph obtained from $G$ by removing vertex $g$ and its incident edges $\left(g, u_{1}^{2}(G)\right)$ and $\left(g, v_{x(2)}^{2}(G)\right), f^{\prime}(w)=f(w)-1$, for each vertex $w \in V^{\prime}$, and $g^{\prime}=\left(u_{1}^{2}(G), v_{x(2)}^{2}(G)\right)$.

Lemma $7\left(G^{\prime}, f^{\prime}, g^{\prime}\right)$ is a floored graph.

Proof: We prove that Condition C1 is satisfied by $\left(G^{\prime}, f^{\prime}, g^{\prime}\right)$. Edge $\left(u_{1}^{1}\left(G^{\prime}\right), v_{1}^{1}\left(G^{\prime}\right)\right)=$ $g^{\prime}$ exists and is on the outer face of $G^{\prime}$. Further, such an edge has $G^{\prime}$ to its left when traversed from $u_{1}^{1}\left(G^{\prime}\right)$ to $v_{1}^{1}\left(G^{\prime}\right)$. Hence, since $(G, f, g)$ satisfies Condition $\mathrm{C} 2, G^{\prime}\left[F_{1}^{\prime}\right]$ is either a single edge or a level-2-connected graph such that $g^{\prime}$ is on the outer face of $G^{\prime}$ and $g^{\prime}$ has $G^{\prime}$ to its left when traversed from $u_{1}^{1}\left(G^{\prime}\right)$ to $v_{1}^{1}\left(G^{\prime}\right)$.

Conditions C2, C3, C4, and C5 are satisfied by $\left(G^{\prime}, f^{\prime}, g^{\prime}\right)$ since $(G, f, g)$ satisfies Conditions C2, C3, C4, and C5, respectively (observe that the $i$-th floor of $\left(G^{\prime}, f^{\prime}, g^{\prime}\right)$ is the $(i+1)$-th floor of $(G, f, g)$ and that the last floor of $\left(G^{\prime}, f^{\prime}, g^{\prime}\right)$ is the last floor of $(G, f, g))$.

Conditions C6 is satisfied by $\left(G^{\prime}, f^{\prime}, g^{\prime}\right)$ since $(G, f, g)$ satisfies Condition C6 and the borders $B_{1}\left(G^{\prime}\right)$ and $B_{2}\left(G^{\prime}\right)$ of $\left(G^{\prime}, f^{\prime}, g^{\prime}\right)$ are obtained from the corresponding borders $B_{1}(G)$ and $B_{2}(G)$ of $(G, f, g)$ by removing vertices $u_{1}^{1}(G)$ and $v_{1}^{1}(G)$, respectively.

Case 2. $G\left[F_{1}\right]$ is vertex $g$ and vertices $g, u_{1}^{2}(G)$, and $v_{x(2)}^{2}(G)$ are not on the same internal face of $G$. See Fig. 5.b. Consider any edge $(g, w)$ internal to $G$. Observe that such an edge exists, as $G$ is internally-triangulated. Consider any raising path $R(w)$ starting at $w$.

If $R(w)$ does not share vertices with $B_{1}$ and $B_{2}$, then let $w_{y}$ be the last vertex of $R(w)$. Let $G^{\prime}\left(V^{\prime}, E^{\prime}\right)$ be the subgraph of $G$ inside or on the border of cycle $B_{1} \cup P_{1}\left(w_{y}\right) \cup$ $R(w) \cup(g, w)$ and let $G^{\prime \prime}\left(V^{\prime \prime}, E^{\prime \prime}\right)$ be the subgraph of $G$ inside or on the border of cycle $B_{2} \cup P_{2}\left(w_{y}\right) \cup R(w) \cup(g, w)$. Let $f^{\prime}(z)=f(z)$, for each vertex $z \in V^{\prime}$, and let $f^{\prime \prime}(z)=f(z)$, 


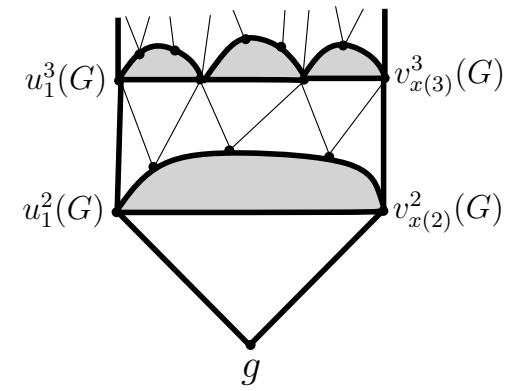

(a)

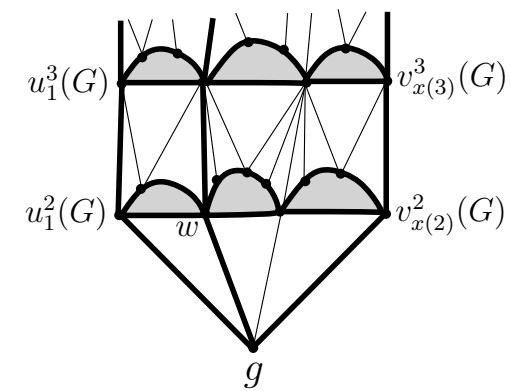

(b)

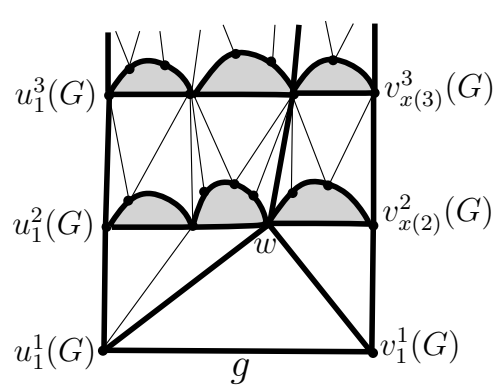

(c)

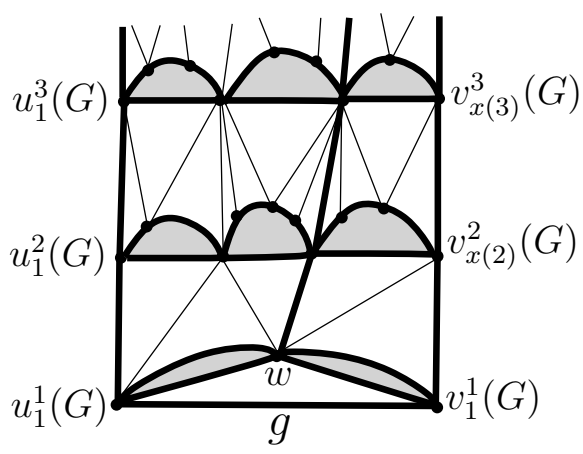

(d)

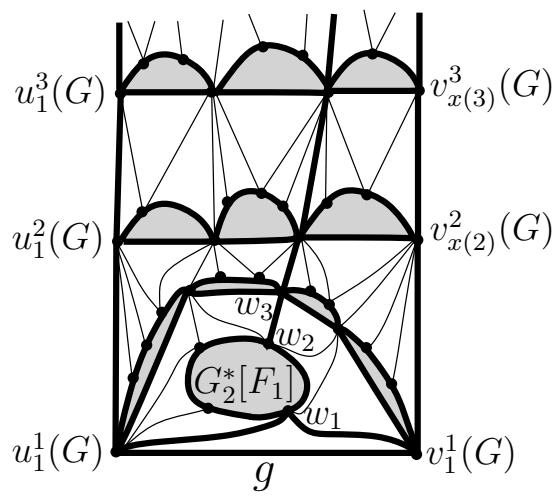

(e)

Figure 5: Illustration for Cases 1-5. The borders of $(G, f, g)$, of $\left(G^{\prime}, f^{\prime}, g^{\prime}\right)$, and of $\left(G^{\prime \prime}, f^{\prime \prime}, g^{\prime \prime}\right)$ and the cycles delimiting the outer faces of the floors of $(G, f, g)$, of $\left(G^{\prime}, f^{\prime}, g^{\prime}\right)$, and of $\left(G^{\prime \prime}, f^{\prime \prime}, g^{\prime \prime}\right)$ are shown by thick lines. (a) Case 1. (b) Case 2. (c) Case 3. (d) Case 4. (e) Case 5.

for each vertex $z \in V^{\prime \prime}$. Finally, let $g^{\prime}=g$ and $g^{\prime \prime}=g$. If $R(w)$ shares vertices with $B_{1}$ (the case in which it shares vertices with $B_{2}$ being analogous), let $G^{\prime}\left(V^{\prime}, E^{\prime}\right)$ be the subgraph of $G$ inside or on the border of cycle $\left(B_{1} \backslash R(w)\right) \cup\left(R(w) \backslash B_{1}\right) \cup(g, w)$ and let $G^{\prime \prime}\left(V^{\prime \prime}, E^{\prime \prime}\right)$ be the subgraph of $G$ inside or on the border of cycle $P \cup R(w) \cup(g, w) \cup B_{2}$. Let $f^{\prime}(z)=f(z)$, for each vertex $z \in V^{\prime}$, and let $f^{\prime \prime}(z)=f(z)$, for each vertex $z \in V^{\prime \prime}$. Finally, let $g^{\prime}=g$ and $g^{\prime \prime}=g$.

Lemma $8\left(G^{\prime}, f^{\prime}, g^{\prime}\right)$ and $\left(G^{\prime \prime}, f^{\prime \prime}, g^{\prime \prime}\right)$ are floored graphs.

Proof: We prove that $\left(G^{\prime}, f^{\prime}, g^{\prime}\right)$ is a floored graph, the proof that $\left(G^{\prime \prime}, f^{\prime \prime}, g^{\prime \prime}\right)$ is a floored graph being analogous.

Condition C1 is satisfied by $\left(G^{\prime}, f^{\prime}, g^{\prime}\right)$ as $g^{\prime}$ is a vertex on the outer face of $G^{\prime}$, since $g$ is on the outer face of $G$ by Condition $\mathrm{C} 1$ on $(G, f, g)$.

To prove that Condition $\mathrm{C} 2$ is satisfied by $\left(G^{\prime}, f^{\prime}, g^{\prime}\right)$, we have to prove that the $i$-th floor of $\left(G^{\prime}, f^{\prime}, g^{\prime}\right)$ satisfies Conditions C2(i)-(vii), for each $2 \leq i \leq k^{\prime}-1$, where $k^{\prime}$ is the number of floors of $\left(G^{\prime}, f^{\prime}, g^{\prime}\right)$. We distinguish some cases. If $R(w)$ shares a vertex $w_{x}$ with $B_{1}$, where $f\left(w_{x}\right)<i$ (see Fig. 6.a, with $i \geq 5$ and $\left.w_{x}=u_{1}^{4}(G)\right)$, then $\left(G^{\prime}, f^{\prime}, g^{\prime}\right)$ has strictly less then $i$ floors and there is nothing to prove. If $R(w)$ shares a vertex $w_{x}$ with $B_{2}$, where $f\left(w_{x}\right) \leq i$ (see Fig. 6.b, where $i \geq 4$ and $w_{x}=v_{x(4)}^{4}(G)$ ), then the $i$-th floor of $\left(G^{\prime}, f^{\prime}, g^{\prime}\right)$ is the $i$-th floor of $(G, f, g)$, hence it satisfies Conditions C2(i)-(vii) as the $i$-th floor of $(G, f, g)$ does. Otherwise, $R(w)$ partitions the $i$-th floor of $(G, f, g)$ into two subgraphs, 


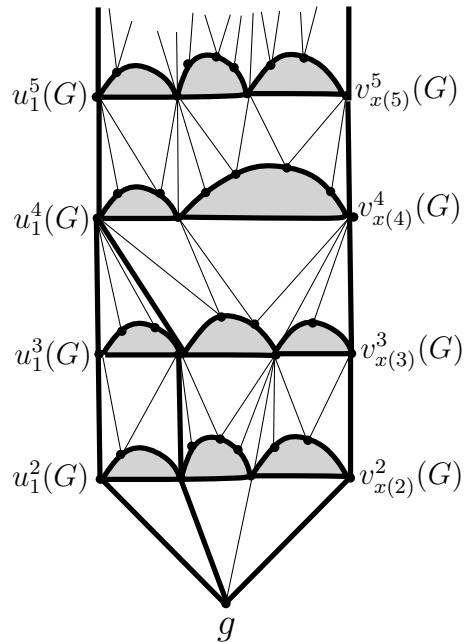

(a)

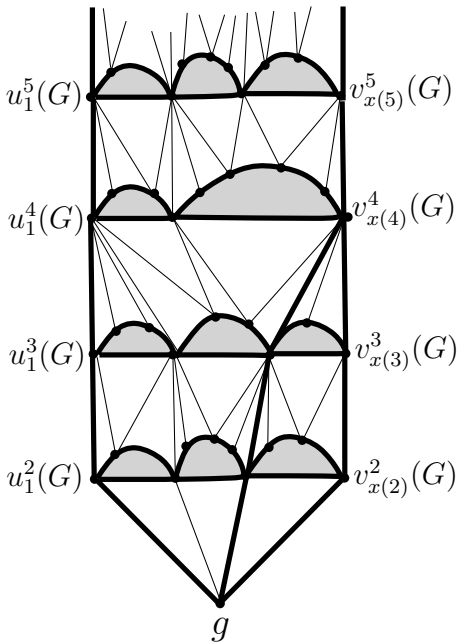

(b)

Figure 6: Illustration for Cases 1-5. The borders of $(G, f, g)$, of $\left(G^{\prime}, f^{\prime}, g^{\prime}\right)$, and of $\left(G^{\prime \prime}, f^{\prime \prime}, g^{\prime \prime}\right)$ and the cycles delimiting the outer faces of the floors of $(G, f, g)$, of $\left(G^{\prime}, f^{\prime}, g^{\prime}\right)$, and of $\left(G^{\prime \prime}, f^{\prime \prime}, g^{\prime \prime}\right)$ are shown by thick lines. (a) Case 1. (b) Case 2. (c) Case 3. (d) Case 4. (e) Case 5.

one belonging to $G^{\prime}$ and the other one belonging to $G^{\prime \prime}$ (see Fig. 6.a and Fig. 6.b, with $2 \leq i \leq 3)$. Observe that vertex $w_{i-1}$ of $R(w)=\left(w=w_{1}, w_{2}, \ldots, w_{k-1}\right)$, which lies on the $i$-th floor of $(G, f, g)$ and hence on the $i$-th floor of $\left(G^{\prime}, f^{\prime}, g^{\prime}\right)$, is incident to a vertex of the $(i-1)$-th floor of $(G, f, g)$ (namely, either $w_{i-2}$ if $i>2$ or $g$ if $i=2$ ) and to a vertex of the $(i+1)$-th floor of $(G, f, g)$ (since the $i$-th floor is not the last floor of $\left(G^{\prime}, f^{\prime}, g^{\prime}\right)$, as $\left.i<k^{\prime}\right)$. Hence, by planarity $w_{i}$ is a vertex $u_{l}^{i}(G)$, for some $2 \leq l \leq x(i)-1$. It follows that the $i$-th floor of $\left(G^{\prime}, f^{\prime}, g^{\prime}\right)$ is composed of the sequence $G_{1}^{i}, G_{2}^{i}, \ldots G_{l-1}^{i}$ of graphs which are the first $l-1$ graphs in the sequence $G_{1}^{i}, G_{2}^{i}, \ldots G_{x(i)}^{i}$ of graphs composing the $i$-th floor of $(G, f, g)$. Hence, the $i$-th floor of $\left(G^{\prime}, f^{\prime}, g^{\prime}\right)$ satisfies Conditions C2(i)-(vii) as the $i$-th floor of $(G, f, g)$ does.

The proof that Condition C3 is satisfied by $\left(G^{\prime}, f^{\prime}, g^{\prime}\right)$ is analogous to the one that Condition $\mathrm{C} 2$ is satisfied by $(G, f, g)$.

To prove that Conditions $\mathrm{C} 4$ is satisfied by $\left(G^{\prime}, f^{\prime}, g^{\prime}\right)$, observe that if an edge exists connecting the $i_{1}$-th floor and the $i_{2}$-th floor of $\left(G^{\prime}, f^{\prime}, g^{\prime}\right)$, with $i_{2} \neq i_{1}-1, i_{1}+1$, then such an edge also connects the $i_{1}$-th floor and the $i_{2}$-th floor of $(G, f, g)$, as $f^{\prime}(z)=f(z)$, for each vertex $z \in V^{\prime}$; however, this is not possible since Condition C4 is satisfied by $(G, f, g)$.

Condition C5 is satisfied by $\left(G^{\prime}, f^{\prime}, g^{\prime}\right)$ since $G^{\prime}\left[F_{i}^{\prime}\right]$ is a subgraph of $G\left[F_{i}\right]$, for each $1 \leq i \leq k^{\prime}$, since the embedding of $G^{\prime}$ is the same embedding that such a graph has in $G$, and since Condition C5 is satisfied by $(G, f, g)$.

If $R(w)$ and $B_{1}$ do not share vertices, then Condition C6 is satisfied by $\left(G^{\prime}, f^{\prime}, g^{\prime}\right)$ as $B_{1}$ and $\left(u_{1}^{1}(G), w\right) \cup R(w)$ are the borders of $\left(G^{\prime}, f^{\prime}, g^{\prime}\right)$; otherwise, Condition C6 is satisfied by $\left(G^{\prime}, f^{\prime}, g^{\prime}\right)$ as $B_{1} \backslash R(w)$ and $\left(u_{1}^{1}(G), w\right) \cup\left(R(w) \backslash B_{1}\right)$ are the borders of $\left(G^{\prime}, f^{\prime}, g^{\prime}\right)$.

Case 3. $G\left[F_{1}\right]$ is an edge $g=\left(u_{1}^{1}(G), v_{1}^{1}(G)\right)$. See Fig. 5.c. Consider the vertex $w$ of $G$ that forms an internal face with $g$. Notice that such a vertex exists as $G$ is internallytriangulated and belongs to the second floor of $G$ (by the fact that $G\left[F_{1}\right]$ is an edge and 
by Condition C4).

If $w$ belongs to $B_{2}$, that is $w=v_{x(2)}^{2}(G)$, then let $G^{\prime}\left(V^{\prime}, E^{\prime}\right)$ be the subgraph of $G$ inside or on the border of cycle $B_{1} \cup P \cup\left(B_{2} \backslash\left\{\left(v_{1}^{1}(G), w\right)\right\}\right) \cup\left(u_{1}^{1}(G), w\right)$, let $f^{\prime}(z)=f(z)$, for each vertex $z \in V^{\prime}$, and let $g^{\prime}=u_{1}^{1}(G)$. If $w$ belongs to $B_{1}$, that is $w=u_{1}^{2}(G)$, then let $G^{\prime \prime}\left(V^{\prime \prime}, E^{\prime \prime}\right)$ be the subgraph of $G$ inside or on the border of cycle $B_{2} \cup P \cup\left(B_{1} \backslash\right.$ $\left.\left\{\left(u_{1}^{1}(G), w\right)\right\}\right) \cup\left(v_{1}^{1}(G), w\right)$, let $f^{\prime \prime}(z)=f(z)$, for each vertex $z \in V^{\prime \prime}$, and let $g^{\prime \prime}=v_{1}^{1}(G)$. If $w$ belongs neither to $B_{1}$ nor to $B_{2}$, then $w$ is an internal vertex of $G$. Consider any raising path $R(w)$ starting at $w$. If $R(w)$ does not share vertices with $B_{1}$ and $B_{2}$, then let $w_{y}$ be the last vertex of $R(w)$. Let $G^{\prime}\left(V^{\prime}, E^{\prime}\right)$ be the subgraph of $G$ inside or on the border of cycle $B_{1} \cup P_{1}\left(w_{y}\right) \cup R(w) \cup\left(u_{1}^{1}(G), w\right)$ and let $G^{\prime \prime}\left(V^{\prime \prime}, E^{\prime \prime}\right)$ be the subgraph of $G$ inside or on the border of cycle $B_{2} \cup P_{2}\left(w_{y}\right) \cup R(w) \cup\left(v_{1}^{1}(G), w\right)$. Let $f^{\prime}(z)=f(z)$, for each vertex $z \in V^{\prime}$, and let $f^{\prime \prime}(z)=f(z)$, for each vertex $z \in V^{\prime \prime}$. Finally, let $g^{\prime}=u_{1}^{1}(G)$ and $g^{\prime \prime}=v_{1}^{1}(G)$. If $R(w)$ shares vertices with $B_{1}$ (the case in which it shares vertices with $B_{2}$ being analogous), let $G^{\prime}\left(V^{\prime}, E^{\prime}\right)$ be the subgraph of $G$ inside or on the border of cycle $\left(B_{1} \backslash R(w)\right) \cup\left(R(w) \backslash B_{1}\right) \cup\left(u_{1}^{1}(G), w\right)$ and let $G^{\prime \prime}\left(V^{\prime \prime}, E^{\prime \prime}\right)$ be the subgraph of $G$ inside or on the border of cycle $P \cup R(w) \cup\left(v_{1}^{1}(G), w\right) \cup B_{2}$. Let $f^{\prime}(z)=f(z)$, for each vertex $z \in V^{\prime}$, and let $f^{\prime \prime}(z)=f(z)$, for each vertex $z \in V^{\prime \prime}$. Finally, let $g^{\prime}=u_{1}^{1}(G)$ and $g^{\prime \prime}=v_{1}^{1}(G)$.

Lemma $9\left(G^{\prime}, f^{\prime}, g^{\prime}\right)$ and $\left(G^{\prime \prime}, f^{\prime \prime}, g^{\prime \prime}\right)$ are floored graphs.

Proof: First consider the case in which $w$ belongs neither to $B_{1}$ nor to $B_{2}$. We prove that $\left(G^{\prime}, f^{\prime}, g^{\prime}\right)$ is a floored graph, the proof that $\left(G^{\prime \prime}, f^{\prime \prime}, g^{\prime \prime}\right)$ is a floored graph being analogous.

Condition C1 is satisfied by $\left(G^{\prime}, f^{\prime}, g^{\prime}\right)$ as $g^{\prime}$ is a vertex on the outer face of $G^{\prime}$, since $u_{1}^{1}(G)$ and $v_{1}^{1}(G)$ are both on the outer face of $G$ by Condition C1 on $(G, f, g)$.

Conditions $\mathrm{C} 2-\mathrm{C} 6$ can be shown to be satisfied by $\left(G^{\prime}, f^{\prime}, g^{\prime}\right)$ exactly as in the proof that Conditions $\mathrm{C} 2-\mathrm{C} 6$ are satisfied by $\left(G^{\prime}, f^{\prime}, g^{\prime}\right)$ when Case 2 has been applied (see Lemma 8).

If $w$ belongs to $B_{2}$, then $\left(G^{\prime \prime}, f^{\prime \prime}, g^{\prime \prime}\right)$ does not exist and $\left(G^{\prime}, f^{\prime}, g^{\prime}\right)$ can be shown to be a floored graph as follows.

Condition C1 is satisfied by $\left(G^{\prime}, f^{\prime}, g^{\prime}\right)$ as $g^{\prime}$ is a vertex on the outer face of $G^{\prime}$, since $u_{1}^{1}(G)$ and $v_{1}^{1}(G)$ are both on the outer face of $G$ by Condition $\mathrm{C} 1$ on $(G, f, g)$.

To prove that Conditions $\mathrm{C} 2$ and $\mathrm{C} 3$ are satisfied by $\left(G^{\prime}, f^{\prime}, g^{\prime}\right)$, we have to prove that the $i$-th floor of $\left(G^{\prime}, f^{\prime}, g^{\prime}\right)$ satisfies Conditions C2(i)-(vii), for each $2 \leq i \leq k^{\prime}$, where $k^{\prime}$ is the number of floors of $\left(G^{\prime}, f^{\prime}, g^{\prime}\right)$. However, the $i$-th floor of $\left(G^{\prime}, f^{\prime}, g^{\prime}\right)$ coincides with the $i$-th floor of $(G, f, g)$, hence it satisfies Conditions C2(i)-(vii) as the $i$-th floor of $(G, f, g)$ does.

Conditions C4 and C5 can be shown to be satisfied by $\left(G^{\prime}, f^{\prime}, g^{\prime}\right)$ exactly as in the proof that Conditions $\mathrm{C} 4$ and $\mathrm{C} 5$ are satisfied by $\left(G^{\prime}, f^{\prime}, g^{\prime}\right)$ when Case 2 has been applied (see Lemma 8).

Condition C6 is satisfied by $\left(G^{\prime}, f^{\prime}, g^{\prime}\right)$ as $B_{1}$ and $\left(u_{1}^{1}(G), w\right) \cup\left(B_{2} \backslash\left\{\left(v_{1}^{1}(G), w\right\}\right.\right.$ are the borders of $\left(G^{\prime}, f^{\prime}, g^{\prime}\right)$. If $w$ belongs to $B_{1}$, then $\left(G^{\prime}, f^{\prime}, g^{\prime}\right)$ does not exist and $\left(G^{\prime \prime}, f^{\prime \prime}, g^{\prime \prime}\right)$ can be shown to be a floored graph analogously as in the proof that $\left(G^{\prime}, f^{\prime}, g^{\prime}\right)$ is a floored graph when Case 3 has been applied and $w$ belongs to $B_{2}$.

Case 4. $G\left[F_{1}\right]$ is a level-2-connected graph and the vertex $w$ of $G$ that forms an internal face with $g$ is on the outer face of $G\left[F_{1}\right]$. See Fig. 5.d. Consider any raising path $R(w)$ starting at $w$. 
If $R(w)$ does not share vertices with $B_{1}$ and $B_{2}$, then let $w_{y}$ be the last vertex of $R(w)$. Let $G^{\prime}\left(V^{\prime}, E^{\prime}\right)$ be the subgraph of $G$ inside or on the border of cycle $B_{1} \cup P_{1}\left(w_{y}\right) \cup$ $R(w) \cup\left(u_{1}^{1}(G), w\right)$ and let $G^{\prime \prime}\left(V^{\prime \prime}, E^{\prime \prime}\right)$ be the subgraph of $G$ inside or on the border of cycle $B_{2} \cup P_{2}\left(w_{y}\right) \cup R(w) \cup\left(v_{1}^{1}(G), w\right)$. Let $f^{\prime}(z)=f(z)$, for each vertex $z \in V^{\prime}$, and let $f^{\prime \prime}(z)=f(z)$, for each vertex $z \in V^{\prime \prime}$. Finally, let $g^{\prime}=\left(u_{1}^{1}(G), w\right)$ and $g^{\prime \prime}=$ $\left(v_{1}^{1}(G), w\right)$. If $R(w)$ shares vertices with $B_{1}$ (the case in which it shares vertices with $B_{2}$ being analogous), let $G^{\prime}\left(V^{\prime}, E^{\prime}\right)$ be the subgraph of $G$ inside or on the border of cycle $\left(B_{1} \backslash R(w)\right) \cup\left(R(w) \backslash B_{1}\right) \cup\left(u_{1}^{1}(G), w\right)$ and let $G^{\prime \prime}\left(V^{\prime \prime}, E^{\prime \prime}\right)$ be the subgraph of $G$ inside or on the border of cycle $P \cup R(w) \cup\left(v_{1}^{1}(G), w\right) \cup B_{2}$. Let $f^{\prime}(z)=f(z)$, for each vertex $z \in V^{\prime}$, and let $f^{\prime \prime}(z)=f(z)$, for each vertex $z \in V^{\prime \prime}$. Finally, let $g^{\prime}=\left(u_{1}^{1}(G), w\right)$ and $g^{\prime \prime}=\left(v_{1}^{1}(G), w\right)$.

Lemma $10\left(G^{\prime}, f^{\prime}, g^{\prime}\right)$ and $\left(G^{\prime \prime}, f^{\prime \prime}, g^{\prime \prime}\right)$ are floored graphs.

Proof: We prove that $\left(G^{\prime}, f^{\prime}, g^{\prime}\right)$ is a floored graph, the proof that $\left(G^{\prime \prime}, f^{\prime \prime}, g^{\prime \prime}\right)$ is a floored graph being analogous.

Condition $\mathrm{C} 1$ can be shown to be satisfied by $\left(G^{\prime}, f^{\prime}, g^{\prime}\right)$ as follows. If $\left(u_{1}^{1}(G), w\right)$ is an edge on the outer face of $G\left[F_{1}\right]$, then $G^{\prime}\left[F_{1}^{\prime}\right]$ is edge $g^{\prime}=\left(u_{1}^{1}(G), w\right)$, which is on the outer face of $G^{\prime}$. Otherwise, $\left(u_{1}^{1}(G), w\right)$ is a chord of $G\left[F_{1}\right], G^{\prime}\left[F_{1}^{\prime}\right]$ is a level-2-connected graph by Lemma 3, and edge $g^{\prime}=\left(u_{1}^{1}(G), w\right)$ is on the outer face of $G^{\prime}$.

Conditions C2-C5 can be shown to be satisfied by $\left(G^{\prime}, f^{\prime}, g^{\prime}\right)$ exactly as in the proof that Conditions $\mathrm{C} 2-\mathrm{C} 5$ are satisfied by $\left(G^{\prime}, f^{\prime}, g^{\prime}\right)$ when Case 2 has been applied (see Lemma 8).

If $R(w)$ and $B_{1}$ do not share vertices, then Condition C6 is satisfied by $\left(G^{\prime}, f^{\prime}, g^{\prime}\right)$ as $B_{1}$ and $R(w)$ are the borders of $\left(G^{\prime}, f^{\prime}, g^{\prime}\right)$; otherwise, Condition C6 is satisfied by $\left(G^{\prime}, f^{\prime}, g^{\prime}\right)$ as $B_{1} \backslash R(w)$ and $R(w) \backslash B_{1}$ are the borders of $\left(G^{\prime}, f^{\prime}, g^{\prime}\right)$.

Case 5. $G\left[F_{1}\right]$ is a level-2-connected graph and the vertex $w_{1}$ of $G$ that forms an internal face with $g$ is not on the outer face of $G\left[F_{1}\right]$. See Fig. 5.e. By planarity and since $(G, f, g)$ satisfies Condition C5, $w_{1}$ is in $G\left[F_{1}\right]$. Since $w_{1}$ is not on the outer face of $G\left[F_{1}\right]$, it is an internal vertex of $G\left[F_{1}\right]$. By planarity, $w_{1}$ is in the second level of $G\left[F_{1}\right]$. Since $G\left[F_{1}\right]$ is level-2-connected, the subgraph of $G\left[F_{1}\right]$ induced by the vertices in its second level consists of a set of vertex-disjoint 2-connected graphs. Let $G_{2}^{*}\left[F_{1}\right]$ be the one of such graphs $w_{1}$ belongs to. Since $G_{2}^{*}\left[F_{1}\right]$ is 2-connected, its outer face is delimited by a cycle $C$. Orient $C$ so that $G_{2}^{*}\left[F_{1}\right]$ is to its right; let $w_{2}$ be the vertex preceding $w_{1}$ in $C$. Since $G$ is internally-triangulated and since $w_{2}$ is on the outer face of $G_{2}^{*}\left[F_{1}\right], w_{2}$ has at least one incident edge $e$ whose end-vertex $w_{3} \neq w_{2}$ is not in $G_{2}^{*}\left[F_{1}\right]$. We prove that $w_{3}$ is on the outer face of $G\left[F_{1}\right]$. By planarity, $w_{3}$ is not in the $i$-th level of $G\left[F_{1}\right]$, for any $i \geq 3$. If $w_{3}$ is in the second level of $G\left[F_{1}\right]$, then it belongs to a 2-connected component $G_{2}^{+}\left[F_{1}\right]$ of the graph induced by the second level of $G\left[F_{1}\right]$. However, no edge of $G\left[F_{1}\right]$ connects a vertex of $G_{2}^{*}\left[F_{1}\right]$ and a vertex of $G_{2}^{+}\left[F_{1}\right]$, thus $G_{2}^{+}\left[F_{1}\right]=G_{2}^{*}\left[F_{1}\right]$, hence $w_{3}$ belongs to $G_{2}^{*}\left[F_{1}\right]$, contradicting the assumptions on $e$. Then $w_{3}$ is in the first level of $G\left[F_{1}\right]$, that is, it is on the outer face of $G\left[F_{1}\right]$. Consider any raising path $R\left(w_{3}\right)$ starting at $w_{3}$. If $R\left(w_{3}\right)$ does not share vertices with $B_{1}$ and $B_{2}$, then let $w_{y}$ be the last vertex of $R\left(w_{3}\right)$. Let $G^{\prime}\left(V^{\prime}, E^{\prime}\right)$ be the subgraph of $G$ inside or on the border of cycle $B_{1} \cup P_{1}\left(w_{y}\right) \cup R\left(w_{3}\right) \cup\left(w_{2}, w_{3}\right) \cup\left(w_{1}, w_{2}\right) \cup\left(u_{1}^{1}(G), w_{1}\right)$ and let $G^{\prime \prime}\left(V^{\prime \prime}, E^{\prime \prime}\right)$ be the subgraph of $G$ inside or on the border of cycle $B_{2} \cup P_{2}\left(w_{y}\right) \cup R\left(w_{3}\right) \cup\left(w_{2}, w_{3}\right) \cup\left(w_{1}, w_{2}\right) \cup\left(v_{1}^{1}(G), w_{1}\right)$. Let $f^{\prime}(z)=1$, for each vertex $z$ that is inside or on the border of $C$, let $f^{\prime}(z)=2$, for 
each vertex $z$ that is in $G^{\prime}$, that is in $G\left[F_{1}\right]$, and that is neither inside nor on the border of $C$, and let $f^{\prime}(z)=f(z)+1$, for each vertex $z$ that is in $G^{\prime}$ and that is in $G\left[F_{i}\right]$, for every $i \geq 2$. Let $f^{\prime \prime}\left(w_{1}\right)=1$, let $f^{\prime \prime}\left(w_{2}\right)=1$, let $f^{\prime \prime}(z)=2$, for each vertex $z$ that is in $G^{\prime \prime}$, that is in $G\left[F_{1}\right]$, and that is different from $w_{1}$ and $w_{2}$, and let $f^{\prime \prime}(z)=f(z)+1$, for each vertex $z$ that is $G^{\prime \prime}$ and that is in $G\left[F_{i}\right]$, for every $i \geq 2$. Finally, let $g^{\prime}=\left(w_{1}, w_{2}\right)$ and $g^{\prime \prime}=\left(w_{1}, w_{2}\right)$. If $R\left(w_{3}\right)$ shares vertices with $B_{1}$ (the case in which it shares vertices with $B_{2}$ being analogous), let $G^{\prime}\left(V^{\prime}, E^{\prime}\right)$ be the subgraph of $G$ inside or on the border of cycle $\left(B_{1} \backslash R\left(w_{3}\right)\right) \cup\left(R\left(w_{3}\right) \backslash B_{1}\right) \cup\left(w_{2}, w_{3}\right) \cup\left(w_{1}, w_{2}\right) \cup\left(u_{1}^{1}(G), w_{1}\right)$ and let $G^{\prime \prime}\left(V^{\prime \prime}, E^{\prime \prime}\right)$ be the subgraph of $G$ inside or on the border of cycle $B_{2} \cup P \cup R\left(w_{3}\right) \cup\left(w_{2}, w_{3}\right) \cup\left(w_{1}, w_{2}\right) \cup$ $\left(v_{1}^{1}(G), w_{1}\right)$. Let $f^{\prime}(z)=1$, for each vertex $z$ that is inside or on the border of $C$, let $f^{\prime}(z)=2$, for each vertex $z$ that is in $G^{\prime}$, that is in $G\left[F_{1}\right]$, and that is neither inside nor on the border of $C$, and let $f^{\prime}(z)=f(z)+1$, for each vertex $z$ that is in $G^{\prime}$ and that is in $G\left[F_{i}\right]$, for every $i \geq 2$. Let $f^{\prime \prime}\left(w_{1}\right)=1$, let $f^{\prime \prime}\left(w_{2}\right)=1$, let $f^{\prime \prime}(z)=2$, for each vertex $z$ that is in $G^{\prime \prime}$, that is in $G\left[F_{1}\right]$, and that is different from $w_{1}$ and $w_{2}$, and let $f^{\prime \prime}(z)=f(z)+1$, for each vertex $z$ that is $G^{\prime \prime}$ and that is in $G\left[F_{i}\right]$, for every $i \geq 2$. Finally, let $g^{\prime}=\left(w_{1}, w_{2}\right)$ and $g^{\prime \prime}=\left(w_{1}, w_{2}\right)$.

Lemma $11\left(G^{\prime}, f^{\prime}, g^{\prime}\right)$ and $\left(G^{\prime \prime}, f^{\prime \prime}, g^{\prime \prime}\right)$ are floored graphs.

Proof: $\left(G^{\prime}, f^{\prime}, g^{\prime}\right)$ satisfies Condition $\mathrm{C} 1$, since $G^{\prime}\left[F_{1}^{\prime}\right]$ is a level-2-connected graph (by construction and by Lemma 2) and $g^{\prime}=\left(w_{1}, w_{2}\right)$ is an edge on the outer face of $G^{\prime}$, where $G^{\prime}$ is to the left of $g^{\prime}$ when traversing such an edge from $w_{1}$ to $w_{2}$. $\left(G^{\prime \prime}, f^{\prime \prime}, g^{\prime \prime}\right)$ satisfies Condition $\mathrm{C} 1$, since $G^{\prime \prime}\left[F_{1}^{\prime \prime}\right]$ is an edge (namely such an edge is $\left(w_{1}, w_{2}\right)$ ) and $G^{\prime \prime}$ is to the left of $g^{\prime \prime}$ when traversing such an edge from $w_{2}$ to $w_{1}$.

We prove that $\left(G^{\prime}, f^{\prime}, g^{\prime}\right)$ satisfies Conditions $\mathrm{C} 2-\mathrm{C} 6$, the proof that $\left(G^{\prime \prime}, f^{\prime \prime}, g^{\prime \prime}\right)$ satisfies Conditions $\mathrm{C} 2-\mathrm{C} 6$ being analogous.

For every $i \geq 3$, Conditions C2(i)-(vii) can be shown to be satisfied by the $i$-th floor of $\left(G^{\prime}, f^{\prime}, g^{\prime}\right)$ exactly as in the proof that Conditions $\mathrm{C} 2(\mathrm{i})-($ vii) are satisfied by the $i$-th floor of $\left(G^{\prime}, f^{\prime}, g^{\prime}\right)$ when Case 2 has been applied (see Lemma 8). We prove that the second floor $G^{\prime}\left[F_{2}^{\prime}\right]$ of $\left(G^{\prime}, f^{\prime}, g^{\prime}\right)$ satisfies Conditions C2(i)-(vii). Consider the subgraph $H$ of $G^{\prime}$ composed of $G^{\prime}\left[F_{1}^{\prime}\right]$, of $G^{\prime}\left[F_{2}^{\prime}\right]$, and of edges $\left(w_{1}, u_{1}^{1}(G)\right)$ and $\left(w_{2}, w_{3}\right)$ (see Fig. 7). Denote by $h$ the only internal face of $H$ with more than three incident vertices. Denote by $u_{1}^{2}\left(G^{\prime}\right)=u_{1}^{1}(G), u_{2}^{2}\left(G^{\prime}\right), \ldots, u_{l}^{2}\left(G^{\prime}\right)=w_{3}$ the vertices encountered when traversing the cycle delimiting $h$ from $u_{1}^{1}(G)$ to $w_{3}$ leaving $h$ to the right. Observe that edge $\left(u_{i}^{2}\left(G^{\prime}\right), u_{i+1}^{2}\left(G^{\prime}\right)\right)$ exists, for each $i=1, \ldots, l-1$. If edge $\left(u_{i}^{2}\left(G^{\prime}\right), u_{i+1}^{2}\left(G^{\prime}\right)\right)$ is a chord of $G\left[F_{1}\right]$, then let $U_{i}^{2}$ and $V_{i}^{2}$ be the vertex sets of the two connected components of $G\left[F_{1}\right]$ which are obtained by removing $u_{i}^{2}\left(G^{\prime}\right), u_{i+1}^{2}\left(G^{\prime}\right)$, and their incident edges, where $G\left[U_{i}^{2} \cup\left\{u_{i}^{2}\left(G^{\prime}\right), u_{i+1}^{2}\left(G^{\prime}\right)\right\}\right]$ is to the left of $\left(u_{i}^{2}\left(G^{\prime}\right), u_{i+1}^{2}\left(G^{\prime}\right)\right)$ when traversing such an edge from $u_{i}^{2}\left(G^{\prime}\right)$ to $u_{i+1}^{2}\left(G^{\prime}\right)$. If edge $\left(u_{i}^{2}\left(G^{\prime}\right), u_{i+1}^{2}\left(G^{\prime}\right)\right)$ is not a chord of $G\left[F_{1}\right]$, then such an edge is on the outer face of $G\left[F_{1}\right]$, and let $U_{i}^{2}=\emptyset$. Observe that each of graphs $G_{1}^{\prime 2}=G\left[U_{1}^{2} \cup\left\{u_{1}^{2}\left(G^{\prime}\right), u_{2}^{2}\left(G^{\prime}\right)\right\}\right], G^{\prime 2}=G\left[U_{2}^{2} \cup\left\{u_{2}^{2}\left(G^{\prime}\right), u_{3}^{2}\left(G^{\prime}\right)\right\}\right], \ldots, G^{\prime 2}{ }_{x^{\prime}(2)=l-1}=G\left[U_{l-1}^{2} \cup\right.$ $\left.\left\{u_{l-1}^{2}\left(G^{\prime}\right), u_{l}^{2}\left(G^{\prime}\right)\right\}\right]$ is either a single edge (if the corresponding edge $\left(u_{i}^{2}\left(G^{\prime}\right), u_{i+1}^{2}\left(G^{\prime}\right)\right)$ is not a chord of $G\left[F_{1}\right]$ ) or a level-2-connected graph (if $\left(u_{i}^{2}\left(G^{\prime}\right), u_{i+1}^{2}\left(G^{\prime}\right)\right)$ is a chord of $G\left[F_{1}\right]$, by Lemma 3). Further, by construction and by the fact that $G\left[F_{1}\right]$ is level-2-connected, the sequence $G_{1}^{\prime 2}, G_{2}^{\prime 2}, \ldots, G^{\prime 2}{ }_{x^{\prime}(2)}$ satisfies Conditions $\mathrm{C} 2(\mathrm{i})-($ vii).

If $\left(G^{\prime}, f^{\prime}, g^{\prime}\right)$ has more than two floors, then Condition C3 can be shown to be satisfied by the last floor of $\left(G^{\prime}, f^{\prime}, g^{\prime}\right)$ analogously as in the proof that Conditions C2(i)-(vii) are 


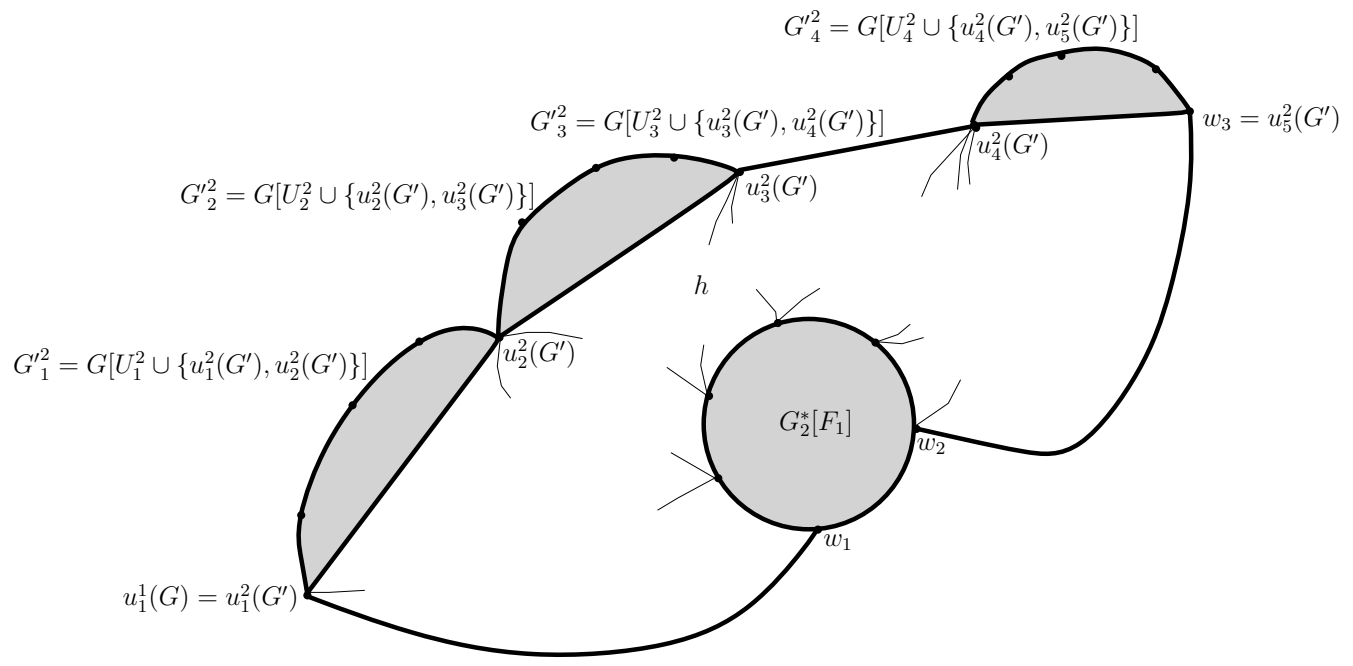

Figure 7: The second floor $G^{\prime}\left[F_{2}^{\prime}\right]$ of $\left(G^{\prime}, f^{\prime}, g^{\prime}\right)$ satisfies Conditions C2(i)-(vii). In this example $U_{3}^{2}=\emptyset$ and $x^{\prime}(2)=4$.

satisfied by the $i$-th floor of $\left(G^{\prime}, f^{\prime}, g^{\prime}\right)$ when Case 2 has been applied (see Lemma 8). If $\left(G^{\prime}, f^{\prime}, g^{\prime}\right)$ has exactly two floors, then Condition C3 can be shown to be satisfied by the second floor of $\left(G^{\prime}, f^{\prime}, g^{\prime}\right)$ analogously as in the above proof that Conditions C2(i)-(vii) are satisfied by the second floor of $\left(G^{\prime}, f^{\prime}, g^{\prime}\right)$.

Condition $\mathrm{C} 4$ can be shown to be satisfied by $\left(G^{\prime}, f^{\prime}, g^{\prime}\right)$ as follows. If an edge exists connecting the $i_{1}$-th floor and the $i_{2}$-th floor of $\left(G^{\prime}, f^{\prime}, g^{\prime}\right)$, with $i_{1}, i_{2} \geq 3$ and $i_{2} \neq$ $i_{1}-1, i_{1}+1$, then such an edge also connects the $\left(i_{1}-1\right)$-th floor and the $\left(i_{2}-1\right)$-th floor of $(G, f, g)$, as $f(z)=f^{\prime}(z)-1$, for each vertex $z$ that is in $G^{\prime}$ and that is in $G^{\prime}\left[F_{i}^{\prime}\right]$, for every $i \geq 3$; however, this is not possible since Condition $\mathrm{C} 4$ is satisfied by $(G, f, g)$. If an edge exists connecting the second floor and the $i_{2}$-th floor of $\left(G^{\prime}, f^{\prime}, g^{\prime}\right)$, with $i_{2} \geq 4$, then such an edge also connects the first floor and the $\left(i_{2}-1\right)$-th floor of $(G, f, g)$, as $f(z)=1$, for each vertex $z$ that is in the second floor of $\left(G^{\prime}, f^{\prime}, g^{\prime}\right)$, and $f(z)=f^{\prime}(z)-1$, for each vertex $z$ that is in $G^{\prime}$ and that is in $G^{\prime}\left[F_{i}^{\prime}\right]$, for every $i \geq 3$; however, this is not possible since Condition $\mathrm{C} 4$ is satisfied by $(G, f, g)$. If an edge exists connecting the first floor and the $i_{2}$-th floor of $\left(G^{\prime}, f^{\prime}, g^{\prime}\right)$, with $i_{2} \geq 3$, then, such an edge would cross the cycle delimiting the outer face of $G\left[F_{1}\right]$, since the first floor of $\left(G^{\prime}, f^{\prime}, g^{\prime}\right)$ is composed of vertices in the $j$-th level of $G\left[F_{1}\right]$, for $j \geq 2$, since $f(z)=f^{\prime}(z)-1$, for each vertex $z$ that is in $G^{\prime}$ and that is in $G^{\prime}\left[F_{i}^{\prime}\right]$, for every $i \geq 3$, and since $(G, f, g)$ satisfies Condition C5; however, this is not possible because of the planarity of $G$.

Condition C5 can be shown to be satisfied by $\left(G^{\prime}, f^{\prime}, g^{\prime}\right)$ as follows. Any floor of $\left(G^{\prime}, f^{\prime}, g^{\prime}\right)$ is a subgraph of a floor of $(G, f, g)$. Further, if $i \geq 3$, then exactly one floor of $\left(G^{\prime}, f^{\prime}, g^{\prime}\right)$ is a subgraph of the $i$-th floor of $(G, f, g)$. It follows that, since $(G, f, g)$ satisfies Condition C5, if at least one of $i$ and $j$ is greater than two, then the $i$-th floor of $\left(G^{\prime}, f^{\prime}, g^{\prime}\right)$ lies in the outer face of the $j$-th floor of $\left(G^{\prime}, f^{\prime}, g^{\prime}\right)$ and vice versa. It remains to show that the first floor of $\left(G^{\prime}, f^{\prime}, g^{\prime}\right)$ lies in the outer face of the second floor of $\left(G^{\prime}, f^{\prime}, g^{\prime}\right)$ and vice versa. By construction, $f^{\prime}(z)=1$ for each vertex $z$ that is inside or on the border of $C$ and for no other vertex of $G^{\prime}$. Hence, if the first floor of $\left(G^{\prime}, f^{\prime}, g^{\prime}\right)$ contains a vertex $z$ of the second floor of $\left(G^{\prime}, f^{\prime}, g^{\prime}\right)$ inside one of its internal faces, then such a vertex lies inside $C$, hence it has $f^{\prime}(z)=1$ and does not belong to the second floor of $\left(G^{\prime}, f^{\prime}, g^{\prime}\right)$, a contradiction. Further, the second floor of $\left(G^{\prime}, f^{\prime}, g^{\prime}\right)$ does not contain any vertex $z$ of the 
first floor of $\left(G^{\prime}, f^{\prime}, g^{\prime}\right)$ inside one of its internal faces, since the first floor of $\left(G^{\prime}, f^{\prime}, g^{\prime}\right)$ induces a 2-connected graph that contains some vertices on the outer face of $G^{\prime}$, hence no vertex of such a graph can be contained inside a cycle of the second floor of $\left(G^{\prime}, f^{\prime}, g^{\prime}\right)$ without violating the planarity of $G$.

If $R\left(w_{3}\right)$ and $B_{1}$ do not share vertices, then Condition C6 is satisfied by $\left(G^{\prime}, f^{\prime}, g^{\prime}\right)$ as $\left(w_{1}, u_{1}^{1}(G)\right) \cup B_{1}$ and $\left(w_{2}, w_{3}\right) \cup R\left(w_{3}\right)$ are the borders of $\left(G^{\prime}, f^{\prime}, g^{\prime}\right)$; otherwise, Condition C6 is satisfied by $\left(G^{\prime}, f^{\prime}, g^{\prime}\right)$ as $\left(w_{1}, u_{1}^{1}(G)\right) \cup\left(B_{1} \backslash R\left(w_{3}\right)\right)$ and $\left(w_{2}, w_{3}\right) \cup R\left(w_{3}\right) \backslash B_{1}$ are the borders of $\left(G^{\prime}, f^{\prime}, g^{\prime}\right)$.

\section{Queue Layouts}

In this section, we show an algorithm for constructing a queue layout of a floored graph $(G, f, g)$. The algorithm splits $(G, f, g)$ into smaller floored graphs using raising paths, recursively constructs queue layouts of such smaller floored graphs, and then combines such layouts to get a queue layout of $(G, f, g)$.

The algorithm receives as an input a floored graph $(G, f, g)$ such that $G$ has $n$ internal vertices and has $k$ floors, and it performs a balanced raising-path decomposition, that is, it repeatedly uses raising paths, according to Cases 1-5 of Section 3, to split $(G, f, g)$ into several floored graphs, each with at most $n / 2$ internal vertices. More precisely, a balanced raising-path decomposition works as follows. Graph $\left(G_{0}^{*}, f_{0}^{*}, g_{0}^{*}\right)=(G, f, g)$ is split into two floored graphs $\left(G_{1}, f_{1}, g_{1}\right)$ and $\left(G_{1}^{*}, f_{1}^{*}, g_{1}^{*}\right)$, where the number of internal vertices of $G_{1}^{*}$ is not less than the number of internal vertices of $G_{1}$, then $\left(G_{1}^{*}, f_{1}^{*}, g_{1}^{*}\right)$ is split into $\left(G_{2}, f_{2}, g_{2}\right)$ and $\left(G_{2}^{*}, f_{2}^{*}, g_{2}^{*}\right)$, where the number of internal vertices of $G_{2}^{*}$ is not less than the number of internal vertices of $G_{2}$, etc., until floored graph $\left(G_{l-1}^{*}, f_{l-1}^{*}, g_{l-1}^{*}\right)$ is split into floored graphs $\left(G_{l}, f_{l}, g_{l}\right)$ and $\left(G_{l}^{*}, f_{l}^{*}, g_{l}^{*}\right)=\left(G_{l+1}, f_{l+1}, g_{l+1}\right)$ such that both $G_{l}$ and $G_{l+1}$ have at most $n / 2$ internal vertices. The split of a graph $\left(G_{j}^{*}, f_{j}^{*}, g_{j}^{*}\right)$ into two floored graphs $\left(G_{j+1}, f_{j+1}, g_{j+1}\right)$ and $\left(G_{j+1}^{*}, f_{j+1}^{*}, g_{j+1}^{*}\right)$ is actually done by applying one of Cases $2-5$. When Case 1 is applied to $\left(G_{j}^{*}, f_{j}^{*}, g_{j}^{*}\right)$, then just one floored graph is obtained, with the same number of internal vertices of $\left(G_{j}^{*}, f_{j}^{*}, g_{j}^{*}\right)$. In such a case, denote still by $\left(G_{j}^{*}, f_{j}^{*}, g_{j}^{*}\right)$ the obtained graph and proceed. Denote by $k\left(G_{j}\right)$ the number of floors of graph $\left(G_{j}, f_{j}, g_{j}\right)$, for $j=1,2, \ldots, l+1$, and by $k\left(G_{j}^{*}\right)$ the number of floors of graph $\left(G_{j}^{*}, f_{j}^{*}, g_{j}^{*}\right)$, for $j=0,1, \ldots, l$.

Before giving more details on the algorithm for constructing a queue layout of $(G, f, g)$, we state the following lemma relating the floors of graphs $\left(G_{j}, f_{j}, g_{j}\right)$ and $\left(G_{j}^{*}, f_{j}^{*}, g_{j}^{*}\right)$ constructed during the balanced raising-path decomposition to the floors and levels of graph $(G, f, g)$.

Lemma 12 There exist a floor $i_{j}$ of $(G, f, g)$ and a floor $p_{j}$ of $\left(G_{j}, f_{j}, g_{j}\right)$ such that (see Fig. 8): (i) for $q=1,2, \ldots, p_{j}$, the $q$-th floor of $\left(G_{j}, f_{j}, g_{j}\right)$ (resp. of $\left(G_{j}^{*}, f_{j}^{*}, g_{j}^{*}\right)$ ) is a graph whose outer face consists of vertices all belonging to the $\left(p_{j}-q+1\right)$-th level of the $i_{j}$-th floor of $(G, f, g)$; (ii) for $q=p_{j}+1, p_{j}+2, \ldots, k\left(G_{j}\right)$ (resp. for $q=p_{j}+1, p_{j}+2, \ldots, k\left(G_{j}^{*}\right)$ ), the $q$-th floor of $\left(G_{j}, f_{j}, g_{j}\right)$ (resp. of $\left(G_{j}^{*}, f_{j}^{*}, g_{j}^{*}\right)$ ) is a graph whose outer face consists of vertices all belonging to the first level of the $\left(i_{j}+q-p_{j}\right)$-th floor of $(G, f, g)$.

Proof: We prove the statement by induction on the index $j$ of graphs $\left(G_{j}, f_{j}, g_{j}\right)$ and $\left(G_{j}^{*}, f_{j}^{*}, g_{j}^{*}\right)$. The statement is indeed true with $i_{0}=1$ and $p_{0}=1$ for $\left(G_{0}^{*}, f_{0}^{*}, g_{0}^{*}\right)$. Suppose 


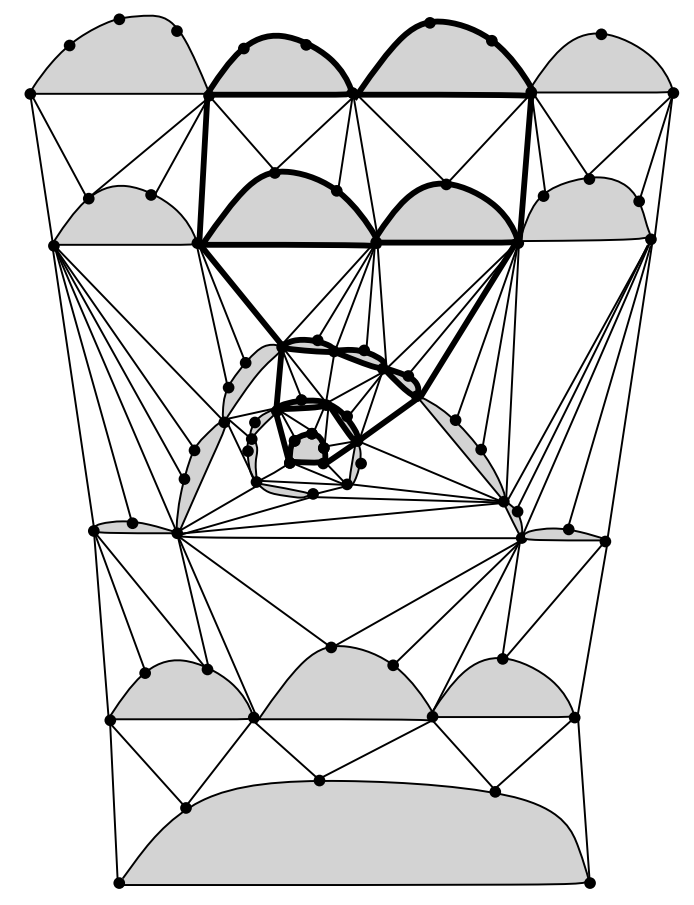

Figure 8: Illustration of the statement of Lemma 12. Graphs $(G, f, g)$ and $\left(G_{j}, f_{j}, g_{j}\right)$ are shown. The borders and the outer faces of the floors of $\left(G_{j}, f_{j}, g_{j}\right)$ are shown by thick lines. In this example $i_{j}=3$ and $p_{j}=3$.

by induction that the statement is satisfied by all graphs $\left(G_{0}^{*}, f_{0}^{*}, g_{0}^{*}\right),\left(G_{1}, f_{1}, g_{1}\right),\left(G_{1}^{*}, f_{1}^{*}, g_{1}^{*}\right)$, $\left(G_{2}, f_{2}, g_{2}\right),\left(G_{2}^{*}, f_{2}^{*}, g_{2}^{*}\right), \ldots,\left(G_{j}, f_{j}, g_{j}\right),\left(G_{j}^{*}, f_{j}^{*}, g_{j}^{*}\right)$. We prove that $\left(G_{j+1}, f_{j+1}, g_{j+1}\right)$ and $\left(G_{j+1}^{*}, f_{j+1}^{*}, g_{j+1}^{*}\right)$ also satisfy the statement of the lemma. Actually, we do the inductive proof only for $\left(G_{j+1}, f_{j+1}, g_{j+1}\right)$, since the proof for $\left(G_{j+1}^{*}, f_{j+1}^{*}, g_{j+1}^{*}\right)$ is analogous.

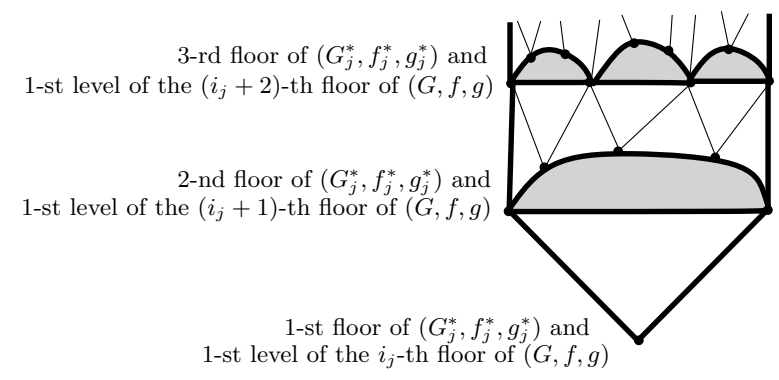

(a)

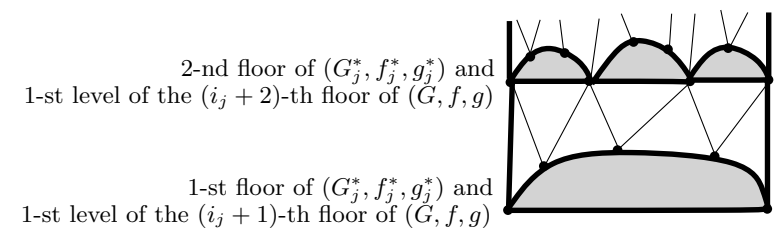

(b)

Figure 9: (a) Previous graph $\left(G_{j}^{*}, f_{j}^{*}, g_{j}^{*}\right)$ and (b) resulting graph $\left(G_{j}^{*}, f_{j}^{*}, g_{j}^{*}\right)$ if Case 1 is applied and the previous $p_{j}$ was equal to one.

If Case 1 is applied to $\left(G_{j}^{*}, f_{j}^{*}, g_{j}^{*}\right)$, then the resulting graph $\left(G_{j}^{*}, f_{j}^{*}, g_{j}^{*}\right)$ is easily shown to satisfy the statement of the lemma. Namely, if the value of $p_{j}$ for the previous $\left(G_{j}^{*}, f_{j}^{*}, g_{j}^{*}\right)$ was equal to one, then set $i_{j}$ equal to one plus the value of $i_{j}$ for the previous $\left(G_{j}^{*}, f_{j}^{*}, g_{j}^{*}\right)$ and set $p_{j}=1$ (see Fig. 9 ); if the value of $p_{j}$ for the previous $\left(G_{j}^{*}, f_{j}^{*}, g_{j}^{*}\right)$ was greater than one, then set $i_{j}$ equal to the value of $i_{j}$ for the previous $\left(G_{j}^{*}, f_{j}^{*}, g_{j}^{*}\right)$ and $p_{j}$ equal to the value of $p_{j}$ for the previous $\left(G_{j}^{*}, f_{j}^{*}, g_{j}^{*}\right)$ minus one (see Fig. 10). 


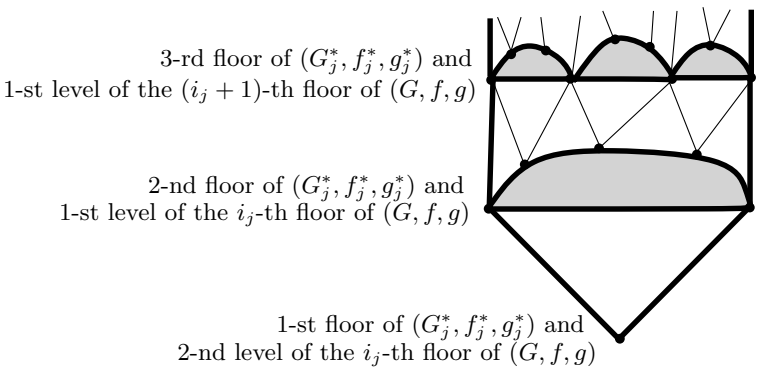

(a)

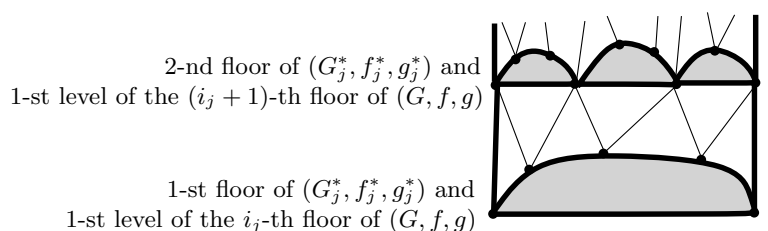

(b)

Figure 10: (a) Previous graph $\left(G_{j}^{*}, f_{j}^{*}, g_{j}^{*}\right)$ and (b) resulting graph $\left(G_{j}^{*}, f_{j}^{*}, g_{j}^{*}\right)$ if Case 1 is applied and the previous $p_{j}$ was greater than one (in this example the previous $p_{j}$ was equal to 2).

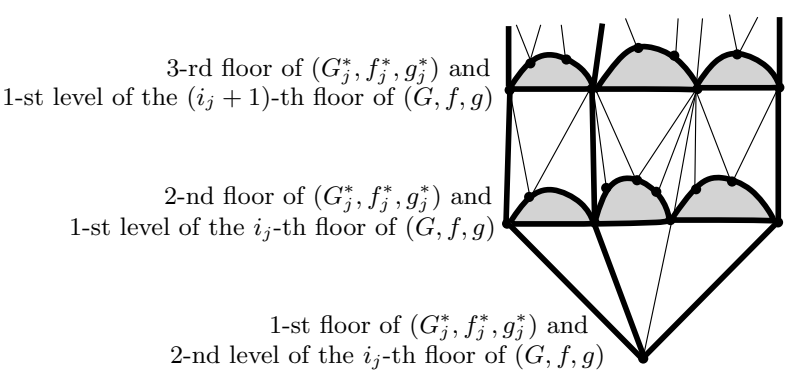

(a)

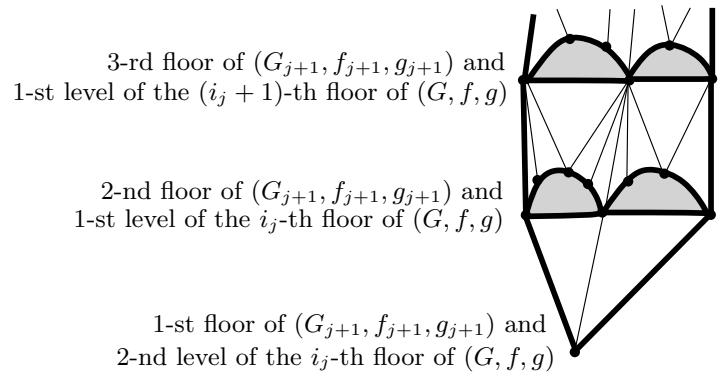

(b)

Figure 11: (a) Graph $\left(G_{j}^{*}, f_{j}^{*}, g_{j}^{*}\right)$ and (b) graph $\left(G_{j+1}, f_{j+1}, g_{j+1}\right)$ if Case 2 is applied. In this example $p_{j}=2$.

If Case 2 is applied to $\left(G_{j}^{*}, f_{j}^{*}, g_{j}^{*}\right)$, then $\left(G_{j+1}, f_{j+1}, g_{j+1}\right)$ satisfies the statement of the lemma with $i_{j+1}=i_{j}$ and with $p_{j+1}=p_{j}$ (see Fig. 11). Namely, since the first floor of $\left(G_{j}^{*}, f_{j}^{*}, g_{j}^{*}\right)$ consists only of vertex $g_{j}^{*}$, by the hypotheses of Case 2 , and since the first floor of $\left(G_{j+1}, f_{j+1}, g_{j+1}\right)$ is also a single vertex $g_{j+1}=g_{j}^{*}$, it follows that the first floor of $\left(G_{j+1}, f_{j+1}, g_{j+1}\right)$ satisfies Property (i) with $i_{j+1}=i_{j}$. Further, since the $q$-th floor of $\left(G_{j}^{*}, f_{j}^{*}, g_{j}^{*}\right)$ satisfies Property (i) with $i_{j}$ if $2 \leq q \leq p_{j}$, since the $q$-th floor of $\left(G_{j}^{*}, f_{j}^{*}, g_{j}^{*}\right)$ satisfies Property (ii) with $i_{j}$ if $p_{j}+1 \leq q \leq k\left(G_{j}\right)$, and since the $q$-th floor of $\left(G_{j+1}, f_{j+1}, g_{j+1}\right)$ is a subsequence (which may possibly degenerate into a single vertex) of the sequence of edges and level-2-connected graphs that compose the $q$-th floor of $\left(G_{j}^{*}, f_{j}^{*}, g_{j}^{*}\right)$, for $q=2,3, \ldots, k\left(G_{j}^{*}\right)$, it follows that $\left(G_{j+1}, f_{j+1}, g_{j+1}\right)$ satisfies Property (i) with $i_{j+1}=i_{j}$ if $2 \leq q \leq p_{j+1}$ and satisfies Property (ii) with $i_{j+1}=i_{j}$ if $p_{j+1}+1 \leq$ $q \leq k\left(G_{j+1}\right)$.

If Case 3 is applied to $\left(G_{j}^{*}, f_{j}^{*}, g_{j}^{*}\right)$, then $\left(G_{j+1}, f_{j+1}, g_{j+1}\right)$ satisfies the statement of the lemma with $i_{j+1}=i_{j}$ and with $p_{j+1}=p_{j}$ (see Fig. 12). Namely, since the first floor of $\left(G_{j}^{*}, f_{j}^{*}, g_{j}^{*}\right)$ consists only of edge $g_{j}^{*}$, by the hypotheses of Case 3 , and since the first floor of $\left(G_{j+1}, f_{j+1}, g_{j+1}\right)$ is a single vertex $g_{j+1}$ that is one end-vertex of $g_{j}^{*}$, it follows that the first floor of $\left(G_{j+1}, f_{j+1}, g_{j+1}\right)$ satisfies Property (i) with $i_{j+1}=i_{j}$. Further, since the $q$-th floor of $\left(G_{j}^{*}, f_{j}^{*}, g_{j}^{*}\right)$ satisfies Property (i) with $i_{j}$ if $2 \leq q \leq p_{j}$, since the $q$-th floor of $\left(G_{j}^{*}, f_{j}^{*}, g_{j}^{*}\right)$ satisfies Property (ii) with $i_{j}$ if $p_{j}+1 \leq q \leq k\left(G_{j}\right)$, and since the $q$-th floor of $\left(G_{j+1}, f_{j+1}, g_{j+1}\right)$ is a subsequence (which may possibly degenerate into a 


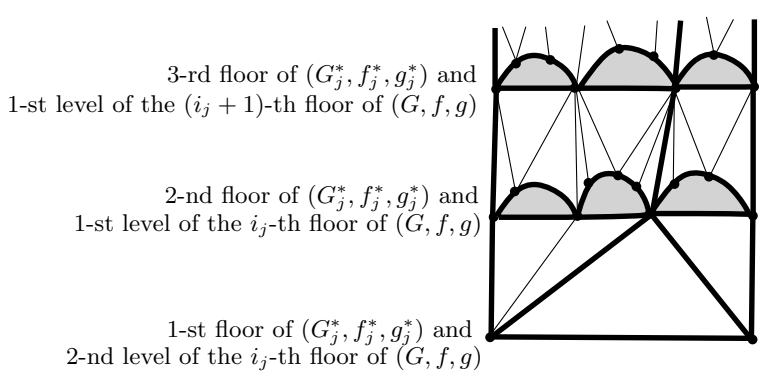

(a)

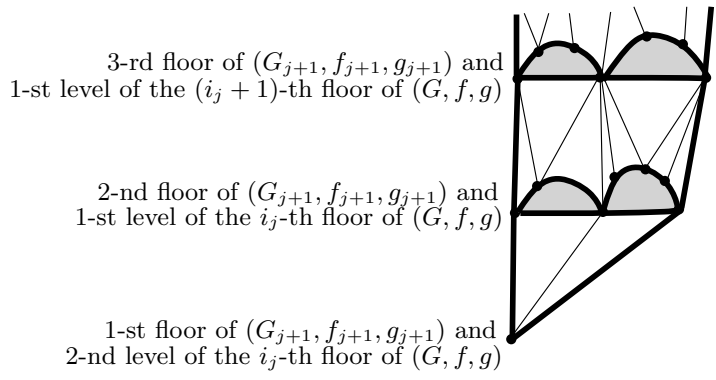

(b)

Figure 12: (a) Graph $\left(G_{j}^{*}, f_{j}^{*}, g_{j}^{*}\right)$ and (b) graph $\left(G_{j+1}, f_{j+1}, g_{j+1}\right)$ if Case 3 is applied. In this example $p_{j}=2$.

single vertex) of the sequence of edges and level-2-connected graphs that compose the $q$-th floor of $\left(G_{j}^{*}, f_{j}^{*}, g_{j}^{*}\right)$, for $q=2,3, \ldots, k\left(G_{j}^{*}\right)$, it follows that $\left(G_{j+1}, f_{j+1}, g_{j+1}\right)$ satisfies Property (i) with $i_{j+1}=i_{j}$ if $2 \leq q \leq p_{j+1}$ and satisfies Property (ii) with $i_{j+1}=i_{j}$ if $p_{j+1}+1 \leq q \leq k\left(G_{j+1}\right)$.

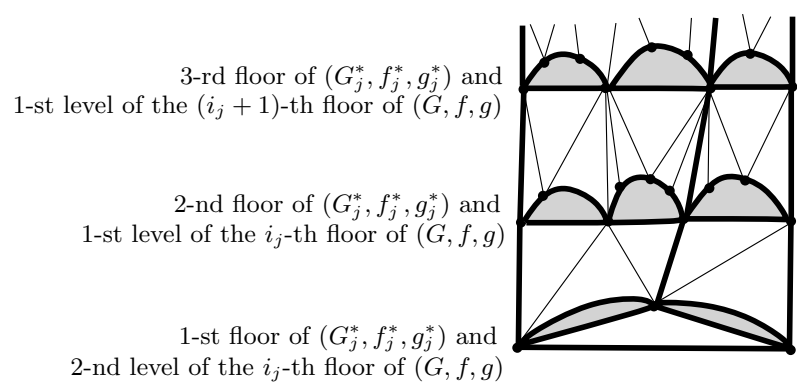

(a)

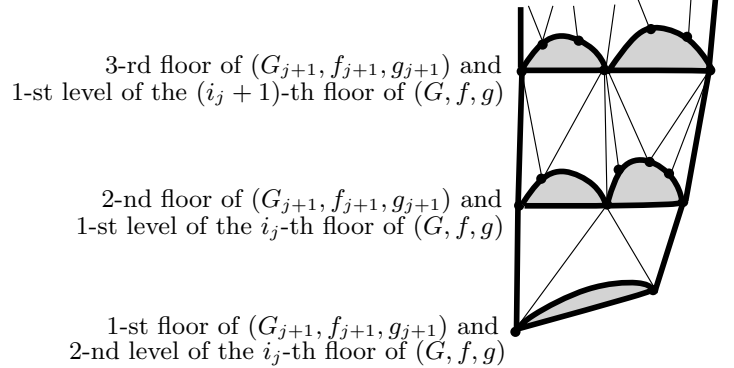

(b)

Figure 13: (a) Graph $\left(G_{j}^{*}, f_{j}^{*}, g_{j}^{*}\right)$ and (b) graph $\left(G_{j+1}, f_{j+1}, g_{j+1}\right)$ if Case 4 is applied. In this example $p_{j}=2$.

If Case 4 is applied to $\left(G_{j}^{*}, f_{j}^{*}, g_{j}^{*}\right)$, then $\left(G_{j+1}, f_{j+1}, g_{j+1}\right)$ satisfies the statement of the lemma with $i_{j+1}=i_{j}$ and with $p_{j+1}=p_{j}$ (see Fig. 13). Namely, since the outer face of the first floor of $\left(G_{j+1}, f_{j+1}, g_{j+1}\right)$ is composed of a set of vertices which is a subset of the set of vertices on the outer face of the first floor of $\left(G_{j}^{*}, f_{j}^{*}, g_{j}^{*}\right)$, by the hypotheses of Case 4 , and since the first floor of $\left(G_{j}^{*}, f_{j}^{*}, g_{j}^{*}\right)$ satisfies Property (i) with $i_{j}$, it follows that the first floor of $\left(G_{j+1}, f_{j+1}, g_{j+1}\right)$ satisfies Property (i) with $i_{j+1}=i_{j}$. Further, since the $q$-th floor of $\left(G_{j}^{*}, f_{j}^{*}, g_{j}^{*}\right)$ satisfies Property (i) with $i_{j}$ if $2 \leq q \leq p_{j}$, since the $q$-th floor of $\left(G_{j}^{*}, f_{j}^{*}, g_{j}^{*}\right)$ satisfies Property (ii) with $i_{j}$ if $p_{j}+1 \leq q \leq k\left(G_{j}\right)$, and since the $q$-th floor of $\left(G_{j+1}, f_{j+1}, g_{j+1}\right)$ is a subsequence (which may possibly degenerate into a single vertex) of the sequence of edges and level-2-connected graphs that compose the $q$-th floor of $\left(G_{j}^{*}, f_{j}^{*}, g_{j}^{*}\right)$, for $q=2,3, \ldots, k\left(G_{j}^{*}\right)$, it follows that $\left(G_{j+1}, f_{j+1}, g_{j+1}\right)$ satisfies Property (i) with $i_{j+1}=i_{j}$ if $2 \leq q \leq p_{j+1}$ and satisfies Property (ii) with $i_{j+1}=i_{j}$ if $p_{j+1}+1 \leq q \leq k\left(G_{j+1}\right)$.

Finally, if Case 5 is applied to $\left(G_{j}^{*}, f_{j}^{*}, g_{j}^{*}\right)$, then $\left(G_{j+1}, f_{j+1}, g_{j+1}\right)$ satisfies the statement of the lemma with $i_{j+1}=i_{j}$ and with $p_{j+1}=p_{j}+1$ (see Fig. 14). Namely, by induction the first floor of $\left(G_{j}^{*}, f_{j}^{*}, g_{j}^{*}\right)$ is a level-2-connected graph whose outer face is composed 


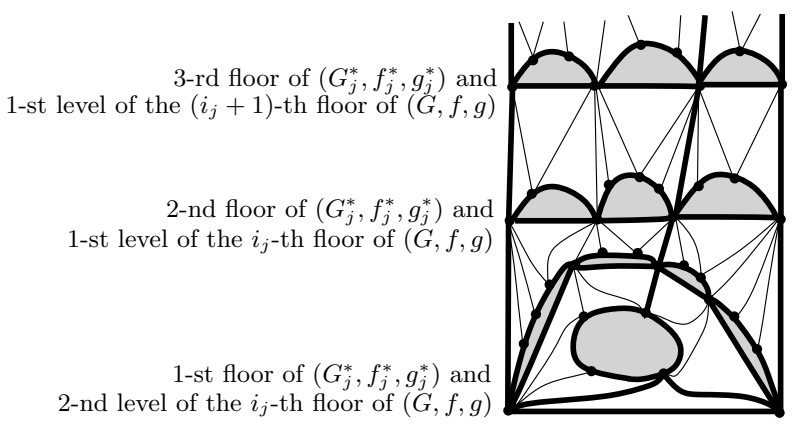

(a)

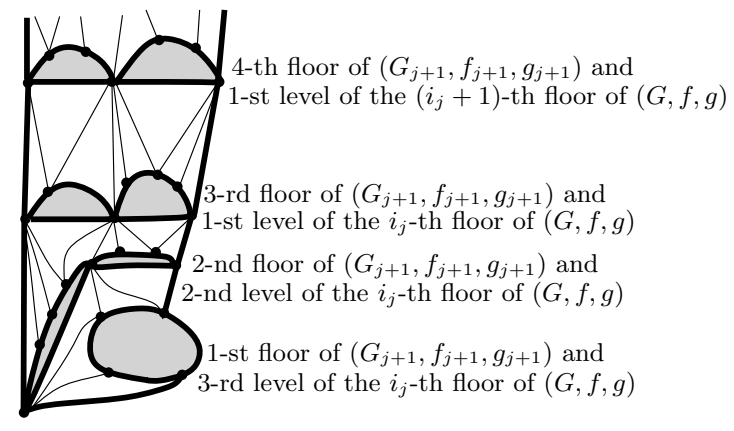

(b)

Figure 14: (a) Graph $\left(G_{j}^{*}, f_{j}^{*}, g_{j}^{*}\right)$ and (b) graph $\left(G_{j+1}, f_{j+1}, g_{j+1}\right)$ if Case 5 is applied. In this example $p_{j}=2$.

of vertices that are on the $p_{j}$-th level of the $i_{j}$-floor of $(G, f, g)$. It follows from the construction of Case 5 that the first floor of $\left(G_{j+1}, f_{j+1}, g_{j+1}\right)$ is either a single edge whose end-vertices are on the $\left(p_{j}+1\right)$-level of the $i_{j}$-floor of $(G, f, g)$, or it is a level-2-connected graph whose outer face is composed of vertices that are on the $\left(p_{j}+1\right)$-level of the $i_{j}$-floor of $(G, f, g)$. Hence, the first floor of $\left(G_{j+1}, f_{j+1}, g_{j+1}\right)$ satisfies Property (i) with $i_{j+1}=i_{j}$. Further, since the $q$-th floor of $\left(G_{j}^{*}, f_{j}^{*}, g_{j}^{*}\right)$ satisfies Property (i) with $i_{j}$ if $1 \leq q \leq p_{j}$, since the $q$-th floor of $\left(G_{j}^{*}, f_{j}^{*}, g_{j}^{*}\right)$ satisfies Property (ii) with $i_{j}$ if $p_{j}+1 \leq q \leq k\left(G_{j}\right)$, and since the $q$-th floor of $\left(G_{j+1}, f_{j+1}, g_{j+1}\right)$ is a subsequence (which may possibly degenerate into a single vertex) of the sequence of edges and level-2-connected graphs that compose the $(q-1)$-th floor of $\left(G_{j}^{*}, f_{j}^{*}, g_{j}^{*}\right)$, for $q=2,3, \ldots, k\left(G_{j+1}\right)$, it follows that $\left(G_{j+1}, f_{j+1}, g_{j+1}\right)$ satisfies Property (i) with $i_{j+1}=i_{j}$ if $2 \leq q \leq p_{j+1}$ and satisfies Property (ii) with $i_{j+1}=i_{j}$ if $p_{j+1}+1 \leq q \leq k\left(G_{j+1}\right)$.

The algorithm to construct a queue layout of $(G, f, g)$ builds an ordered list $L$ of vertices and, at the end of its execution, the order given by $L$ will be the total order of the vertices of $G$. The algorithm maintains two invariants. Invariant $A$ : All the vertices of the $i$-th floor of $(G, f, g)$ come in $L$ before all the vertices of the $(i+1)$-th floor of $(G, f, g)$, for each $1 \leq i \leq k-1$. Invariant $B$ : The border vertices of the $i$-th floor of $(G, f, g)$ come in $L$ before all the non-border vertices of the $i$-th floor of $(G, f, g)$, for each $1 \leq i \leq k$.

Invariant A corresponds to partition $L$ into sublists, where sublist $L_{i}$ contains all and only the vertices of the $i$-th floor of $(G, f, g)$. Hence, the vertices of each floor of $(G, f, g)$ appear consecutively in $L$. Recall that the splits of Cases 1-5 may originate floored graphs whose floors are different from the ones of $(G, f, g)$. However, when a vertex is inserted into $L$, it is inserted in the sublist $L_{i}$ of the floor it belongs to in $(G, f, g)$.

Recall that the $i$-th floor of $(G, f, g)$ is composed of a sequence of edges or level-2connected graphs $G_{j}^{i}$, where such a sequence can degenerate to be a single vertex for the first and/or the last floor. Denote by $m\left(G_{j}^{i}\right)$ the number of levels of $G_{j}^{i}$ (if $G_{j}^{i}$ is an edge, then let $m\left(G_{j}^{i}\right)=1$ ) and denote by $m(i)$ the maximum among the $m\left(G_{j}^{i}\right)$, for $j=$ $1, \ldots, x(i)$. If the $i$-th level is a vertex, then let $m(i)=1$. For each $i=1,2, \ldots, k$, partition $L_{i}$ into consecutive sublists $L_{i, 1}, L_{i, 2}, \ldots, L_{i, m(i)}$. Each list $L_{i, j}$ is in turn partitioned into two consecutive sublists $L_{i, j}^{\prime}$ and $L_{i, j}^{\prime \prime}$. See Fig. 15.

We now sketch the algorithm for constructing $L$. It starts by placing, for each $1 \leq$ 

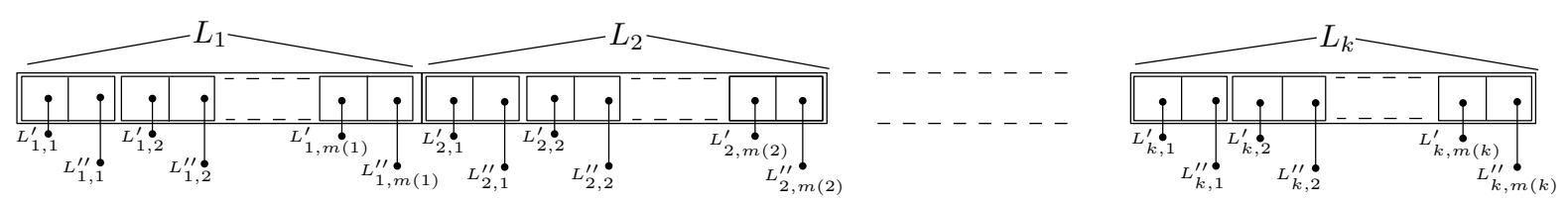

Figure 15: The partition of $L$ into sublists.

$p \leq k$, the border vertices on the $p$-th floor of $(G, f, g)$ at the first positions of $L_{p, 1}^{\prime}$ so that Invariant $\mathrm{B}$ is satisfied. Then, the graphs $\left(G_{j}, f_{j}, g_{j}\right)$ obtained by the balanced raising-path decomposition are processed one at a time. When $\left(G_{j}, f_{j}, g_{j}\right)$ is processed, an ordering $J$ of the vertices of $G_{j}$ is recursively constructed. The ordering of the vertices of $G_{j}$ in $L$ is almost the same as in $J$. Namely, each floor of $\left(G_{j}, f_{j}, g_{j}\right)$ has in $L$ the same vertex ordering as in $J$ and the vertices of each floor of $\left(G_{j}, f_{j}, g_{j}\right)$ appear consecutively in $L$ (except for the border vertices of $\left(G_{j}, f_{j}, g_{j}\right)$ on such a floor). However, the order of the floors of $\left(G_{j}, f_{j}, g_{j}\right)$ in $L$ may differ from the order of the floors of $\left(G_{j}, f_{j}, g_{j}\right)$ in $J$. Namely, while the floors of $\left(G_{j}, f_{j}, g_{j}\right)$ are ordered in $J$ according to the definition of floored graph, such floors are ordered in $L$ according to the level of the floor of $(G, f, g)$ their outer faces belong to. Then, there exists an index $p_{j}$ such that the ordering of the floors of $\left(G_{j}, f_{j}, g_{j}\right)$ in $L$ is the $p_{j}$-th first, then the $\left(p_{j}-1\right)$-th, then the $\left(p_{j}-2\right)$-th, $\ldots$, then the first, then the $\left(p_{j}+1\right)$, then the $\left(p_{j}+2\right)$-th, $\ldots$, then the last. We now formally state the algorithm.

\section{Algorithm Vertex-Ordering}

For $p=1,2, \ldots, k$, insert vertex $u_{1}^{p}(G)$ into $L_{p, 1}^{\prime}$. For $p=1,2, \ldots, k$, if vertex $v_{x(p)}^{p}(G)$ does not belong to $L$, then append it to $L_{p, 1}^{\prime}$.

Construct graphs $\left(G_{1}, f_{1}, g_{1}\right),\left(G_{2}, f_{2}, g_{2}\right), \ldots,\left(G_{l+1}, f_{l+1}, g_{l+1}\right)$ performing a balanced raising-path decomposition of $(G, f, g)$. For $i=1,2, \ldots, l+1$, process graph $\left(G_{j}, f_{j}, g_{j}\right)$ :

- For $y=1,2, \ldots, k\left(G_{j}\right)$, consider border vertices $u_{1}^{y}\left(G_{j}\right)$ and $v_{x(y)}^{y}\left(G_{j}\right)$ of $\left(G_{j}, f_{j}, g_{j}\right)$.

- If $u_{1}^{y}\left(G_{j}\right)$ does not belong to $L$, let $p$ be the floor of $u_{1}^{y}\left(G_{j}\right)$ in $(G, f, g)$ and let $q$ be the level of $u_{1}^{y}\left(G_{j}\right)$ in $G\left[F_{p}\right]$. Append $u_{1}^{y}\left(G_{j}\right)$ to $L_{p, q}^{\prime}$.

- If $v_{x(y)}^{y}\left(G_{j}\right)$ does not belong to $L$, let $p$ be the floor of $v_{x(y)}^{y}\left(G_{j}\right)$ in $(G, f, g)$ and let $q$ be the level of $v_{x(y)}^{y}\left(G_{j}\right)$ in $G\left[F_{p}\right]$. Append $v_{x(y)}^{y}\left(G_{j}\right)$ to $L_{p, q}^{\prime}$.

- Recursively construct a vertex ordering $J$ of $\left(G_{j}, f_{j}, g_{j}\right)$. By Lemma 12 , for each $1 \leq r \leq k\left(G_{j}\right)$, all the vertices on the outer face of the $r$-th floor of $\left(G_{j}, f_{j}, g_{j}\right)$ are on the $q$-th level of the $p$-th floor of $\left(G_{j}, f_{j}, g_{j}\right)$, for some $p$ and $q$ depending on $r$. Append all the non-border vertices of the $r$-th floor of $\left(G_{j}, f_{j}, g_{j}\right)$ to $L_{p, q}^{\prime \prime}$ in the same order as they appear in $J$.

Observe that, for $j=1,2, \ldots, l+1$, the algorithm first inserts into $L$ the border vertices of $\left(G_{j}, f_{j}, g_{j}\right)$ and then inserts into $L$ the non-border vertices of $\left(G_{j}, f_{j}, g_{j}\right)$. Further, when the algorithm processes $\left(G_{j}, f_{j}, g_{j}\right)$, all the border vertices of $\left(G_{j-1}, f_{j-1}, g_{j-1}\right)$ have been already inserted into $L$.

The following lemma relates the order of the vertices in list $L_{p, q}^{\prime}$ to the order in which the vertices are inserted by Algorithm Vertex-Ordering. For any two vertices $a$ and $b$, we write $a \prec b$ if $a$ comes before $b$ in $L$. 
Lemma 13 Let $a$ and $b$ be two vertices in $L_{p, q}^{\prime}$ such that $a \prec b$. Then, a is inserted into $L_{p, q}^{\prime}$ before $b$.

Proof: The proof easily descends from the fact that, when a vertex is inserted into a list $L_{p, q}^{\prime}$, it is appended to such a list.

We now study the edges of $(G, f, g)$ relating the end-vertices of such edges to their position in $L$ and to the floor of $(G, f, g)$ they belong to. A visible edge is an edge of $G$ that has one end-vertex in a list $L_{p, q}^{\prime}$, for some $p$ and $q$, and one end-vertex in a list $L_{r, s}^{\prime}$, for some $r$ and s. A semi-visible edge is an edge of $G$ that has one end-vertex in a list $L_{p, q}^{\prime}$, for some $p$ and $q$, and one end-vertex in a list $L_{r, s}^{\prime \prime}$, for some $r$ and $s$. An invisible edge is an edge of $G$ that has one end-vertex in a list $L_{p, q}^{\prime \prime}$, for some $p$ and $q$, and one end-vertex in a list $L_{r, s}^{\prime \prime}$, for some $r$ and $s$. Intuitively, visible, semi-visible, and invisible edges are such that both end-vertices, one end-vertex, and no end-vertex, respectively, belong to the borders of graphs $\left(G_{j}, f_{j}, g_{j}\right)$. The inter-floor edges are the edges of $G$ that connect vertices on consecutive floors of $(G, f, g)$. The intra-floor edges are the edges of $G$ that connect vertices on the same floor of $(G, f, g)$.

Lemma 14 Every edge of $G$ is either a visible edge, or a semi-visible edge, or an invisible edge. $L$.

Proof: The proof easily descends from the fact that the lists $L_{p, q}^{\prime}$ and $L_{p, q}^{\prime \prime}$ partition

Lemma 15 Every edge of $G$ is either an intra-floor edge or a inter-floor edge.

Proof: The proof easily descends from the fact that $(G, f, g)$ satisfies Condition C4.

Lemma 16 Every inter-floor edge of $(G, f, g)$ is an inter-floor edge of a graph $\left(G_{j}, f_{j}, g_{j}\right)$, for some $1 \leq j \leq l+1$.

Proof: By Lemma 12, each floor of $\left(G_{j}, f_{j}, g_{j}\right)$ is a subgraph of a floor of $(G, f, g)$. Hence, an intra-floor edge of $\left(G_{j}, f_{j}, g_{j}\right)$ is also an intra-floor edge of $(G, f, g)$. It remains to show that every inter-floor edge of $(G, f, g)$ belongs to a graph $\left(G_{j}, f_{j}, g_{j}\right)$, for some $1 \leq j \leq l+1$. An easy inductive proof shows that every edge of $(G, f, g)$ belongs to a graph $\left(G_{j}, f_{j}, g_{j}\right)$, for some $1 \leq j \leq l+1$, except for edge $g_{j}^{*}$ of $\left(G_{j}^{*}, f_{j}^{*}, g_{j}^{*}\right)$, for each $0 \leq j \leq l-1$. However, by definition of floored graphs, $g_{j}^{*}$ is an edge between two vertices on the same floor of $\left(G_{j}^{*}, f_{j}^{*}, g_{j}^{*}\right)$. By Lemma 12, such two vertices belong to the $i_{j}$-th floor of $(G, f, g)$, for some $i_{j}$. It follows that $g_{j}^{*}$ is an intra-floor edge of $(G, f, g)$ and the lemma follows.

Lemma 17 Let $(a, b)$ be an inter-floor visible edge such that a belongs to the $i$-th floor of $(G, f, g)$ and $b$ belongs to the $(i+1)$-th floor of $(G, f, g)$. Then, $a$ is in $L_{i, 1}^{\prime}$ and $b$ is in $L_{i+1,1}^{\prime}$. 
Proof: By planarity and by the fact that $(G, f, g)$ satisfies Condition C5, $a$ is in the first level of $G\left[F_{i}\right]$ and $b$ is in the first level of $G\left[F_{i+1}\right]$. Since $(a, b)$ is visible, $a$ and $b$ are in some $L_{p, q}^{\prime}$ and $L_{r, s}^{\prime}$, respectively. Since $a$ belongs to the $i$-th floor of $(G, f, g)$ and $b$ belongs to the $(i+1)$-th floor of $(G, f, g), p=i$ and $r=i+1$. Since $a$ is in the first level of $G\left[F_{i}\right]$ and $b$ is in the first level of $G\left[F_{i+1}\right], q=1$ and $s=1$.

Lemma 18 Let $(a, b)$ be an inter-floor semi-visible edge such that a belongs to the $i$-th floor of $(G, f, g)$ and $b$ belongs to the $(i+1)$-th floor of $(G, f, g)$. Then, either $a$ is in $L_{i, 1}^{\prime}$ and $b$ is in $L_{i+1,1}^{\prime \prime}$ or $a$ is in $L_{i, 1}^{\prime \prime}$ and $b$ is in $L_{i+1,1}^{\prime}$.

Proof: By planarity and by the fact that $(G, f, g)$ satisfies Condition C5, $a$ is in the first level of $G\left[F_{i}\right]$ and $b$ is in the first level of $G\left[F_{i+1}\right]$. Since $(a, b)$ is semi-visible, either $a$ is in some $L_{p, q}^{\prime}$ and $b$ is in some $L_{r, s}^{\prime \prime}$, or $a$ is in some $L_{p, q}^{\prime \prime}$ and $b$ is in some $L_{r, s}^{\prime}$. Since $a$ belongs to the $i$-th floor of $(G, f, g)$ and $b$ belongs to the $(i+1)$-th floor of $(G, f, g), p=i$ and $r=i+1$. Since $a$ is in the first level of $G\left[F_{i}\right]$ and $b$ is in the first level of $G\left[F_{i+1}\right]$, $q=1$ and $s=1$.

Lemma 19 Let $(a, b)$ be an inter-floor invisible edge such that a belongs to the $i$-th floor of $(G, f, g)$ and $b$ belongs to the $(i+1)$-th floor of $(G, f, g)$. Then, $a$ is in $L_{i, 1}^{\prime \prime}$ and $b$ is in $L_{i+1,1}^{\prime \prime}$.

Proof: By planarity and by the fact that $(G, f, g)$ satisfies Condition C5, $a$ is in the first level of $G\left[F_{i}\right]$ and $b$ is in the first level of $G\left[F_{i+1}\right]$. Since $(a, b)$ is invisible, $a$ and $b$ are in some $L_{p, q}^{\prime \prime}$ and $L_{r, s}^{\prime \prime}$, respectively. Since $a$ belongs to the $i$-th floor of $(G, f, g)$ and $b$ belongs to the $(i+1)$-th floor of $(G, f, g), p=i$ and $r=i+1$. Since $a$ is in the first level of $G\left[F_{i}\right]$ and $b$ is in the first level of $G\left[F_{i+1}\right], q=1$ and $s=1$.

Lemma 20 Let $(a, b)$ be an intra-floor visible edge such that $a$ and $b$ belong to the $i$-th floor of $(G, f, g)$. Then, either $a$ and $b$ are both in the same list $L_{i, q}^{\prime}$, or $a$ is in $L_{i, q}^{\prime}$ and $b$ is in $L_{i, q+1}^{\prime}$, or a is in $L_{i, q}^{\prime}$ and $b$ is in $L_{i, q-1}^{\prime}$, for some $q$.

Proof: By definition of intra-floor edge, $a$ and $b$ are in lists $L_{i, q}^{\prime}$ and $L_{i, r}^{\prime}$, for some $q$ and $r$. We prove that $r$ is equal either to $q-1$, or to $q$, or to $q+1$. Suppose, for a contradiction, that $r>q+1$. As the vertices in $L_{i, q}^{\prime}$ (resp. in $L_{i, r}^{\prime}$ ) are the border vertices of some graph $\left(G_{j}, f_{j}, g_{j}\right)$ and belong to the $q$-th level (resp. to the $r$-th level) of the $i$-th floor of $(G, f, g)$, then edge $(a, b)$ crosses the graph induced by the $(q+1)$-th level of the $i$-th floor of $(G, f, g)$, thus contradicting the planarity of $G$. It can be analogously proved that $r<q-1$ does not hold, and the lemma follows.

Lemma 21 Let $(a, b)$ be an intra-floor semi-visible edge such that $a$ and $b$ belong to the $i$-th floor of $(G, f, g)$. Then, either $a$ is in $L_{i, q}^{\prime}$ and $b$ is in $L_{i, q}^{\prime \prime}$, or a is in $L_{i, q}^{\prime \prime}$ and $b$ is in $L_{i, q}^{\prime}$, or a is in $L_{i, q}^{\prime}$ and $b$ is in $L_{i, q+1}^{\prime \prime}$, or a is in $L_{i, q}^{\prime \prime}$ and $b$ is in $L_{i, q+1}^{\prime}$, or a is in $L_{i, q}^{\prime}$ and $b$ is in $L_{i, q-1}^{\prime \prime}$, or a is in $L_{i, q}^{\prime \prime}$ and $b$ is in $L_{i, q-1}^{\prime}$, for some $q$.

Proof: By definition of intra-floor edge, $a$ and $b$ are respectively in lists $L_{i, q}^{\prime}$ and $L_{i, r}^{\prime \prime}$, for some $q$ and $r$, or $a$ and $b$ are respectively in lists $L_{i, q}^{\prime \prime}$ and $L_{i, r}^{\prime}$, for some $q$ and $r$. Suppose that $a$ and $b$ are respectively in lists $L_{i, q}^{\prime}$ and $L_{i, r}^{\prime \prime}$, for some $q$ and $r$, the other 
case being analogous. We prove that $r$ is either equal to $q-1$, or to $q$, or to $q+1$. Suppose, for a contradiction, that $r>q+1$. The vertices in $L_{i, q}^{\prime}$ are the border vertices of some graph $\left(G_{j}, f_{j}, g_{j}\right)$ and belong to the $q$-th level of the $i$-th floor of $(G, f, g)$. Further, by Lemma 12 the vertices in $L_{i, q}^{\prime \prime}$ are the non-border vertices of a floor of $\left(G_{j}, f_{j}, g_{j}\right)$; the outer face of such a floor has all incident vertices belonging to the $r$-th level of the $i$-th floor of $(G, f, g)$. It follows that edge $(a, b)$ crosses the graph induced by the $(q+1)$-th level of the $i$-th floor of $(G, f, g)$, thus contradicting the planarity of $G$. It can be analogously proved that $r<q-1$ does not hold, and the lemma follows.

Lemma 22 Let $(a, b)$ be an intra-floor invisible edge such that $a$ and $b$ belong to the $i$-th floor of $(G, f, g)$. Then, either $a$ is in $L_{i, q}^{\prime \prime}$ and $b$ is in $L_{i, q}^{\prime \prime}$, or $a$ is in $L_{i, q}^{\prime \prime}$ and $b$ is in $L_{i, q+1}^{\prime \prime}$, or $a$ is in $L_{i, q}^{\prime \prime}$ and $b$ is in $L_{i, q-1}^{\prime \prime}$, for some $q$.

Proof: Since $(a, b)$ is an intra-floor edge, $a$ belongs to a list $L_{i, q}^{\prime \prime}$ and $b$ belongs to a list $L_{i, r}^{\prime \prime}$, for some $q$ and $r$. Suppose, for a contradiction, that $r>q+1$. Then, since $b$ belongs to a graph whose outer face is composed of vertices on the $r$-th level of the $i$-floor of $(G, f, g)$, it follows that $(a, b)$ crosses the subgraph of $G$ induced by the vertices on the $(q+1)$-th level of the $p$-th floor of $(G, f, g)$, thus contradicting the planarity of $G$. It can be analogously proved that $r<q-1$ does not hold and the lemma follows.

We now state some basic properties of Algorithm Vertex-Ordering. In particular, such properties deal with the vertices inserted into $L$ for each floor of a graph $\left(G_{j}, f_{j}, g_{j}\right)$ once it is processed by the algorithm, and deal with the edges such vertices share with vertices that were in $L$ before processing $\left(G_{j}, f_{j}, g_{j}\right)$.

Lemma 23 When Algorithm Vertex-Ordering processes a graph $\left(G_{j}, f_{j}, g_{j}\right)$, it inserts at most one vertex into each list $L^{\prime}(p, q)$, with the exception of the case in which Case 5 is applied to split $\left(G_{j-1}^{*}, f_{j-1}^{*}, g_{j-1}^{*}\right)$ into two graphs $\left(G_{j}, f_{j}, g_{j}\right)$ and $\left(G_{j}^{*}, f_{j}^{*}, g_{j}^{*}\right)$. In this case, two vertices (those on the first floor of $\left(G_{j}, f_{j}, g_{j}\right)$ ) are inserted into one list $L^{\prime}(p, q)$ and at most one vertex is inserted into every other list $L^{\prime}(p, q)$.

Proof: When Algorithm Vertex-Ordering processes a graph $\left(G_{j}, f_{j}, g_{j}\right)$ obtained from $\left(G_{j-1}^{*}, f_{j-1}^{*}, g_{j-1}^{*}\right)$ by applying one of Cases $1-4$, by construction it inserts into $L$ at most one border vertex for each floor of $\left(G_{j}, f_{j}, g_{j}\right)$ (as all the border vertices of $\left(G_{j-1}^{*}, f_{j-1}^{*}, g_{j-1}^{*}\right)$ have been already inserted into $L$ when $\left(G_{j}, f_{j}, g_{j}\right)$ is processed and as $\left(G_{j}, f_{j}, g_{j}\right)$ satisfies Condition C6). By Lemma 12 , at most one floor of $\left(G_{j-1}^{*}, f_{j-1}^{*}, g_{j-1}^{*}\right)$ has the outer face (and hence the border vertices) belonging to the $q$-th level of the $p$-th floor of $(G, f, g)$, hence at most one vertex is inserted into $L^{\prime}(p, q)$, for each $p$ and $q$. When Algorithm Vertex-Ordering processes a graph $\left(G_{j}, f_{j}, g_{j}\right)$ obtained from $\left(G_{j-1}^{*}, f_{j-1}^{*}, g_{j-1}^{*}\right)$ by applying Case 5 , by construction it inserts into $L$ at most one border vertex for each floor of $\left(G_{j}, f_{j}, g_{j}\right)$ (as all the border vertices of $\left(G_{j-1}^{*}, f_{j-1}^{*}, g_{j-1}^{*}\right)$ have been already inserted into $L$ when $\left(G_{j}, f_{j}, g_{j}\right)$ is processed and as $\left(G_{j}, f_{j}, g_{j}\right)$ satisfies Condition C6), with the exception of the first floor of $\left(G_{j}, f_{j}, g_{j}\right)$, for which two vertices are inserted. By Lemma 12, at most one floor of $\left(G_{j-1}^{*}, f_{j-1}^{*}, g_{j-1}^{*}\right)$ has the outer face (and hence the border vertices) belonging to the $q$-th level of the $p$-th floor of $(G, f, g)$. Hence, at most one vertex is inserted into $L^{\prime}(p, q)$, for each $p$ and $q$, except for the $p$ and $q$ such that the first floor of $\left(G_{j-1}^{*}, f_{j-1}^{*}, g_{j-1}^{*}\right)$ has the outer face belonging to the $q$-th level of the $p$-th floor of $(G, f, g)$. For such $p$ and $q$ the two border vertices on the first floor of $\left(G_{j}, f_{j}, g_{j}\right)$ are inserted into $L^{\prime}(p, q)$. 
Lemma 24 Suppose that, when Algorithm Vertex-Ordering processes a graph $\left(G_{j}, f_{j}, g_{j}\right)$, it inserts one vertex a into a list $L^{\prime}(p, q)$. Then, at most one vertex $b \neq a$ that is in $L^{\prime}(p, q)$ before $\left(G_{j}, f_{j}, g_{j}\right)$ is processed has adjacent vertices that: (i) are not in $L^{\prime}(p, q)$ after $\left(G_{j}, f_{j}, g_{j}\right)$ has been processed and (ii) belong to graphs $\left(G_{j+1}, f_{j+1}, g_{j+1}\right), \ldots,\left(G_{l+1}, f_{l+1}, g_{l+1}\right)$.

Proof: By Lemma 12, at most one floor of $\left(G_{j-1}^{*}, f_{j-1}^{*}, g_{j-1}^{*}\right)$ has the outer face (and hence the border vertices) belonging to the $q$-th level of the $p$-th floor of $(G, f, g)$. Hence, at most two vertices $b$ and $c$ that are in $\left(G_{j-1}^{*}, f_{j-1}^{*}, g_{j-1}^{*}\right)$ and that have been inserted into $L^{\prime}(p, q)$ before $\left(G_{j}, f_{j}, g_{j}\right)$ is processed have neighbors that: (i) are not in $L^{\prime}(p, q)$ after $\left(G_{j}, f_{j}, g_{j}\right)$ has been processed and (ii) belong to graphs $\left(G_{j+1}, f_{j+1}, g_{j+1}\right), \ldots,\left(G_{l+1}, f_{l+1}, g_{l+1}\right)$. By planarity, any vertex that does not belong to the border of $\left(G_{j-1}^{*}, f_{j-1}^{*}, g_{j-1}^{*}\right)$ has no neighbors that: (i) are not in $L^{\prime}(p, q)$ after $\left(G_{j}, f_{j}, g_{j}\right)$ has been processed by Algorithm Vertex-Ordering and (ii) belong to graphs $\left(G_{j+1}, f_{j+1}, g_{j+1}\right), \ldots,\left(G_{l+1}, f_{l+1}, g_{l+1}\right)$. Further, since $a$ is inserted into $L^{\prime}(p, q)$, such a vertex belongs to $q$-th level of the $p$-th floor of $(G, f, g)$. It follows that $a$ belongs to a raising path that has no common vertex with the borders of $\left(G_{j-1}^{*}, f_{j-1}^{*}, g_{j-1}^{*}\right)$ in the floors of $\left(G_{j-1}^{*}, f_{j-1}^{*}, g_{j-1}^{*}\right)$ that precede the floor of $\left(G_{j-1}^{*}, f_{j-1}^{*}, g_{j-1}^{*}\right) a, b$, and $c$ belong to. Hence, when $\left(G_{j-1}^{*}, f_{j-1}^{*}, g_{j-1}^{*}\right)$ is split into two graphs $\left(G_{j}, f_{j}, g_{j}\right)$ and $\left(G_{j}^{*}, f_{j}^{*}, g_{j}^{*}\right)$, one of $b$ and $c$, say $b$, belongs to the border of $\left(G_{j}, f_{j}, g_{j}\right)$ and the other one, say $c$, to the border of $\left(G_{j}^{*}, f_{j}^{*}, g_{j}^{*}\right)$. By planarity, only $c$ can have neighbors that: (i) are not in $L^{\prime}(p, q)$ after $\left(G_{j}, f_{j}, g_{j}\right)$ has been processed by Algorithm Vertex-Ordering and (ii) belong to graphs $\left(G_{j+1}, f_{j+1}, g_{j+1}\right), \ldots,\left(G_{l+1}, f_{l+1}, g_{l+1}\right)$. The lemma follows.

The following lemmata discuss "how many" inter-floor and intra-floor edges are inserted during the processing of $\left(G_{j}, f_{j}, g_{j}\right)$. Edges are also classified according to their visibility.

Lemma 25 Let a be a vertex that is inserted into list $L^{\prime}(p, 1)$ when graph $\left(G_{j}, f_{j}, g_{j}\right)$ is processed by Algorithm Vertex-Ordering. Then, after $\left(G_{j}, f_{j}, g_{j}\right)$ has been processed, $L^{\prime}(p+1,1)$ contains at most three neighbors of a. Further, if such neighbors are three, then one of them has been inserted into $L^{\prime}(p+1,1)$ during the processing of $\left(G_{j}, f_{j}, g_{j}\right)$.

Proof: First, we prove that $a$ has at most two neighbors that are into $L^{\prime}(p+1,1)$ and that belong to the border of $\left(G_{j-1}^{*}, f_{j-1}^{*}, g_{j-1}^{*}\right)$. Since every floor of a floored graph contains at most two border vertices, vertex $a$ can be adjacent to at most two border vertices on the same floor of $\left(G_{j-1}^{*}, f_{j-1}^{*}, g_{j-1}^{*}\right)$. By Lemma 12 , at most one floor of $\left(G_{j-1}^{*}, f_{j-1}^{*}, g_{j-1}^{*}\right)$ has the outer face (and hence the border vertices) belonging to the first level of the $(p+1)$-th floor of $(G, f, g)$, hence $a$ has at most two neighbors that are in $L^{\prime}(p+1,1)$ and that are border vertices of $\left(G_{j-1}^{*}, f_{j-1}^{*}, g_{j-1}^{*}\right)$. Second, if $a$ is adjacent to a border vertex $b$ that is on first level of the $(p+1)$-th floor of $(G, f, g)$, that is not on the border of $\left(G_{j-1}^{*}, f_{j-1}^{*}, g_{j-1}^{*}\right)$, and that is already in $L^{\prime}(p+1,1)$ when $a$ is inserted into $L^{\prime}(p, 1)$, then edge $(a, b)$ would cause a crossing, thus violating the planarity of $G$. Third, at most one neighbor of $a$ is inserted into $L^{\prime}(p+1,1)$ when $\left(G_{j}, f_{j}, g_{j}\right)$ is processed. Namely, by Lemma 23, at most one vertex is inserted into each list $L^{\prime}(r, s)$ when $\left(G_{j}, f_{j}, g_{j}\right)$ is processed, with the exception of when Case 5 is applied to split $\left(G_{j-1}^{*}, f_{j-1}^{*}, g_{j-1}^{*}\right)$. Namely, in such a case the two vertices on the first floor of $\left(G_{j}, f_{j}, g_{j}\right)$ are inserted into the same list $L^{\prime}(r, s)$. However, such vertices do not belong to the first level of any floor of $(G, f, g)$, hence they are not in $L^{\prime}(p+1,1)$. 
Lemma 26 Let a be a vertex that is inserted into list $L^{\prime}(p, 1)$ when graph $\left(G_{j}, f_{j}, g_{j}\right)$ is processed by Algorithm Vertex-Ordering. Then, after $\left(G_{j}, f_{j}, g_{j}\right)$ has been processed, $L^{\prime}(p-1,1)$ contains at most three neighbors of a. Further, if such neighbors are three, then one of them has been inserted into $L^{\prime}(p-1,1)$ during the processing of $\left(G_{j}, f_{j}, g_{j}\right)$.

Proof: Analogous to the proof of Lemma 25.

Lemma 27 Let $N(j, p)$ be the set of non-border vertices of $\left(G_{j}, f_{j}, g_{j}\right)$ inserted into $L_{p, 1}^{\prime \prime}$, for every $2 \leq p \leq k$. Then, there exist at most two vertices of $L_{p-1,1}^{\prime}$ that are connected to the vertices in $N(j, p)$. Further, such two vertices are the border vertices of $\left(G_{j}, f_{j}, g_{j}\right)$ that belong to the first level of the $(p-1)$-th floor of $(G, f, g)$.

Proof: Since $L_{p-1,1}^{\prime}$ contains all and only the border vertices of graphs $\left(G_{1}, f_{1}, g_{1}\right), \ldots$, $\left(G_{l+1}, f_{l+1}, g_{l+1}\right)$ that belong to the first level of the $p$-th floor of $(G, f, g)$, by planarity the only vertices belonging to $L_{p-1,1}^{\prime}$ that are possibly adjacent to vertices in $N(j, p)$ are the border vertices of $\left(G_{j}, f_{j}, g_{j}\right)$ that belong to the first level of the $(p-1)$-th floor of $(G, f, g)$. Since, by Lemma 12 , at most one floor of $\left(G_{j}, f_{j}, g_{j}\right)$ has the outer face (and hence the border vertices) belonging to the first level of the $(p-1)$-th floor of $(G, f, g)$, the lemma follows.

Lemma 28 Let $N(j, p)$ be the set of non-border vertices of $\left(G_{j}, f_{j}, g_{j}\right)$ inserted into $L_{p, 1}^{\prime \prime}$, for every $1 \leq p \leq k-1$. Then, there exist at most two vertices of $L_{p+1,1}^{\prime}$ that are connected to the vertices in $N(j, p)$. Further, such two vertices are the border vertices of $\left(G_{j}, f_{j}, g_{j}\right)$ that belong to the first level of the $(p+1)$-th floor of $(G, f, g)$.

Proof: Analogous to the proof of Lemma 27.

Lemma 29 Let a be a vertex that is inserted into list $L^{\prime}(p, q)$ when graph $\left(G_{j}, f_{j}, g_{j}\right)$ is processed by Algorithm Vertex-Ordering. Then, after graph $\left(G_{j}, f_{j}, g_{j}\right)$ has been processed, $L^{\prime}(p, q)$ contains at most two neighbors of a.

Proof: First, we prove that $a$ has at most two neighbors that are into $L^{\prime}(p, q)$ and that belong to the border of $\left(G_{j-1}^{*}, f_{j-1}^{*}, g_{j-1}^{*}\right)$. As every floor of a floored graph contains at most two border vertices, vertex $a$ can be adjacent to at most two border vertices on the same floor of $\left(G_{j-1}^{*}, f_{j-1}^{*}, g_{j-1}^{*}\right)$. By Lemma 12 , at most one floor of $\left(G_{j-1}^{*}, f_{j-1}^{*}, g_{j-1}^{*}\right)$ has the outer face (and hence the border vertices) belonging to the $q$-th level of the $p$-th floor of $(G, f, g)$, hence $a$ has at most two neighbors that are in $L^{\prime}(p, q)$ and that are border vertices of $\left(G_{j-1}^{*}, f_{j-1}^{*}, g_{j-1}^{*}\right)$. Second, if $a$ is adjacent to a border vertex $b$ that is on $q$-th level of the $p$-th floor of $(G, f, g)$, that is not on the border of $\left(G_{j-1}^{*}, f_{j-1}^{*}, g_{j-1}^{*}\right)$, and that is already in $L^{\prime}(p, q)$ when $a$ is inserted into $L^{\prime}(p, q)$, then edge $(a, b)$ would cause a crossing, thus violating the planarity of $G$. Third, either no neighbor of $a$ is inserted into $L^{\prime}(p, q)$ when $\left(G_{j}, f_{j}, g_{j}\right)$ is processed, or $a$ has at most one neighbor in $L^{\prime}(p, q)$ after processing $\left(G_{j}, f_{j}, g_{j}\right)$. Namely, by Lemma 23 , at most one vertex is inserted into $L^{\prime}(p, q)$ when $\left(G_{j}, f_{j}, g_{j}\right)$ is processed, with the exception of when Case 5 is applied to split $\left(G_{j-1}^{*}, f_{j-1}^{*}, g_{j-1}^{*}\right)$. Hence, if the two vertices on the first floor of $\left(G_{j}, f_{j}, g_{j}\right)$ are not inserted into $L^{\prime}(p, q)$, then no neighbor of $a$ is inserted into $L^{\prime}(p, q)$ when $\left(G_{j}, f_{j}, g_{j}\right)$ is processed. Otherwise, the two vertices on the first floor of $\left(G_{j}, f_{j}, g_{j}\right)$ are inserted 
into $L^{\prime}(p, q)$ and $a$ is one of such vertices (as it is inserted into $L^{\prime}(p, q)$ when processing $\left.\left(G_{j}, f_{j}, g_{j}\right)\right)$. However, in such a case no border vertex of $\left(G_{j-1}^{*}, f_{j-1}^{*}, g_{j-1}^{*}\right)$ belongs to the $q$-th level of the $p$-th floor of $(G, f, g)$ hence $a$ has at most one neighbor in $L^{\prime}(p, q)$.

Lemma 30 Let a be a vertex that is inserted into a list $L^{\prime}(p, q)$ when a graph $\left(G_{j}, f_{j}, g_{j}\right)$ is processed by Algorithm Vertex-Ordering. Suppose that $q>1$. Then, after graph $\left(G_{j}, f_{j}, g_{j}\right)$ has been processed by Algorithm Vertex-Ordering, there exist at most three neighbors of a in $L^{\prime}(p, q-1)$. Further, if there are exactly three neighbors of a in $L^{\prime}(p, q-1)$, then one of them has been inserted when $\left(G_{j}, f_{j}, g_{j}\right)$ has been processed by Algorithm Vertex-Ordering.

Proof: By the construction of Algorithm Vertex-Ordering, the only vertices inserted into $L^{\prime}(p, q)$ are the vertices belonging to the $q$-th level of the $p$-th floor of $(G, f, g)$ that are part of the border of some graph $\left(G_{x}, f_{x}, g_{x}\right)$, for $1 \leq x \leq l+1$. Graph $\left(G_{j}, f_{j}, g_{j}\right)$ is obtained by splitting a graph $\left(G_{j-1}^{*}, f_{j-1}^{*}, g_{j-1}^{*}\right)$ into two graphs $\left(G_{j}, f_{j}, g_{j}\right)$ and $\left(G_{j-1}^{*}, f_{j-1}^{*}, g_{j-1}^{*}\right)$. As every floor of a floored graph contains at most two border vertices, vertex $a$ can be adjacent to at most two border vertices on the same floor of $\left(G_{j-1}^{*}, f_{j-1}^{*}, g_{j-1}^{*}\right)$. By Lemma 12 , at most one floor of $\left(G_{j-1}^{*}, f_{j-1}^{*}, g_{j-1}^{*}\right)$ has the outer face (and hence the border vertices) belonging to the $(q-1)$-th level of the $p$-th floor of $(G, f, g)$, hence $a$ has at most two neighbors that are in $L^{\prime}(p, q-1)$ and that are border vertices of $\left(G_{j-1}^{*}, f_{j-1}^{*}, g_{j-1}^{*}\right)$. If $a$ is adjacent to a border vertex $b$ that is on the $(q-1)$-th level of the $p$-th floor of $(G, f, g)$, that is not on the border of $\left(G_{j-1}^{*}, f_{j-1}^{*}, g_{j-1}^{*}\right)$, and that is already in $L^{\prime}(p, q-1)$ when $a$ is inserted into $L^{\prime}(p, q)$, then edge $(a, b)$ would cause a crossing, thus violating the planarity of $G$. It remains to prove that at most one neighbor of $a$ is inserted into $L^{\prime}(p, q-1)$ when $\left(G_{j}, f_{j}, g_{j}\right)$ is processed. In each of Cases $1-5$, at most one vertex is inserted for each floor of $\left(G_{j}, f_{j}, g_{j}\right)$, with the exception of the first floor of $\left(G_{j}, f_{j}, g_{j}\right)$ when Case 5 is applied to split $\left(G_{j-1}^{*}, f_{j-1}^{*}, g_{j-1}^{*}\right)$. Namely, in such a case two vertices are inserted on the first floor of $\left(G_{j}, f_{j}, g_{j}\right)$. However, such vertices are not in $L^{\prime}(p, q-1)$, as otherwise Lemma 12 would be contradicted.

Lemma 31 Let a be a vertex that is inserted into a list $L^{\prime}(p, q)$ when a graph $\left(G_{j}, f_{j}, g_{j}\right)$ is processed by Algorithm Vertex-Ordering. Suppose that $q<m(p)$. Then, after graph $\left(G_{j}, f_{j}, g_{j}\right)$ has been processed by Algorithm Vertex-Ordering, there exist at most three neighbors of a in $L^{\prime}(p, q+1)$. Further, if there are exactly three neighbors of a in $L^{\prime}(p, q+1)$, then one of them has been inserted when $\left(G_{j}, f_{j}, g_{j}\right)$ has been processed by Algorithm Vertex-Ordering.

Proof: By the construction of Algorithm Vertex-Ordering, the only vertices inserted into $L^{\prime}(p, q)$ are the vertices belonging to the $q$-th level of the $p$-th floor of $(G, f, g)$ that are part of the border of some graph $\left(G_{x}, f_{x}, g_{x}\right)$, for $1 \leq x \leq l+1$. Graph $\left(G_{j}, f_{j}, g_{j}\right)$ is obtained by splitting a graph $\left(G_{j-1}^{*}, f_{j-1}^{*}, g_{j-1}^{*}\right)$ into two graphs $\left(G_{j}, f_{j}, g_{j}\right)$ and $\left(G_{j-1}^{*}, f_{j-1}^{*}, g_{j-1}^{*}\right)$. As every floor of a floored graph contains at most two border vertices, vertex $a$ can be adjacent to at most two border vertices on the same floor of $\left(G_{j-1}^{*}, f_{j-1}^{*}, g_{j-1}^{*}\right)$. By Lemma 12 , at most one floor of $\left(G_{j-1}^{*}, f_{j-1}^{*}, g_{j-1}^{*}\right)$ has the outer face (and hence the border vertices) belonging to the $(q+1)$-th level of the $p$-th floor of $(G, f, g)$, hence $a$ has at most two neighbors that are in $L^{\prime}(p, q+1)$ and that are border vertices of $\left(G_{j-1}^{*}, f_{j-1}^{*}, g_{j-1}^{*}\right)$. If $a$ is adjacent to a border vertex $b$ that is on the $(q+1)$-th level of the $p$-th floor of $(G, f, g)$, that is not on the border of $\left(G_{j-1}^{*}, f_{j-1}^{*}, g_{j-1}^{*}\right)$, and that 
is already in $L^{\prime}(p, q+1)$ when $a$ is inserted into $L^{\prime}(p, q)$, then edge $(a, b)$ would cause a crossing, thus violating the planarity of $G$. It remains to prove that at most one neighbor of $a$ is inserted into $L^{\prime}(p, q+1)$ when $\left(G_{j}, f_{j}, g_{j}\right)$ is processed. In each of Cases $1-5$, at most one vertex is inserted for each floor of $\left(G_{j}, f_{j}, g_{j}\right)$, with the exception of the first floor of $\left(G_{j}, f_{j}, g_{j}\right)$ when Case 5 is applied to split $\left(G_{j-1}^{*}, f_{j-1}^{*}, g_{j-1}^{*}\right)$. Namely, in such a case two vertices are inserted on the first floor of $\left(G_{j}, f_{j}, g_{j}\right)$. However, if the first floor of $\left(G_{j}, f_{j}, g_{j}\right)$ has the outer face whose vertices belong to the $(q+1)$-th level of the $p$-th floor of $(G, f, g)$, then, by Lemma 12 , no border vertex of $\left(G_{j-1}^{*}, f_{j-1}^{*}, g_{j-1}^{*}\right)$ is on the $(q+1)$-th level of the $p$-th floor of $(G, f, g)$. Hence, if $a$ is adjacent to the vertices of the first floor of $\left(G_{j}, f_{j}, g_{j}\right)$ and such vertices belong to the $(q+1)$-th level of the $p$-th floor of $(G, f, g)$, then $a$ has no other neighbor in $L^{\prime}(p, q+1)$.

Lemma 32 Let $N(j, p, q)$ be the set of non-border vertices of $\left(G_{j}, f_{j}, g_{j}\right)$ inserted into $L_{p, q}^{\prime \prime}$, for every $1 \leq p \leq k$ and every $1 \leq q \leq k\left(G_{j}\right)$. Then, there exist at most two vertices of $L_{p, q}^{\prime}$ that are connected to the vertices in $N(j, p, q)$. Further, such two vertices are the border vertices of $\left(G_{j}, f_{j}, g_{j}\right)$ that belong to the q-th level of the $p$-th floor of $(G, f, g)$.

Proof: Since $L_{p, q}^{\prime}$ contains all and only the border vertices of graphs $\left(G_{1}, f_{1}, g_{1}\right), \ldots$, $\left(G_{l+1}, f_{l+1}, g_{l+1}\right)$ that belong to the $q$-th level of the $p$-th floor of $(G, f, g)$, by planarity the only vertices belonging to $L_{p, q}^{\prime}$ that are possibly adjacent to vertices in $N(j, p, q)$ are the border vertices of $\left(G_{j}, f_{j}, g_{j}\right)$ that belong to the $q$-th level of the $p$-th floor of $(G, f, g)$. Since, by Lemma 12, at most one floor of $\left(G_{j}, f_{j}, g_{j}\right)$ has the outer face (and hence the border vertices) belonging to the $q$-th level of the $p$-th floor of $(G, f, g)$, the lemma follows.

Lemma 33 Let $N(j, p, q)$ be the set of non-border vertices of $\left(G_{j}, f_{j}, g_{j}\right)$ inserted into $L_{p, q}^{\prime \prime}$, for every $1 \leq p \leq k$ and every $2 \leq q \leq k\left(G_{j}\right)$. Then, there exist at most two vertices of $L_{p, q-1}^{\prime}$ that are connected to the vertices in $N(j, p, q)$. Further, such two vertices are the border vertices of $\left(G_{j}, f_{j}, g_{j}\right)$ that belong to the $(q-1)$-th level of the $p$-th floor of $(G, f, g)$.

Proof: Analogous to the proof of Lemma 32.

Lemma 34 Let $N(j, p, q)$ be the set of non-border vertices of $\left(G_{j}, f_{j}, g_{j}\right)$ inserted into $L_{p, q}^{\prime \prime}$, for every $1 \leq p \leq k$ and every $1 \leq q \leq k\left(G_{j}\right)-1$. Then, there exist at most two vertices of $L_{p, q+1}^{\prime}$ that are connected to the vertices in $N(j, p, q)$. Further, such two vertices are the border vertices of $\left(G_{j}, f_{j}, g_{j}\right)$ that belong to the $(q+1)$-th level of the $p$-th floor of $(G, f, g)$.

Proof: Analogous to the proof of Lemma 32.

We now introduce some definitions and notation to compute the queue number of $G$ once the vertices of $G$ have the order specified by $L$. Let $q(G, f, g), q_{\text {intra }}(G, f, g)$, and $q_{\text {inter }}(G, f, g)$ denote the number of queues needed to embed all the edges, only the intra-floor edges, and only the inter-floor edges, respectively, of a floored graph $(G, f, g)$ once the vertices of $G$ have the order computed by Algorithm Vertex-Ordering. Let $q(n)$, $q_{\text {intra }}(n)$, and $q_{\text {inter }}(n)$ denote the maximum of $q(G, f, g), q_{\text {intra }}(G, f, g)$, and $q_{\text {inter }}(G, f, g)$, respectively, over all possible graphs $(G, f, g)$ with $n$ non-border vertices. 
Let $G$ be a graph and let $\prec$ be a vertex ordering of $G$. A set of edges $\left(a_{1}, b_{1}\right),\left(a_{2}, b_{2}\right), \ldots$, $\left(a_{m}, b_{m}\right)$ is a rainbow of size $m$ if $a_{1} \prec a_{2} \prec \cdots \prec a_{m} \prec b_{m} \prec \cdots \prec b_{2} \prec b_{1}$. We use the following results.

Lemma 35 [24] The queue number of a graph $G$ is the minimum, taken among all vertex orderings $\prec$ of $G$, of the maximum size of a rainbow in $\prec$.

Lemma 36 Let $G(A \cup B, E)$, be a bipartite graph. Consider a vertex ordering $\prec$ of $A \cup B$ such that $a \prec b$ whenever $a \in A$ and $b \in B$ and let $Q$ be the maximum size of a rainbow in $\prec$. Consider a vertex ordering $\prec^{\prime}$ of $A \cup B$ such that $b \prec a$ whenever $a \in A$ and $b \in B$, such that $a_{1} \prec^{\prime} a_{2}$ whenever $a_{1}, a_{2} \in A$ and $a_{1} \prec a_{2}$, and such that $b_{1} \prec^{\prime} b_{2}$ whenever $b_{1}, b_{2} \in B$ and $b_{1} \prec b_{2}$. Then, the maximum size of a rainbow in $\prec^{\prime}$ is $Q$.

Proof: Consider any two edges $\left(a_{1}, b_{1}\right)$ and $\left(a_{2}, b_{2}\right)$ that do not nest in $\prec$. Suppose, w.l.o.g., that $a_{1} \prec a_{2} \prec b_{1} \prec b_{2}$. Then, $b_{1} \prec^{\prime} b_{2} \prec^{\prime} a_{1} \prec^{\prime} a_{2}$, hence edges $\left(a_{1}, b_{1}\right)$ and $\left(a_{2}, b_{2}\right)$ do not nest in $\prec^{\prime}$, as well, and the lemma follows.

We now prove that the edges of $(G, f, g)$ can be embedded into $O\left(\log ^{2} n\right)$ queues, once the vertices of $G$ have the order of $L$.

Lemma 37 (1) The size of a rainbow of visible inter-floor edges of $(G, f, g)$ is at most five. (2) The size of a rainbow of semi-visible inter-floor edges of $(G, f, g)$ is at most eight. (3) The size of a rainbow of invisible inter-floor edges of $(G, f, g)$ is at most the maximum size of a rainbow of inter-floor edges in one of graphs $\left(G_{1}, f_{1}, g_{1}\right), \ldots,\left(G_{l+1}, f_{l+1}, g_{l+1}\right)$.

Proof: (Proof of Statement (1)). If $f\left(a_{1}\right)=p_{1}, f\left(a_{2}\right)=p_{1}+1, f\left(b_{1}\right)=p_{2}>p_{1}$, and $f\left(b_{2}\right)=p_{2}+1$, then inter-floor edges $\left(a_{1}, a_{2}\right)$ and $\left(b_{1}, b_{2}\right)$ do not nest, as either $a_{1} \prec a_{2} \prec b_{1} \prec b_{2}$ or $a_{1} \prec b_{1} \prec a_{2} \prec b_{2}$ holds in $L$, by Invariant A. It follows that any rainbow of visible inter-floor edges of $(G, f, g)$ is composed of edges between two floors $i$ and $i+1$. By Lemma 17, the end-vertices of such edges belong to $L_{i, 1}^{\prime}$ and to $L_{i+1,1}^{\prime}$.

Suppose, for a contradiction, that there exists a rainbow composed of six edges $\left(a_{1}, a_{2}\right)$, $\left(b_{1}, b_{2}\right),\left(c_{1}, c_{2}\right),\left(d_{1}, d_{2}\right),\left(e_{1}, e_{2}\right)$, and $\left(f_{1}, f_{2}\right)$ such that vertices $a_{1}, b_{1}, c_{1}, d_{1}, e_{1}$, and $f_{1}$ belong to $L_{i, 1}^{\prime}$, and such that vertices $a_{2}, b_{2}, c_{2}, d_{2}, e_{2}$, and $f_{2}$ belong to $L_{i+1,1}^{\prime}$. Suppose, w.l.o.g., that $a_{1} \prec b_{1} \prec c_{1} \prec d_{1} \prec e_{1} \prec f_{1} \prec f_{2} \prec e_{2} \prec d_{2} \prec c_{2} \prec b_{2} \prec a_{2}$. By Lemma 13 Algorithm Vertex-Ordering inserts $a_{1}, b_{1}, c_{1}, d_{1}, e_{1}$, and $f_{1}$ (resp. $f_{2}, e_{2}, d_{2}, c_{2}, b_{2}$, and $a_{2}$ ) into $L_{i, 1}^{\prime}$ (resp. into $L_{i+1,1}^{\prime}$ ) in this order. Suppose that $c_{1}$ is inserted into $L_{i, 1}^{\prime}$ before $d_{2}$ is inserted into $L_{i+1,1}^{\prime}$, the case in which $d_{2}$ is inserted into $L_{i+1,1}^{\prime}$ before $c_{1}$ is inserted into $L_{i, 1}^{\prime}$ being symmetric. Let $\left(G_{j}, f_{j}, g_{j}\right)$ be the graph that is processed by Algorithm Vertex-Ordering when $c_{1}$ is inserted into $L_{i, 1}^{\prime}$. By Lemma 23 , after $\left(G_{j}, f_{j}, g_{j}\right)$ has been processed by Algorithm Vertex-Ordering vertices $a_{2}$ and $b_{2}$ are not yet inserted into $L_{i+1,1}^{\prime}$. Further, by Lemma 24 , after $\left(G_{j}, f_{j}, g_{j}\right)$ has been processed either $a_{1}$ or $b_{1}$ does not belong to the border of $\left(G_{j}^{*}, f_{j}^{*}, g_{j}^{*}\right)$. Hence, either edge $\left(a_{1}, a_{2}\right)$ or edge $\left(b_{1}, b_{2}\right)$, respectively, does not exist. Such a contradiction proves the lemma.

(Proof of Statement (2)). If $f\left(a_{1}\right)=p_{1}, f\left(a_{2}\right)=p_{1}+1, f\left(b_{1}\right)=p_{2}>p_{1}$, and $f\left(b_{2}\right)=$ $p_{2}+1$, then inter-floor edges $\left(a_{1}, a_{2}\right)$ and $\left(b_{1}, b_{2}\right)$ do not nest, as either $a_{1} \prec a_{2} \prec b_{1} \prec b_{2}$ or $a_{1} \prec b_{1} \prec a_{2} \prec b_{2}$ holds in $L$, by Invariant A. It follows that any rainbow of semi-visible inter-floor edges of $(G, f, g)$ is composed of edges between two floors $i$ and $i+1$. By Lemma 18, the end-vertices of such edges belong to $L_{i, 1}^{\prime}$ and $L_{i+1,1}^{\prime \prime}$, or to $L_{i, 1}^{\prime \prime}$ and $L_{i+1,1}^{\prime}$. 
Suppose, for a contradiction, that there exists a rainbow composed of five edges $\left(a_{1}, a_{2}\right)$, $\left(b_{1}, b_{2}\right),\left(c_{1}, c_{2}\right),\left(d_{1}, d_{2}\right)$, and $\left(e_{1}, e_{2}\right)$ such that vertices $a_{1}, b_{1}, c_{1}, d_{1}$, and $e_{1}$ belong to $L_{i, 1}^{\prime}$, and such that vertices $a_{2}, b_{2}, c_{2}, d_{2}$, and $e_{2}$ belong to $L_{i+1,1}^{\prime \prime}$. Suppose, w.l.o.g., that $a_{1} \prec b_{1} \prec c_{1} \prec d_{1} \prec e_{1} \prec e_{2} \prec d_{2} \prec c_{2} \prec b_{2} \prec a_{2}$. By Lemma 13, $a_{1}, b_{1}, c_{1}, d_{1}$, and $e_{1}$ are inserted into $L_{i, 1}^{\prime}$ in this order. Let $\left(G_{j}, f_{j}, g_{j}\right)$ be the graph which is processed by Algorithm Vertex-Ordering when $c_{1}$ is inserted into $L_{i, 1}^{\prime}$. If $c_{2}$ has not yet been inserted into $L_{i+1,1}^{\prime \prime}$ after $\left(G_{j}, f_{j}, g_{j}\right)$ has been processed by Algorithm Vertex-Ordering, then by Lemma 24 after $\left(G_{j}, f_{j}, g_{j}\right)$ has been processed either $a_{1}$ or $b_{1}$ does not belong to the border of $\left(G_{j}^{*}, f_{j}^{*}, g_{j}^{*}\right)$. Hence, either edge $\left(a_{1}, a_{2}\right)$ or edge $\left(b_{1}, b_{2}\right)$, respectively, does not exist. If after $\left(G_{j}, f_{j}, g_{j}\right)$ has been processed by Algorithm Vertex-Ordering $c_{2}$ has been inserted into $L_{i+1,1}^{\prime \prime}$, then vertex $e_{2}$ has been inserted into $L_{i+1,1}^{\prime \prime}$, as well. Further, by Lemma 23 after $\left(G_{j}, f_{j}, g_{j}\right)$ has been processed by Algorithm Vertex-Ordering $e_{1}$ has not yet been inserted into $L_{i, 1}^{\prime}$. Hence, by Lemma 27, edge $\left(e_{1}, e_{2}\right)$ does not exist, thus providing a contradiction.

It can be analogously proved that there exists no rainbow composed of five edges $\left(a_{1}, a_{2}\right),\left(b_{1}, b_{2}\right),\left(c_{1}, c_{2}\right),\left(d_{1}, d_{2}\right)$, and $\left(e_{1}, e_{2}\right)$ such that vertices $a_{1}, b_{1}, c_{1}, d_{1}$, and $e_{1}$ belong to $L_{i, 1}^{\prime \prime}$, and such that vertices $a_{2}, b_{2}, c_{2}, d_{2}$, and $e_{2}$ belong to $L_{i+1,1}^{\prime}$.

Finally, suppose, for a contradiction, that there exists a rainbow composed of nine semi-visible inter-floor edges whose end-vertices belong to the $i$-th floor of $(G, f, g)$ and to the $(i+1)$-th floor of $(G, f, g)$. Then, by Lemma 18, either there exists a rainbow composed of four edges $\left(a_{1}, a_{2}\right),\left(b_{1}, b_{2}\right),\left(c_{1}, c_{2}\right),\left(d_{1}, d_{2}\right)$, and $\left(e_{1}, e_{2}\right)$ such that vertices $a_{1}, b_{1}, c_{1}, d_{1}$, and $e_{1}$ belong to $L_{i, 1}^{\prime}$, and such that vertices $a_{2}, b_{2}, c_{2}, d_{2}$, and $e_{2}$ belong to $L_{i+1,1}^{\prime \prime}$, or there exists a rainbow composed of five edges $\left(a_{1}, a_{2}\right),\left(b_{1}, b_{2}\right),\left(c_{1}, c_{2}\right),\left(d_{1}, d_{2}\right)$, and $\left(e_{1}, e_{2}\right)$ such that vertices $a_{1}, b_{1}, c_{1}, d_{1}$, and $e_{1}$ belong to $L_{i, 1}^{\prime \prime}$, and such that vertices $a_{2}, b_{2}, c_{2}, d_{2}$, and $e_{2}$ belong to $L_{i+1,1}^{\prime}$. Such a contradiction proves the lemma.

(Proof of Statement (3)). If $f\left(a_{1}\right)=p_{1}, f\left(a_{2}\right)=p_{1}+1, f\left(b_{1}\right)=p_{2}>p_{1}$, and $f\left(b_{2}\right)=$ $p_{2}+1$, then inter-floor edges $\left(a_{1}, a_{2}\right)$ and $\left(b_{1}, b_{2}\right)$ do not nest, as either $a_{1} \prec a_{2} \prec b_{1} \prec b_{2}$ or $a_{1} \prec b_{1} \prec a_{2} \prec b_{2}$ holds in $L$, by Invariant A. It follows that any rainbow of invisible inter-floor edges of $(G, f, g)$ is composed of edges between two floors $i$ and $i+1$. By Lemma 17, the end-vertices of such edges belong to $L_{i, 1}^{\prime \prime}$ and to $L_{i+1,1}^{\prime \prime}$.

By Lemma 16, every inter-floor edge of $(G, f, g)$ is an inter-floor edge of a graph $\left(G_{j}, f_{j}, g_{j}\right)$. Hence, if invisible edges $\left(a_{1}, a_{2}\right)$ and $\left(b_{1}, b_{2}\right)$ belong to graphs $\left(G_{j_{1}}, f_{j_{1}}, g_{j_{1}}\right)$ and $\left(G_{j_{2}}, f_{j_{2}}, g_{j_{2}}\right)$, respectively, and $j_{1}<j_{2}$, then $a_{1} \prec b_{1} \prec a_{2} \prec b_{2}$, hence $\left(a_{1}, a_{2}\right)$ and $\left(b_{1}, b_{2}\right)$ do not nest. It follows that the size of every rainbow of invisible inter-floor edges of $(G, f, g)$ is not larger than the maximum size of a rainbow of inter-floor edges in a graph $\left(G_{j}, f_{j}, g_{j}\right)$, for some $j$, when the vertices of $\left(G_{j}, f_{j}, g_{j}\right)$ are ordered as in $L$. It follows from Invariant $\mathrm{A}$ that the inter-floor edges of $(G, f, g)$ belonging to a graph $\left(G_{j}, f_{j}, g_{j}\right)$ have their end-vertices whose order in $L$ is the same order as the one computed by Algorithm Vertex-Ordering when applied to $\left(G_{j}, f_{j}, g_{j}\right)$, and the lemma follows.

Lemma $38 q_{\text {inter }}(n)=O(\log n)$.

Proof: Every inter-floor edge is either visible, or semi-visible, or invisible, by Lemma 14. By definition, the size of a rainbow of inter-floor edges among graphs $\left(G_{1}, f_{1}, g_{1}\right), \ldots$, $\left(G_{l+1}, f_{l+1}, g_{l+1}\right)$ is at most $q_{\text {inter }}(n / 2)$. By Lemmata 37 and 35 , all the visible, semi-visible, and invisible inter-floor edges of $G$ can be embedded into at most 5,8 , and $q_{\text {inter }}(n / 2)$ queues, respectively. Hence, $q_{\text {inter }}(n)=13+q_{\text {inter }}(n / 2)=O(\log n)$. 
Lemma 39 (1) The size of a rainbow of visible intra-floor edges of $(G, f, g)$ is at most seven. (2) The size of a rainbow of semi-visible intra-floor edges of $(G, f, g)$ is at most twelve. (3) The size of a rainbow of invisible intra-floor edges of $(G, f, g)$ is at most the maximum size of a rainbow of inter-floor edges among graphs $\left(G_{1}, f_{1}, g_{1}\right), \ldots$,

$\left(G_{l+1}, f_{l+1}, g_{l+1}\right)$ plus the maximum size of a rainbow of intra-floor edges among graphs $\left(G_{1}, f_{1}, g_{1}\right), \ldots,\left(G_{l+1}, f_{l+1}, g_{l+1}\right)$.

Proof: (Proof of Statement (1)). By Lemma 20, every visible intra-floor edge either connects two vertices in the same list $L_{p, q}^{\prime}$, for some $p$ and $q$, or it connects two vertices in lists $L_{p, q}^{\prime}$ and $L_{p, q+1}^{\prime}$, for some $p$ and $q$.

We first prove that the size of a rainbow of visible intra-floor edges whose end-vertices are in the same list $L_{p, q}^{\prime}$, for any $p$ and $q$, is at most two.

If $f\left(a_{1}\right)=p_{1}, f\left(a_{2}\right)=p_{1}, f\left(b_{1}\right)=p_{2}>p_{1}$, and $f\left(b_{2}\right)=p_{2}$, then intra-floor edges $\left(a_{1}, a_{2}\right)$ and $\left(b_{1}, b_{2}\right)$ do not nest, as $a_{1}, a_{2} \prec b_{1}, b_{2}$ holds in $L$, by Invariant A. Further, if $a_{1}$ and $a_{2}$ belong to a list $L_{p, q}^{\prime}$ and $b_{1}$ and $b_{2}$ belong to a list $L_{p, r}^{\prime}$, for some $p, q$, and $r$ with $r>q$, then $\left(a_{1}, a_{2}\right)$ and $\left(b_{1}, b_{2}\right)$ do not nest, as $a_{1}, a_{2} \prec b_{1}, b_{2}$ holds in $L$, by construction of $L$. It follows that any rainbow of visible intra-floor edges of $(G, f, g)$ whose both endvertices are in the same list $L_{p, q}^{\prime}$, for any $p$ and $q$, is composed of edges whose end-vertices are in the same list $L_{p, q}^{\prime}$, for some fixed $p$ and $q$.

Suppose, for a contradiction, that there exists a rainbow composed of three edges $\left(a_{1}, a_{2}\right),\left(b_{1}, b_{2}\right)$, and $\left(c_{1}, c_{2}\right)$ such that vertices $a_{1}, b_{1}, c_{1}, a_{2}, b_{2}$, and $c_{2}$ belong to $L_{p, q}^{\prime}$, for some fixed $p$ and $q$. Suppose, w.l.o.g., that $a_{1} \prec b_{1} \prec c_{1} \prec c_{2} \prec b_{2} \prec a_{2}$. By Lemma 13, $a_{1}, b_{1}, c_{1}, c_{2}, b_{2}$, and $a_{2}$ are inserted into $L_{p, q}^{\prime}\left(\right.$ resp. into $\left.L_{p, q}^{\prime}\right)$ in this order. Let $\left(G_{j}, f_{j}, g_{j}\right)$ be the graph that is processed by Algorithm Vertex-Ordering when $c_{1}$ is inserted into $L_{p, q}^{\prime}$. By Lemma 23, after $\left(G_{j}, f_{j}, g_{j}\right)$ has been processed by Algorithm Vertex-Ordering vertices $a_{2}$ and $b_{2}$ are not yet inserted into $L_{p, q}^{\prime}$. Further, by Lemma 24 , after $\left(G_{j}, f_{j}, g_{j}\right)$ has been processed either $a_{1}$ or $b_{1}$ does not belong to the border of $\left(G_{j}^{*}, f_{j}^{*}, g_{j}^{*}\right)$. Hence, either edge $\left(a_{1}, a_{2}\right)$ or edge $\left(b_{1}, b_{2}\right)$, respectively, does not exist, thus providing a contradiction.

We now prove that the size of a rainbow of visible intra-floor edges whose end-vertices are in two lists $L_{p, q}^{\prime}$ and $L_{p, q+1}^{\prime}$, for any $p$ and $q$, is at most five.

If $f\left(a_{1}\right)=p_{1}, f\left(a_{2}\right)=p_{1}, f\left(b_{1}\right)=p_{2}>p_{1}$, and $f\left(b_{2}\right)=p_{2}$, then intra-floor edges $\left(a_{1}, a_{2}\right)$ and $\left(b_{1}, b_{2}\right)$ do not nest, as $a_{1}, a_{2} \prec b_{1}, b_{2}$ holds in $L$, by Invariant A. Further, if $a_{1}$ belongs to a list $L_{p, q}^{\prime}, a_{2}$ belongs to a list $L_{p, q+1}^{\prime}, b_{1}$ belongs to a list $L_{p, r}^{\prime}$, and $b_{2}$ belongs to a list $L_{p, r+1}^{\prime}$, for some $p, q$, and $r>q$, then $\left(a_{1}, a_{2}\right)$ and $\left(b_{1}, b_{2}\right)$ do not nest, as $a_{1} \prec a_{2} \prec b_{1} \prec b_{2}$ or $a_{1} \prec b_{1} \prec a_{2} \prec b_{2}$ holds in $L$, by construction of $L$. It follows that any rainbow of visible intra-floor edges of $(G, f, g)$ whose end-vertices are in two lists $L_{p, q}^{\prime}$ and $L_{p, q+1}^{\prime}$, for any $p$ and $q$, is composed of edges whose end-vertices are in the two lists $L_{p, q}^{\prime}$ and $L_{p, q+1}^{\prime}$, for some fixed $p$ and $q$.

Suppose, for a contradiction, that there exists a rainbow composed of six edges $\left(a_{1}, a_{2}\right)$, $\left(b_{1}, b_{2}\right),\left(c_{1}, c_{2}\right),\left(d_{1}, d_{2}\right),\left(e_{1}, e_{2}\right)$, and $\left(f_{1}, f_{2}\right)$ such that vertices $a_{1}, b_{1}, c_{1}, d_{1}, e_{1}$, and $f_{1}$ belong to $L_{p, q}^{\prime}$, and such that vertices $a_{2}, b_{2}, c_{2}, d_{2}, e_{2}$, and $f_{2}$ belong to $L_{p, q+1}^{\prime}$, for some fixed $p$ and $q$. Suppose, w.l.o.g., that $a_{1} \prec b_{1} \prec c_{1} \prec d_{1} \prec e_{1} \prec f_{1} \prec f_{2} \prec e_{2} \prec d_{2} \prec$ $c_{2} \prec b_{2} \prec a_{2}$. By Lemma 13, $a_{1}, b_{1}, c_{1}, d_{1}, e_{1}$, and $f_{1}$ (resp. $f_{2}, e_{2}, d_{2}, c_{2}, b_{2}$, and $a_{2}$ ) are inserted into $L_{p, q}^{\prime}$ (resp. into $L_{p, q+1}^{\prime}$ ) in this order. Suppose that $c_{1}$ is inserted into $L_{p, q}^{\prime}$ before $d_{2}$ is inserted into $L_{p, q+1}^{\prime}$, the case in which $d_{2}$ is inserted into $L_{p, q+1}^{\prime}$ before $c_{1}$ is inserted into $L_{p, q}^{\prime}$ being symmetric. Let $\left(G_{j}, f_{j}, g_{j}\right)$ be the graph that is processed by Algorithm Vertex-Ordering when $c_{1}$ is inserted into $L_{p, q}^{\prime}$. By Lemma 23 , after $\left(G_{j}, f_{j}, g_{j}\right)$ 
has been processed by Algorithm Vertex-Ordering vertices $a_{2}$ and $b_{2}$ are not yet inserted into $L_{i+1,1}^{\prime}$. Further, by Lemma 24 , after $\left(G_{j}, f_{j}, g_{j}\right)$ has been processed either $a_{1}$ or $b_{1}$ does not belong to the border of $\left(G_{j}^{*}, f_{j}^{*}, g_{j}^{*}\right)$. Hence, either edge $\left(a_{1}, a_{2}\right)$ or edge $\left(b_{1}, b_{2}\right)$, respectively, does not exist, thus providing a contradiction.

Finally, suppose, for a contradiction, that there exists a rainbow composed of eight visible intra-floor edges of $(G, f, g)$. Then, by Lemma 20 either there exists a rainbow composed of three edges $\left(a_{1}, a_{2}\right),\left(b_{1}, b_{2}\right)$, and $\left(c_{1}, c_{2}\right)$ such that vertices $a_{1}, b_{1}, c_{1}, a_{2}, b_{2}$, and $c_{2}$ belong to $L_{p, q}^{\prime}$, for some fixed $p$ and $q$, or there exists a rainbow composed of six edges $\left(a_{1}, a_{2}\right),\left(b_{1}, b_{2}\right),\left(c_{1}, c_{2}\right),\left(d_{1}, d_{2}\right),\left(e_{1}, e_{2}\right)$, and $\left(f_{1}, f_{2}\right)$ such that vertices $a_{1}, b_{1}, c_{1}$, $d_{1}, e_{1}$, and $f_{1}$ belong to $L_{p, q}^{\prime}$, and such that vertices $a_{2}, b_{2}, c_{2}, d_{2}, e_{2}$, and $f_{2}$ belong to $L_{p, q+1}^{\prime}$, for some fixed $p$ and $q$. Such a contradiction proves the lemma.

(Proof of Statement (2)). By Lemma 21, every semi-visible intra-floor edge either connects two vertices in lists $L_{p, q}^{\prime}$ and $L_{p, q}^{\prime \prime}$, for some $p$ and $q$, or it connects two vertices in lists $L_{p, q}^{\prime}$ and $L_{p, q+1}^{\prime \prime}$, for some $p$ and $q$, or it connects two vertices in lists $L_{p, q}^{\prime \prime}$ and $L_{p, q+1}^{\prime}$, for some $p$ and $q$.

We first prove that the size of a rainbow of semi-visible intra-floor edges whose endvertices are in lists $L_{p, q}^{\prime}$ and $L_{p, q}^{\prime \prime}$, for any $p$ and $q$, is at most four.

If $f\left(a_{1}\right)=p_{1}, f\left(a_{2}\right)=p_{1}, f\left(b_{1}\right)=p_{2}>p_{1}$, and $f\left(b_{2}\right)=p_{2}$, then intra-floor edges $\left(a_{1}, a_{2}\right)$ and $\left(b_{1}, b_{2}\right)$ do not nest, as $a_{1} \prec a_{2} \prec b_{1} \prec b_{2}$ holds in $L$, by Invariant A. Further, if $a_{1}$ belongs to a list $L_{p, q}^{\prime}, a_{2}$ belongs to a list $L_{p, q}^{\prime \prime}, b_{1}$ belongs to a list $L_{p, r}^{\prime}$, and $b_{2}$ belongs to a list $L_{p, r}^{\prime \prime}$, for some $p, q$, and $r>q$, then $\left(a_{1}, a_{2}\right)$ and $\left(b_{1}, b_{2}\right)$ do not nest, as $a_{1} \prec a_{2} \prec b_{1} \prec b_{2}$ holds in $L$, by construction of $L$. It follows that any rainbow of semi-visible intra-floor edges of $(G, f, g)$ whose end-vertices are in lists $L_{p, q}^{\prime}$ and $L_{p, q}^{\prime \prime}$, for any $p$ and $q$, is composed of edges whose end-vertices are in lists $L_{p, q}^{\prime}$ and $L_{p, q}^{\prime \prime}$, for some fixed $p$ and $q$.

Suppose, for a contradiction, that there exists a rainbow composed of five edges $\left(a_{1}, a_{2}\right)$, $\left(b_{1}, b_{2}\right),\left(c_{1}, c_{2}\right),\left(d_{1}, d_{2}\right)$, and $\left(e_{1}, e_{2}\right)$ such that vertices $a_{1}, b_{1}, c_{1}, d_{1}$, and $e_{1}$ belong to $L_{p, q}^{\prime}$, and such that vertices $a_{2}, b_{2}, c_{2}, d_{2}$, and $e_{2}$ belong to $L_{p, q}^{\prime \prime}$. Suppose, w.l.o.g., that $a_{1} \prec b_{1} \prec c_{1} \prec d_{1} \prec e_{1}$. By Lemma 13, $a_{1}, b_{1}, c_{1}, d_{1}$, and $e_{1}$ are inserted into $L_{p, q}^{\prime}$ in this order. Let $\left(G_{j}, f_{j}, g_{j}\right)$ be the graph that is processed by Algorithm Vertex-Ordering when $c_{1}$ is inserted into $L_{p, q}^{\prime}$. If $c_{2}$ has not yet been inserted into $L_{p, q}^{\prime \prime}$ by Algorithm Vertex-Ordering after processing $\left(G_{j}, f_{j}, g_{j}\right)$, then $a_{2}$ and $b_{2}$ have not yet been inserted into $L_{p, q}^{\prime \prime}$ by Algorithm Vertex-Ordering after processing $\left(G_{j}, f_{j}, g_{j}\right)$, as well. Further, by Lemma 24 , after $\left(G_{j}, f_{j}, g_{j}\right)$ has been processed either $a_{1}$ or $b_{1}$ does not belong to the border of $\left(G_{j}^{*}, f_{j}^{*}, g_{j}^{*}\right)$. Hence, either edge $\left(a_{1}, a_{2}\right)$ or edge $\left(b_{1}, b_{2}\right)$, respectively, does not exist, by Lemma 32. If $c_{2}$ has already been inserted into $L_{p, q}^{\prime \prime}$ by Algorithm VertexOrdering after processing $\left(G_{j}, f_{j}, g_{j}\right)$, then $d_{2}$ and $e_{2}$ have been inserted into $L_{p, q}^{\prime \prime}$ by Algorithm Vertex-Ordering after processing $\left(G_{j}, f_{j}, g_{j}\right)$, as well. However, $e_{1}$ has not been inserted by Algorithm Vertex-Ordering after processing $\left(G_{j}, f_{j}, g_{j}\right)$, by Lemma 23 . Hence, by Lemma 32 , edge $\left(e_{1}, e_{2}\right)$ does not exist, thus providing a contradiction.

It can be analogously proved that the size of a rainbow of semi-visible intra-floor edges whose end-vertices are in lists $L_{p, q}^{\prime}$ and $L_{p, q+1}^{\prime \prime}$, for any $p$ and $q$, is at most four, and that the size of a rainbow of semi-visible intra-floor edges whose end-vertices are in lists $L_{p, q}^{\prime}$ and $L_{p, q-1}^{\prime \prime}$, for any $p$ and $q$, is at most four.

Finally, suppose, for a contradiction, that there exists a rainbow composed of thirteen semi-visible inter-floor edges whose end-vertices belong to the $p$-th floor of $(G, f, g)$. Then, by Lemma 21, either there exists a rainbow composed of five edges $\left(a_{1}, a_{2}\right),\left(b_{1}, b_{2}\right),\left(c_{1}, c_{2}\right)$, 
$\left(d_{1}, d_{2}\right)$, and $\left(e_{1}, e_{2}\right)$ such that vertices $a_{1}, b_{1}, c_{1}, d_{1}$, and $e_{1}$ belong to $L_{p, q}^{\prime}$, and such that vertices $a_{2}, b_{2}, c_{2}, d_{2}$, and $e_{2}$ belong to $L_{p, q}^{\prime \prime}$, or there exists a rainbow composed of five edges $\left(a_{1}, a_{2}\right),\left(b_{1}, b_{2}\right),\left(c_{1}, c_{2}\right),\left(d_{1}, d_{2}\right)$, and $\left(e_{1}, e_{2}\right)$ such that vertices $a_{1}, b_{1}, c_{1}, d_{1}$, and $e_{1}$ belong to $L_{p, q}^{\prime}$, and such that vertices $a_{2}, b_{2}, c_{2}, d_{2}$, and $e_{2}$ belong to $L_{p, q+1}^{\prime \prime}$, or there exists a rainbow composed of five edges $\left(a_{1}, a_{2}\right),\left(b_{1}, b_{2}\right),\left(c_{1}, c_{2}\right),\left(d_{1}, d_{2}\right)$, and $\left(e_{1}, e_{2}\right)$ such that vertices $a_{1}, b_{1}, c_{1}, d_{1}$, and $e_{1}$ belong to $L_{p, q}^{\prime \prime}$, and such that vertices $a_{2}, b_{2}, c_{2}, d_{2}$, and $e_{2}$ belong to $L_{p, q-1}^{\prime \prime}$. Such a contradiction proves the lemma.

(Proof of Statement (3)). By Lemma 22, every invisible intra-floor edge either connects two vertices in list $L_{p, q}^{\prime \prime}$, for some $p$ and $q$, or it connects two vertices in lists $L_{p, q}^{\prime \prime}$ and $L_{p, q+1}^{\prime \prime}$, for some $p$ and $q$.

We first prove that the size of a rainbow of invisible intra-floor edges whose end-vertices are in list $L_{p, q}^{\prime \prime}$, for any $p$ and $q$, is at most the maximum size of a rainbow of intra-floor edges among graphs $\left(G_{1}, f_{1}, g_{1}\right), \ldots,\left(G_{l+1}, f_{l+1}, g_{l+1}\right)$.

If $f\left(a_{1}\right)=p_{1}, f\left(a_{2}\right)=p_{1}, f\left(b_{1}\right)=p_{2}>p_{1}$, and $f\left(b_{2}\right)=p_{2}$, then intra-floor edges $\left(a_{1}, a_{2}\right)$ and $\left(b_{1}, b_{2}\right)$ do not nest, as $a_{1} \prec a_{2} \prec b_{1} \prec b_{2}$ holds in $L$, by Invariant A. Further, if $a_{1}$ belongs to a list $L_{p, q}^{\prime \prime}, a_{2}$ belongs to a list $L_{p, q}^{\prime \prime}, b_{1}$ belongs to a list $L_{p, r}^{\prime \prime}$, and $b_{2}$ belongs to a list $L_{p, r}^{\prime \prime}$, for some $p, q$, and $r>q$, then $\left(a_{1}, a_{2}\right)$ and $\left(b_{1}, b_{2}\right)$ do not nest, as $a_{1} \prec a_{2} \prec b_{1} \prec b_{2}$ holds in $L$, by construction of $L$. Moreover, if $a_{1}, a_{2}, b_{1}$, and $b_{2}$ belong to a list $L_{p, q}^{\prime \prime}$ and if $\left(a_{1}, a_{2}\right)$ belongs to a graph $\left(G_{j_{1}}, f_{j_{1}}, g_{j_{1}}\right)$ and $\left(b_{1}, b_{2}\right)$ belongs to a graph $\left(G_{j_{2}}, f_{j_{2}}, g_{j_{2}}\right)$, with $j_{1}<j_{2}$, then $\left(a_{1}, a_{2}\right)$ and $\left(b_{1}, b_{2}\right)$ do not nest, as $a_{1} \prec a_{2} \prec b_{1} \prec b_{2}$ holds in $L$, by the algorithm's construction. It follows that any rainbow of invisible interfloor edges of $(G, f, g)$ whose end-vertices are in the same list $L_{p, q}^{\prime \prime}$, for any $p$ and $q$, is composed of edges whose end-vertices are in the same list $L_{p, q}^{\prime \prime}$, for some fixed $p$ and $q$, and belong to some graph $\left(G_{j}, f_{j}, g_{j}\right)$, for some fixed $j$. Since the order of the vertices of the $r$-th floor of $\left(G_{j}, f_{j}, g_{j}\right)$ in $L$ is the same order of the one computed by Algorithm Vertex-Ordering when applied to $\left(G_{j}, f_{j}, g_{j}\right)$, the statement follows.

We now prove that the size of a rainbow of invisible intra-floor edges whose end-vertices are in lists $L_{p, q}^{\prime \prime}$ and $L_{p, q+1}^{\prime \prime}$, for any $p$ and $q$, is at most the maximum size of a rainbow of inter-floor edges among graphs $\left(G_{1}, f_{1}, g_{1}\right), \ldots,\left(G_{l+1}, f_{l+1}, g_{l+1}\right)$.

If $f\left(a_{1}\right)=p_{1}, f\left(a_{2}\right)=p_{1}, f\left(b_{1}\right)=p_{2}>p_{1}$, and $f\left(b_{2}\right)=p_{2}$, then intra-floor edges $\left(a_{1}, a_{2}\right)$ and $\left(b_{1}, b_{2}\right)$ do not nest, as $a_{1} \prec a_{2} \prec b_{1} \prec b_{2}$ holds in $L$, by Invariant A. Further, if $a_{1}$ belongs to a list $L_{p, q}^{\prime \prime}, a_{2}$ belongs to a list $L_{p, q+1}^{\prime \prime}, b_{1}$ belongs to a list $L_{p, r}^{\prime \prime}$, and $b_{2}$ belongs to a list $L_{p, r+1}^{\prime \prime}$, for some $p, q$, and $r>q$, then $\left(a_{1}, a_{2}\right)$ and $\left(b_{1}, b_{2}\right)$ do not nest, as either $a_{1} \prec a_{2} \prec b_{1} \prec b_{2}$ or $a_{1} \prec b_{1} \prec a_{2} \prec b_{2}$ holds in $L$, by construction of $L$. Moreover, if $a_{1}$ and $b_{1}$ belong to a list $L_{p, q}^{\prime \prime}$, if $a_{2}$ and $b_{2}$ belong to a list $L_{p, q+1}^{\prime \prime}$, if $\left(a_{1}, a_{2}\right)$ belongs to a graph $\left(G_{j_{1}}, f_{j_{1}}, g_{j_{1}}\right)$, and if $\left(b_{1}, b_{2}\right)$ belongs to a graph $\left(G_{j_{2}}, f_{j_{2}}, g_{j_{2}}\right)$, with $j_{1}<j_{2}$, then $\left(a_{1}, a_{2}\right)$ and $\left(b_{1}, b_{2}\right)$ do not nest, as $a_{1} \prec b_{1} \prec a_{2} \prec b_{2}$ holds in $L$, by the algorithm's construction. It follows that any rainbow of invisible intra-floor edges of $(G, f, g)$ whose end-vertices are in lists $L_{p, q}^{\prime \prime}$ and $L_{p, q+1}^{\prime \prime}$, for any $p$ and $q$, is composed of edges whose end-vertices are in lists $L_{p, q}^{\prime \prime}$ and $L_{p, q+1}^{\prime \prime}$, for some fixed $p$ and $q$, and belong to some graph $\left(G_{j}, f_{j}, g_{j}\right)$, for some fixed $j$. The order of the vertices of the $r$-th floor of $\left(G_{j}, f_{j}, g_{j}\right)$ in $L$ is the same order of the one computed by Algorithm Vertex-Ordering when applied to $\left(G_{j}, f_{j}, g_{j}\right)$. However, while all the vertices in $L_{p, q}^{\prime \prime}$ precede each vertex in $L_{p, q+1}^{\prime \prime}$ in $L$, all the vertices in $L_{p, q}^{\prime \prime}$ follow each vertex in $L_{p, q+1}^{\prime \prime}$ in the vertex ordering of $\left(G_{j}, f_{j}, g_{j}\right)$ computed by Algorithm Vertex-Ordering. Nevertheless, by Lemma 36, the maximum size of a rainbow of edges between vertices in $L_{p, q}^{\prime \prime}$ and vertices in $L_{p, q+1}^{\prime \prime}$ in $L$ is equal to the maximum size of a rainbow of edges between vertices in $L_{p, q}^{\prime \prime}$ and vertices in $L_{p, q+1}^{\prime \prime}$ when 
such vertices are ordered as computed by Algorithm Vertex-Ordering, and the statement follows.

Since every invisible intra-floor edges of $(G, f, g)$ is either an edge between two vertices of $L_{p, q}^{\prime \prime}$ or an edge between a vertex of $L_{p, q}^{\prime \prime}$ and a vertex of $L_{p, q+1}^{\prime \prime}$, by Lemma 22 , it follows that the maximum size of a rainbow of invisible intra-floor edges of $(G, f, g)$ is at most the maximum size of a rainbow of inter-floor edges among graphs $\left(G_{1}, f_{1}, g_{1}\right), \ldots$, $\left(G_{l+1}, f_{l+1}, g_{l+1}\right)$ plus the maximum size of a rainbow of intra-floor edges among graphs $\left(G_{1}, f_{1}, g_{1}\right), \ldots,\left(G_{l+1}, f_{l+1}, g_{l+1}\right)$, thus proving the lemma.

Lemma $40 q_{\text {intra }}(n)=O\left(\log ^{2} n\right)$.

Proof: Every intra-floor edge is either visible, or semi-visible, or invisible, by Lemma 14 . By definition, the size of a rainbow of inter-floor edges among graphs $\left(G_{1}, f_{1}, g_{1}\right), \ldots$, $\left(G_{l+1}, f_{l+1}, g_{l+1}\right)$ is at most $q_{\text {inter }}(n / 2)$ and the size of a rainbow of intra-floor edges among graphs $\left(G_{1}, f_{1}, g_{1}\right), \ldots,\left(G_{l+1}, f_{l+1}, g_{l+1}\right)$ is at most $q_{\text {inter }}(n / 2)$. By Lemmata 39 and 35, all the visible, semi-visible, and invisible inter-floor edges of $G$ can be embedded into at most 7,12 , and $q_{\text {inter }}(n / 2)+q_{\text {intra }}(n / 2)$ queues, respectively. By Lemma 38 , $q_{\text {inter }}(n / 2)=O(\log n)$. Hence, $q_{\text {intra }}(n)=O(\log n)+q_{\text {intra }}(n / 2)=O\left(\log ^{2} n\right)$.

Theorem 1 Every n-vertex level-2-connected graph $G$ has $O\left(\log ^{2} n\right)$ queue number.

Proof: Consider any level-2-connected graph $G$ with $n$ vertices. Let $f(v)=1$, for every vertex $v$ in $G$. Let $g$ be any edge on the outer face of $G$. By Lemma $4(G, f, g)$ is a floored graph. Further, $G$ has $n-2$ non-border vertices. By Lemma 15, every edge of $(G, f, g)$ is either an inter-floor edge or an intra-floor edge. Hence, $q(G, f, g) \leq$ $q_{\text {intra }}(G, f, g)+q_{\text {inter }}(G, f, g)$. By definition, $q_{\text {intra }}(G, f, g) \leq q_{\text {intra }}(n)$ and $q_{\text {inter }}(G, f, g) \leq$ $q_{\text {inter }}(n)$. Hence, $q(G, f, g) \leq q_{\text {intra }}(n)+q_{\text {inter }}(n)$. By Lemmata 38 and $40, q(G, f, g)=$ $O\left(\log ^{2} n\right)+O(\log n)=O\left(\log ^{2} n\right)$, and the theorem follows.

Lemma 41 (Dujmović and Wood [11]) Let D be a q-queue subdivision of a graph $G$ with at most one subdivision vertex per edge. Then $G$ has a $2 q(q+1)$-queue layout.

Theorem 2 Every n-vertex planar graph has $O\left(\log ^{4} n\right)$ queue number. A queue layout with such a queue number can be computed in polynomial time.

Proof: By Lemma 1, for every planar graph $G$, an $O(n)$-vertex level-2-connected graph $G^{*}$ exists such that $G^{*}$ contains a 1 -subdivision $G^{\prime}$ of $G$ as a subgraph. By Theorem 1, $G^{*}$ has $O\left(\log ^{2} n\right)$ queue number, hence $G^{\prime}$ has $O\left(\log ^{2} n\right)$ queue number. By Lemma 41, $G$ has $O\left(\log ^{4} n\right)$ queue number. Finally, it is easy to see that the algorithm for constructing a vertex ordering of $G$ can be implemented in polynomial time.

The bound of Theorem 2, together with the following theorem, immediately implies an $O(n$ polylog $n)$ upper bound on the volume requirements of $3 \mathrm{D}$ straight-line crossing-free drawings of planar graphs.

Theorem 3 (Dujmović, Morin, and Wood [8]) Let $\mathcal{G}$ be a proper minor-closed family of graphs, and let $\mathcal{F}(n)$ be a family of functions closed under multiplication. The following are equivalent: (a) Every n-vertex graph in $\mathcal{G}$ has a $\mathcal{F}(n) \times \mathcal{F}(n) \times O(n)$ drawing, (b) $\mathcal{G}$ has $\mathcal{F}(n)$ track number $\operatorname{tn}(\mathcal{G}) \in \mathcal{F}(n)$, and $(c) \mathcal{G}$ has $\mathcal{F}(n)$ queue number qn $(\mathcal{G}) \in \mathcal{F}(n)$. 
Some results of Dujmović et al. [8] related to Theorem 3 lead us to precisely determine the value $k$ for the $O\left(n \log ^{k} n\right)$ volume upper bound for 3D straight-line crossing-free drawings of planar graphs.

Theorem 4 Every planar graph has a 3D straight-line crossing-free drawing in $O\left(n \log ^{32} n\right)$ volume.

Proof: Every graph $G$ with acyclic chromatic number $c$ and queue number $q$ has track number $\operatorname{tn}(G) \leq c(2 q)^{c-1}[8]$, where the acyclic chromatic number of a graph $G$ is the minimum number of colors such that $G$ admits a proper coloring in which each pair of colors induces a forest. Since every planar graph has acyclic chromatic number at most five [2] and queue number at most $O\left(\log ^{4} n\right)$ (by Theorem 2), then every planar graph has track number at most $\operatorname{tn}(G) \leq O\left(\log ^{4} n\right)^{4}=O\left(\log ^{16} n\right)$. Since every graph $G$ has an $O(\operatorname{tn}(G)) \times O(\operatorname{tn}(G)) \times O(n)$ volume 3D straight-line crossing-free drawing [8], the theorem follows.

\section{Conclusions}

In this paper we have shown that planar graphs have $O\left(\log ^{4} n\right)$ queue number, improving upon the previously best known $O(\sqrt{n})$ bound. Determining the asymptotic behavior of the queue number of planar graphs remains a challenging open problem for which, as far as we know, no super-constant lower bound is known. While we find unlikely that the techniques introduced in this paper can lead to determine a constant upper bound for the queue number of planar graphs, it is possible that, by directly handling cut-vertices in a planar graph decomposition similar to the one we presented, an $O\left(\log ^{2} n\right)$ upper bound can be achieved. We also leave to further research work to design a time-efficient implementation of our algorithm.

As a consequence of our results on the queue number of planar graphs and of a correspondence between queue layouts and 3D straight-line crossing-free drawings introduced by Dujmović et al. [8], planar graphs admit 3D straight-line crossing-free drawings in $O\left(n \log ^{c} n\right)$ volume, for some constant $c$. The question of whether such a volume bound can be reduced to linear remains one of the main unsolved problems in Graph Drawing. In particular, we find fundamental to understand whether small volume can be achieved while obtaining a good aspect ratio for the drawing.

\section{References}

[1] S. N. Bhatt, F. R. K. Chung, F. T. Leighton, and A. L. Rosenberg. Scheduling tree-dags using FIFO queues: A control-memory trade-off. Journal of Parallel and Distributed Computing, 33(1):55-68, 1996.

[2] O. V. Borodin. On acyclic colourings of planar graphs. Discrete Mathematics, 25:211$236,1979$.

[3] F. J. Brandenburg, D. Eppstein, M. T. Goodrich, S. G. Kobourov, G. Liotta, and P. Mutzel. Selected open problems in graph drawing. In G. Liotta, editor, Graph Drawing (GD '03), volume 2912 of LNCS, pages 515-539, 2004. 
[4] J. F. Buss and P. W. Shor. On the pagenumber of planar graphs. In Symposium on Theory of Computing (STOC '84), pages 98-100. ACM, 1984.

[5] R. F. Cohen, P. Eades, T. Lin, and F. Ruskey. Three-dimensional graph drawing. Algorithmica, 17(2):199-208, 1997.

[6] E. D. Demaine, J. S. B. Mitchell, and J. O'Rourke. The Open Problems Project. http://maven.smith.edu/ orourke/TOPP/Welcome.html.

[7] V. Dujmović. Track Layouts of Graphs. PhD thesis, School of Computer Science, McGill University, Montreal, Canada, 2003.

[8] V. Dujmović, P. Morin, and D. R. Wood. Layout of graphs with bounded tree-width. SIAM Journal on Computing, 34(3):553-579, 2005.

[9] V. Dujmović and D. R. Wood. On linear layouts of graphs. Discrete Mathematics 6 Theoretical Computer Science, 6(2):339-358, 2004.

[10] V. Dujmović and D. R. Wood. Three-dimensional grid drawings with sub-quadratic volume, pages 55-66. In J. Pach, editor, Towards a Theory of Geometric Graphs. Contemporary Mathematics, American Mathematical Society, 2004.

[11] V. Dujmović and D. R. Wood. Stacks, queues and tracks: Layouts of graph subdivisions. Discrete Mathematics \& Theoretical Computer Science, 7(1):155-202, 2005.

[12] T. Endo. The pagenumber of toroidal graphs is at most seven. Discrete Mathematics, 175(1-3):87-96, 1997.

[13] H. Enomoto, T. Nakamigawa, and K. Ota. On the pagenumber of complete bipartite graphs. Journal of Combinatorial Theory, Series B, 71(1):111-120, 1997.

[14] S. Felsner, G. Liotta, and S. K. Wismath. Straight-line drawings on restricted integer grids in two and three dimensions. Journal of Graph Algorithms 8 Applications, 7(4):363-398, 2003.

[15] R. A. Games. Optimal book embeddings of the FFT, Benes, and barrel shifter networks. Algorithmica, 1(2):233-250, 1986.

[16] J. L. Ganley and L. S. Heath. The pagenumber of k-trees is $\mathrm{O}(\mathrm{k})$. Discrete Applied Mathematics, 109(3):215-221, 2001.

[17] T. Hasunuma and Y. Shibata. Embedding De Bruijn, Kautz and shuffle-exchange networks in books. Discrete Applied Mathematics, 78(1-3):103-116, 1997.

[18] L. S. Heath. Embedding planar graphs in seven pages. In Foundations of Computer Science (FOCS '84), pages 74-83. IEEE, 1984.

[19] L. S. Heath and S. Istrail. The pagenumber of genus $g$ graphs is $O(g)$. Journal of the ACM, 39(3):479-501, 1992.

[20] L. S. Heath, F. T. Leighton, and A. L. Rosenberg. Comparing queues and stacks as mechanisms for laying out graphs. SIAM J. Discrete Math., 5(3):398-412, 1992. 
[21] L. S. Heath and S. V. Pemmaraju. Stack and queue layouts of posets. SIAM Journal on Discrete Mathematics, 10(4):599-625, 1997.

[22] L. S. Heath and S. V. Pemmaraju. Stack and queue layouts of directed acyclic graphs: Part II. SIAM Journal on Computing, 28(5):1588-1626, 1999.

[23] L. S. Heath, S. V. Pemmaraju, and A. N. Trenk. Stack and queue layouts of directed acyclic graphs: Part I. SIAM Journal on Computing, 28(4):1510-1539, 1999.

[24] L. S. Heath and A. L. Rosenberg. Laying out graphs using queues. SIAM Journal on Computing, 21(5):927-958, 1992.

[25] S. M. Malitz. Genus g graphs have pagenumber $O(\sqrt{g})$. Journal of Algorithms, 17(1):85-109, 1994.

[26] S. M. Malitz. Graphs with $e$ edges have pagenumber $O(\sqrt{e})$. Journal of Algorithms, 17(1):71-84, 1994.

[27] L. T. Ollmann. On the book thicknesses of various graphs. In F. Hoffman, R. B. Levow, and R. S. D. Thomas, editors, Southeastern Conference on Combinatorics, Graph Theory and Computing, volume VIII of Congressus Numerantium, page 459, 1973.

[28] S. V. Pemmaraju. Exploring the Powers of Stacks and Queues via Graph Layouts. $\mathrm{PhD}$ thesis, Virginia Polytechnic Institute and State University, U.S.A., 1992.

[29] V. R. Pratt. Computing permutations with double-ended queues, parallel stacks and parallel queues. In Symposium on Theory of Computing (STOC '73), pages 268-277, 1973.

[30] S. Rengarajan and C. E. Veni Madhavan. Stack and queue number of 2-trees. In D. Z. Du and M. Li, editors, Conference on Computing and Combinatorics (COCOON'95), volume 959 of $L N C S$, pages 203-212, 1995.

[31] F. Shahrokhi and W. Shi. On crossing sets, disjoint sets, and pagenumber. Journal of Algorithms, 34(1):40-53, 2000.

[32] R. E. Tarjan. Sorting using networks of queues and stacks. Journal of the ACM, 19(2):341-346, 1972.

[33] M. Yannakakis. Embedding planar graphs in four pages. Journal of Computer and System Sciences, 38(1):36-67, 1989. 